\title{
WestVirginiaUniversity
}

THE RESEARCH REPOSITORY @ WVU

Graduate Theses, Dissertations, and Problem Reports

2003

\section{Reinforcement and punishment during programmed instruction}

Stephen C. Scherer

West Virginia University

Follow this and additional works at: https://researchrepository.wvu.edu/etd

\section{Recommended Citation}

Scherer, Stephen C., "Reinforcement and punishment during programmed instruction" (2003). Graduate Theses, Dissertations, and Problem Reports. 2486.

https://researchrepository.wvu.edu/etd/2486

This Dissertation is protected by copyright and/or related rights. It has been brought to you by the The Research Repository @ WVU with permission from the rights-holder(s). You are free to use this Dissertation in any way that is permitted by the copyright and related rights legislation that applies to your use. For other uses you must obtain permission from the rights-holder(s) directly, unless additional rights are indicated by a Creative Commons license in the record and/ or on the work itself. This Dissertation has been accepted for inclusion in WVU Graduate Theses, Dissertations, and Problem Reports collection by an authorized administrator of The Research Repository @ WVU.

For more information, please contact researchrepository@mail.wvu.edu. 


\title{
REINFORCEMENT AND PUNISHMENT \\ DURING PROGRAMMED INSTRUCTION
}

By

Stephen C. Scherer

\author{
A DISSERTATION \\ Submitted to \\ The College of Human \\ Resources and Education \\ at \\ West Virginia University \\ in partial fulfillment of the requirements \\ for the degree of \\ Doctor of Education \\ in \\ Educational Psychology \\ Julie S. Vargas, Ph.D., Chair \\ Daniel Hursh, Ph.D. \\ Michael Perone, Ph.D. \\ Neal Shambaugh, Ph.D. \\ Richard Walls, Ph.D. \\ Department of Advanced \\ Educational Studies \\ Morgantown, West Virginia \\ 2003
}




\section{ABSTRACT \\ Reinforcement and Punishment During Programmed Instruction \\ Stephen C. Scherer}

Recommendations for improving answer accuracy during programmed instruction have included the use of punishment to decrease the rate of students' responding and to prevent careless mistakes. The effects of punishment during programmed instruction, however, have not been clearly identified. There is evidence that punishment increases attention and decreases response rates during learning tasks, but other effects on student responding, including escape from the learning task, have not been systematically examined. The present study was designed to systematically examine the effects of punishment as well as extinction during programmed instruction. Four college students were recruited to earn money while working on a program of instruction. A three-component MULT schedule was used to examine the effects of reinforcement, extinction, and punishment on students' answer accuracy, response topography, and frequency of escape behavior during learning. Conclusive results were not obtained from the analysis, however, as subjects failed to discriminate the different contingencies in effect during each of the components. The function of the loss of money for incorrect answers during programmed instruction could therefore not be determined in the present analysis.

Recommendations for procedural changes are provided that may allow future similar analyses to obtain reliable differences within response measures. 


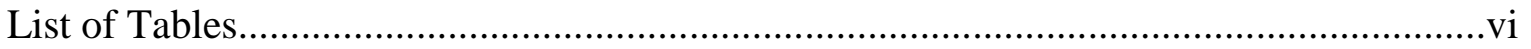

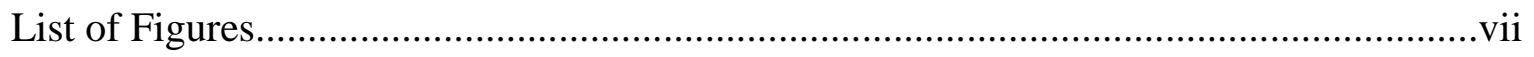

\section{Chapter 1}

Introduction

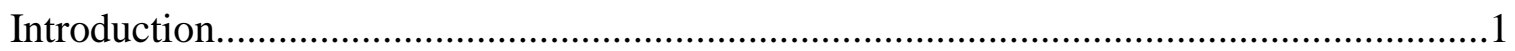

Programmed Instruction.....................................................................................

General Structure of Programs...................................................................2

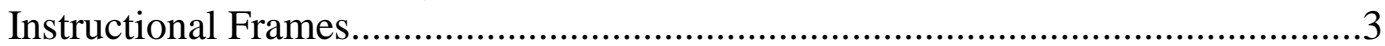

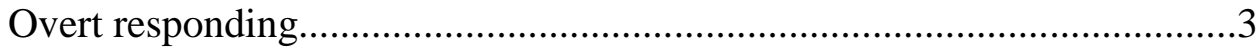

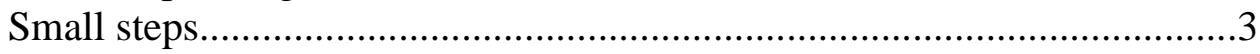

Immediate feedback.....................................................................4

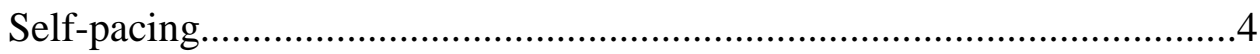

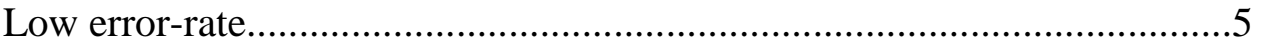

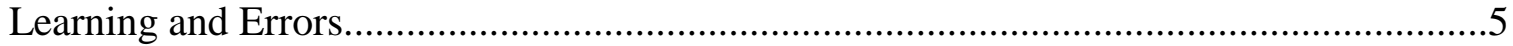

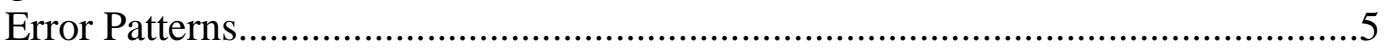

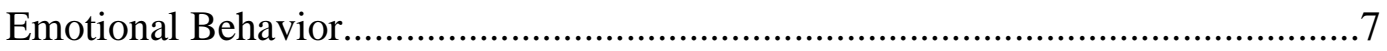

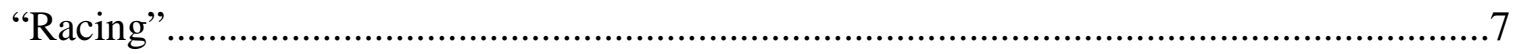

Chapter 2

Review of the Literature

Experimental Analyses of Punishment and Reinforcement During Learning.................10

Simple Discrimination Learning............................................................ 10

Punishment and Changes in Response Topography......................................12

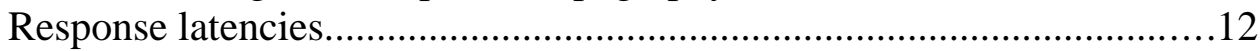

Orienting and observing responses..................................................13

Summary................................................................................... 15

Criticisms of Experimental Analyses of Punishment and Learning...............................15

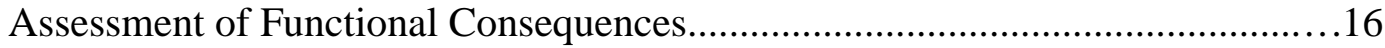

Stimulus Dimensions of Consequences........................................................17

Two-Choice Discrimination Tasks............................................................... 18

Group Experimental Designs..................................................................19

Limited Response Measures..................................................................20

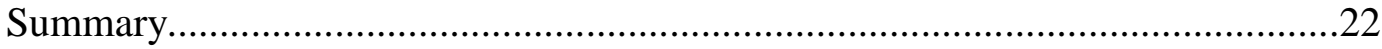

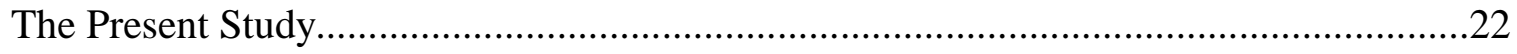


Chapter 3

Method

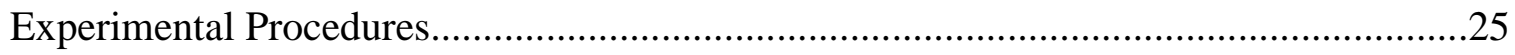

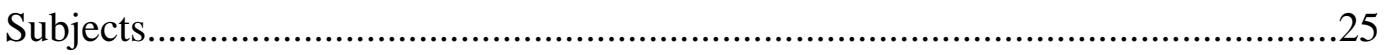

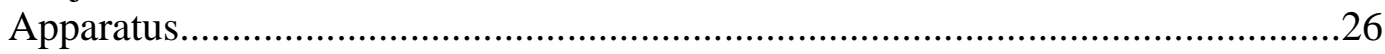

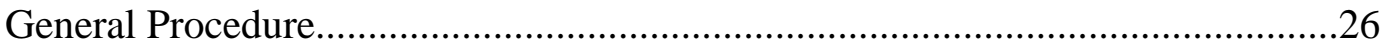

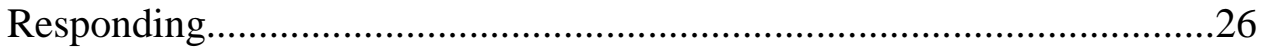

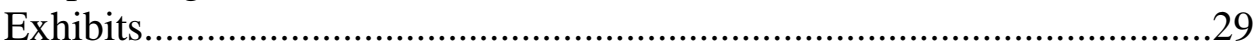

Frame status indicators............................................................... 30

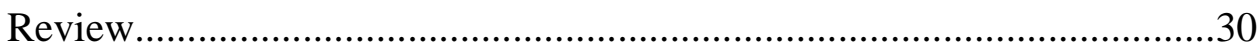

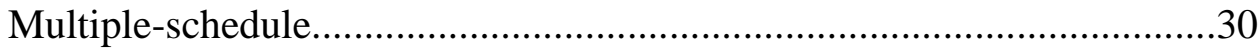

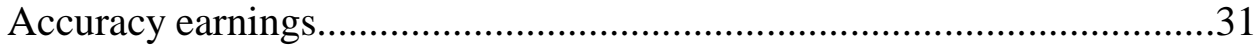

Component identification................................................................ 31

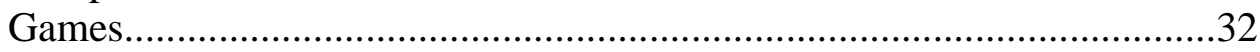

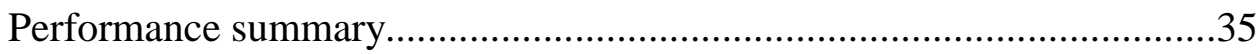

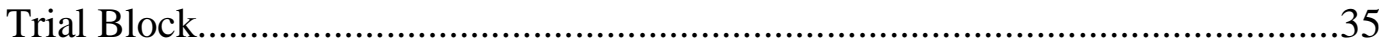

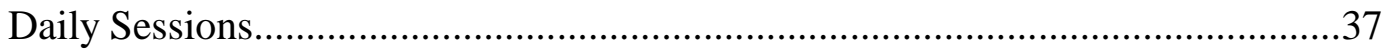

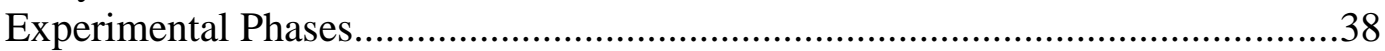

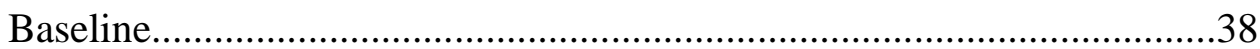

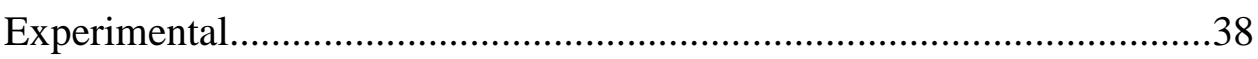

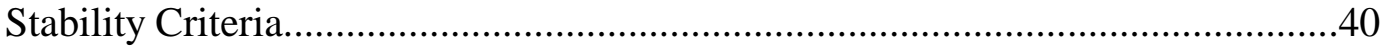

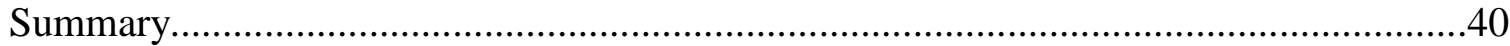

\section{Chapter 4 \\ Results}

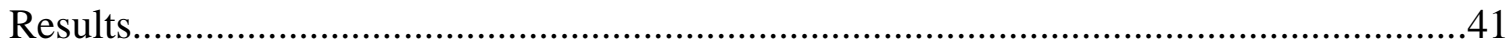

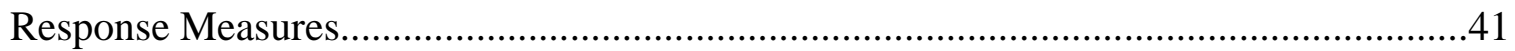

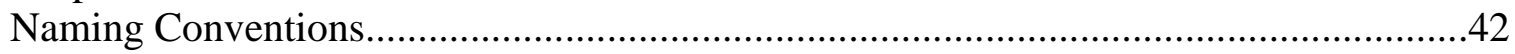

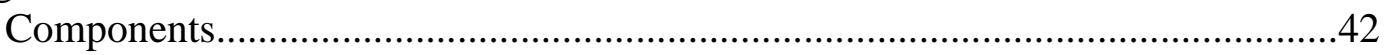

Baseline and Experimental Phases.............................................................42

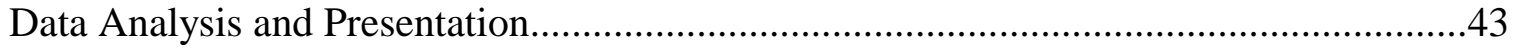

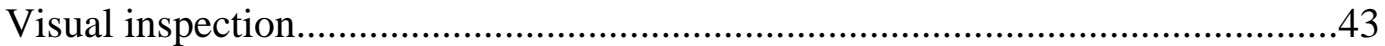

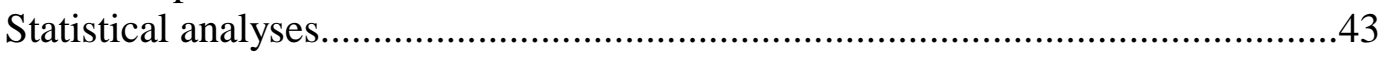

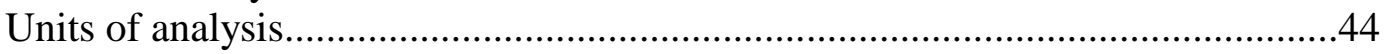

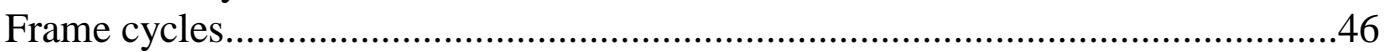

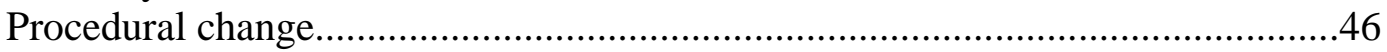

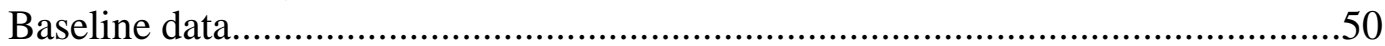

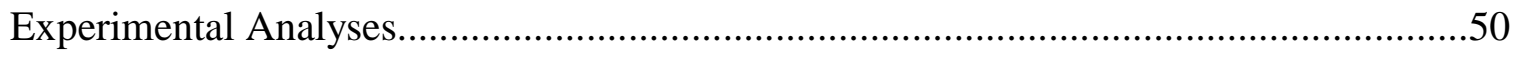

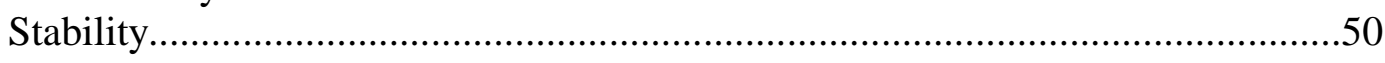

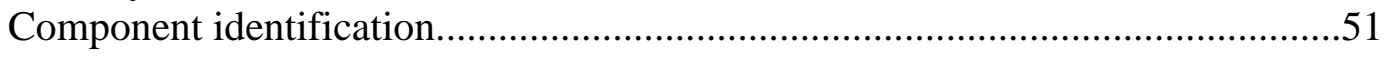

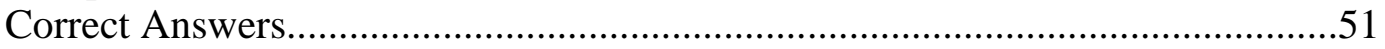

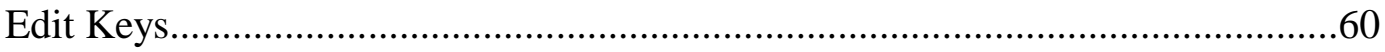

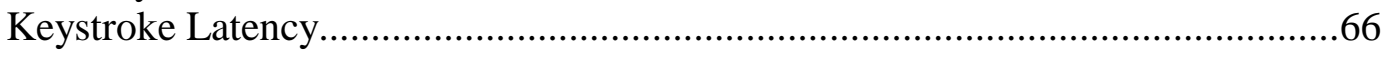


Viewing Correct Answers........................................................................66

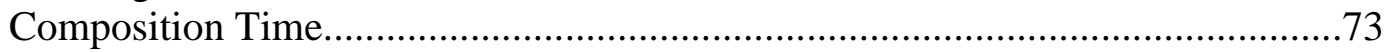

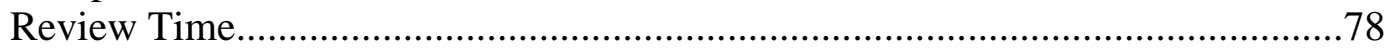

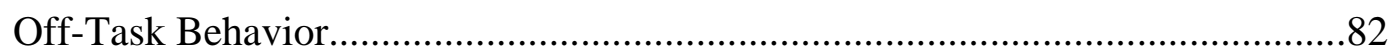

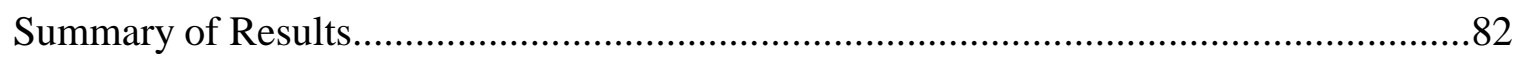

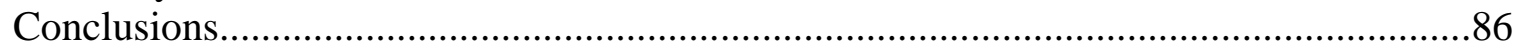

Chapter 5

Discussion

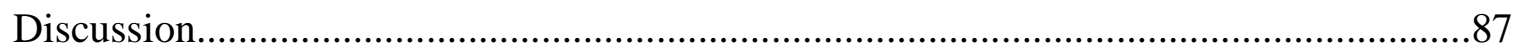

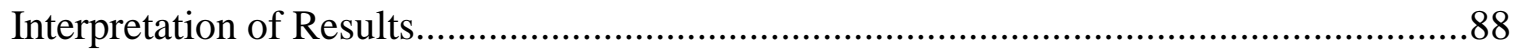

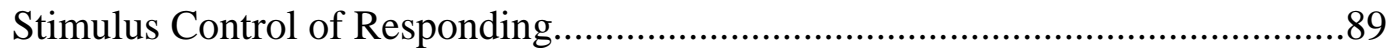

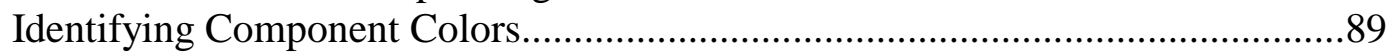

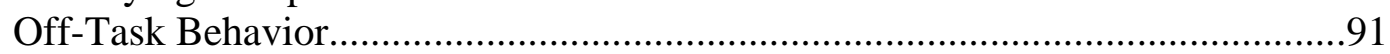

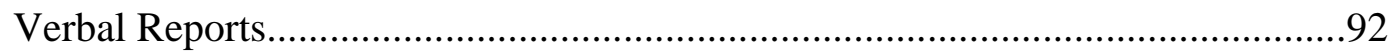

General Performance Decrements.................................................................92

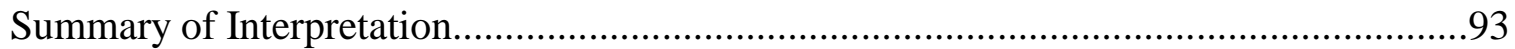

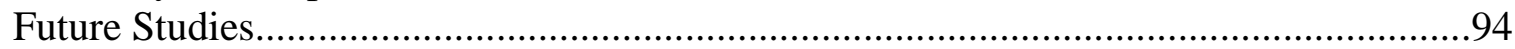

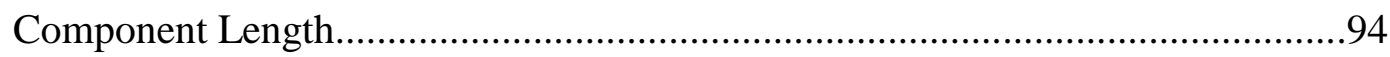

Number of Components........................................................................... 94

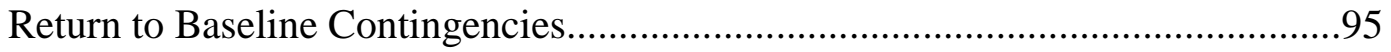

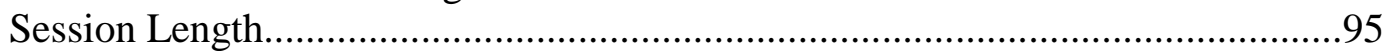

Subject Payments.................................................................................96

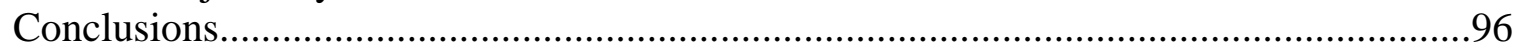

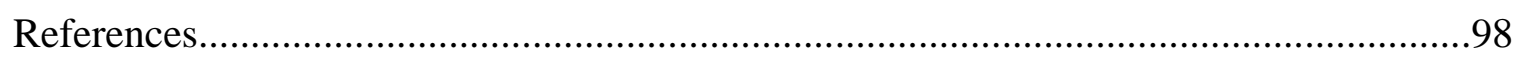

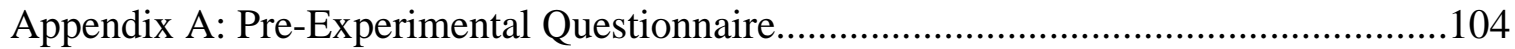

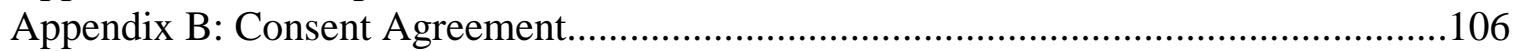

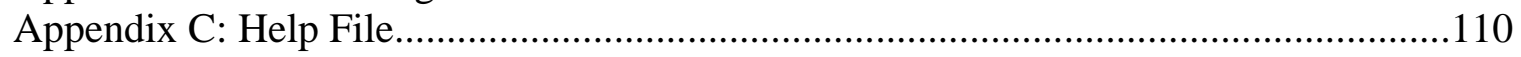




\section{List of Tables}

Table 1. Example multiple-schedule contingencies and correlated stimuli during experimental phases.

Table 2. Average earnings for all phases................................................................48

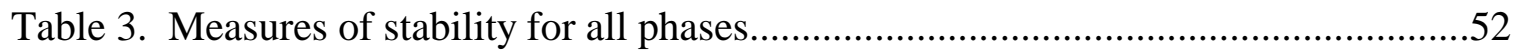

Table 4. Accuracy at selecting the stimulus correlated with the component in effect.

Table 5. Average percent correct across experimental phases.

Table 6. Statistical comparison of answer accuracy across experimental phases.

Table 7. Statistical comparison of the use of edit keys across experimental phases.

Table 8. Statistical comparison of latencies between key strokes. . .69

Table 9. Statistical comparison of time spent reviewing correct answers. . .72

Table 10. Statistical comparison of time spent composing answers.............................76

Table 11. Statistical comparison of time spent reviewing answers. .80 


\section{List of Figures}

Figure 1. Sample display screen (interface) for the program of instruction

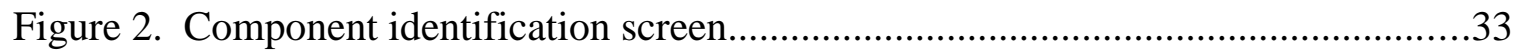

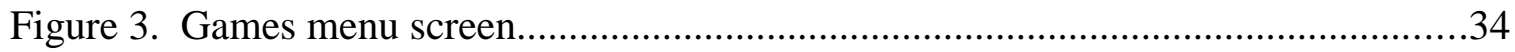

Figure 4. Earnings summary presented to the subject at the completion of each set.

Figure 5. Number of correct answers from bins of ten consecutive answers in each component.

Figure 6. Cumulative number of correct answers from bins of ten consecutive answers in each component.

Figure 7. Average number of correct answers for bins of ten consecutive answers in each component.

Figure 8. The number of edit keys (BACKSPACE and DELETE) used in each component when composing responses.

Figure 9. The average number of edit keys used for every ten consecutive answers in each component.

Figure 10. The sum of the average latencies between keystrokes used to compose individual answers

Figure 11. The average sum of the latencies between keystrokes used to compose individual answers

Figure 12. The sum of the time in seconds that subjects spent reviewing the correct answer when an answer was incorrect.

Figure 13. The average sum of the time in seconds that subjects spent reviewing the correct answer when an answer was incorrect

Figure 14. The sum of the time in seconds that subjects spent reading text and composing answers.

Figure 15. The average sums of the time in seconds that subjects spent reading text and composing answers. 
Figure 16. The sum of the time in seconds that subjects spent reviewing the status of their answers and correct answers when an answer was incorrect.

Figure 17. The average sums of the time in seconds that subjects spent reviewing the status of their answers and correct answers when an answer was incorrect

Figure 18. The sum of the time in seconds that subjects played video games. 


\section{Chapter 1}

Introduction

Programmed instruction is a technology of teaching based on empirically established principles of learning (Holland, 1960). The principles of learning upon which programmed instruction is based were established through intensive laboratory investigation of the conditions that reliably produced changes in the behavior of organisms, changes that are traditionally called learning. It is the systematic application of these empirically-based principles to the human learning process that accounts for programmed instruction's efficiency: students who learn with programs of instruction progress faster and retain what they have learned over longer periods than students who are taught with more traditional methods (cf. Kulik, Cohen, \& Ebeling, 1980). Of the numerous learning principles that account for programmed instruction's efficiency, one of the most commonly cited is that students should make a minimum of errors while they are learning. "Errorless" learning procedures (cf. Terrace, 1963) reduce the overall time and effort required by the student and avoid the disruptive effects errors on learning (e.g., Melaragno, 1960). Some students make numerous errors even when using well-designed programs of instruction, however. These students "race" through a program of instruction, responding rapidly and providing inaccurate answers (Crosbie \& Kelly, 1994; Kelly \& Crosbie, 1997; Munson \& Crosbie, 1998; Skinner \& Holland, 1965). A proposed solution to the problem of student "racing" is the use of mild punishment for incorrect responses, such as an imposed delay (Crosbie \& Kelly, 1994) or the loss of money (Munson \& Crosbie, 1998), and under some conditions these procedures appear to be effective deterrents to rapid and inaccurate responding. Some investigators, however, have reported the occurrence of undesirable side-effects of punishment during learning tasks, including increases in the rate of off-task behaviors and 
unusually high rates of errors when the punishment contingency is removed (Costello \& Ferrer, 1976; O'Leary \& Becker, 1969; Thomas, Becker, \& Armstrong, 1968).

Previous experimental analyses of response consequences during learning emphasized the reliable benefits of punishment for incorrect responses (e.g., Heron, 1978). There is some evidence that punishment during learning may produce undesirable effects as well, but these have been reported much less frequently (e.g., Costello \& Ferrer, 1976). It is possible that undesirable effects of punishment during learning are rarely reported because methodological limitations have precluded adequate assessment. The present study therefore attempted to replicate previous reports of both the desirable and undesirable effects of punishment during learning, especially during programmed instruction where effective procedures for eliminating rapid and inaccurate responding are needed.

\section{$\underline{\text { Programmed Instruction }}$}

\section{General Structure of Programs}

Programs of instruction are structured to allow students to progress at their own pace through a series of precisely ordered steps. Each of these steps is called an instructional frame. At each step, or frame, the student masters the material that is presented and is thereby prepared to take the next step in the progression. Instructional frames may involve reading and supplying missing text, solving a problem in mathematics, or even manipulating a physical model. For example, a popular program of instruction by Holland and Skinner (1961) contains nearly two thousand frames consisting of sentences with several missing words. The task of the student is to supply the missing text needed to complete the sentence(s). Each response is then evaluated before the next frame is presented. The evaluation of the accuracy of each response is typically conducted by the student who is presented with the correct answer for comparison. The student then 
proceeds to the next frame in the sequence.

$\underline{\text { Instructional Frames }}$

Many educational researchers have attempted to identify the essential characteristics of an instructional frame (e.g., Cook \& Mechner, 1962; Holland, 1960; Markle, 1967). Although disagreement exists regarding some aspects of instructional programming, most educational researchers are in agreement that certain principles of frame design are integral to the success of programmed instruction. It is these principles that account for programmed instruction's overall effectiveness.

Overt responding. Traditional educational practices emphasize various activities on the part of the learner, including listening to an instructor, reading instructional materials, or passively viewing instructional media. A limitation of these activities is that there is no assurance that the student is carefully reading, listening or otherwise attending to the task. One method of ensuring that the student is attending to an instructional task is to require the student to respond overtly to instructional materials. Overt responses can be required for trivial reasons, but programming the occurrence of overt responses is not a justification to "keep the student busy." Accurate responses made to well-designed programmed materials require careful examination and analysis of the instructional materials (Holland, 1961). This "attending" can then be immediately strengthened, or reinforced, by providing appropriate feedback to the student regarding the accuracy of each response.

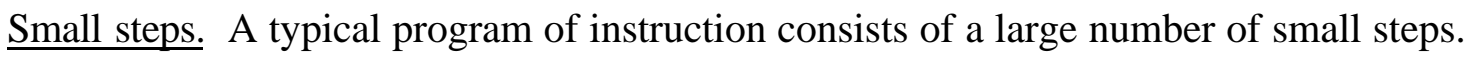
Within these small steps difficult concepts are slowly introduced and mastered or important points are reviewed. The effectiveness of a slow progression for introducing novel concepts and presenting material for review has been reliably demonstrated. Coulson and Silberman (1960) 
and Evans, Glaser and Homme (1962) examined student performance on two versions of a program of instruction. Frames that reviewed previously covered material or that functioned to transition from old to new material were removed from one version of the program (the "large step" program) while the other version of the program was left unmodified (the "small step" program). Superior posttest performances were reliably obtained for students using the "small step" program, demonstrating the importance of a gradual progression incorporating "redundant" materials and longer frame sequences.

Immediate feedback. Feedback is an essential component of programmed instruction. Each of the student's responses is normally evaluated immediately after composition and the results of the evaluation are presented to the student before he or she advances to the next instructional frame. The beneficial effects of feedback on student responding are robust and reliable, with students who receive immediate feedback performing more accurately during learning tasks and on posttest evaluations than students who don't receive immediate feedback (Angell, 1949; Anderson, Kulhavy, \& Andre, 1971; Kaess \& Zeamann, 1960; Meyer, 1960). These results support the characterization of feedback as reinforcement: feedback strengthens the response that it follows, especially the careful examination and response to instructional materials.

Self-pacing. Traditional classroom lectures, media presentations, and other instructional tools force all students to proceed at the same pace. Rapid learners are likely to become bored in such an environment while slower learners may be left behind. Programmed instruction makes accommodations for individual differences between students that are not otherwise possible in the traditional classroom environment. Each student interacts with a program of instruction presented by a teaching machine or computer. The machine operates much as an individual tutor would, allowing rapid or slow individual progress. In contrast to traditional educational 
methods, slow learners and fast learners can proceed at their own pace thus minimizing frustration and boredom (Skinner, 1986).

Low error-rate. A program of instruction produced with the above considerations taken into account will generate active responding on the part of the learner who is proceeding at his or her own pace through a series of finely graded steps. Records of student responding are easily generated in the process, and it is with these records that problematic areas in a program can be identified and corrected. Eventually the program can be refined to the point that students make very few errors, and ideally no errors at all, while using it.

An obvious advantage of allowing students to progress without errors is that there is a savings in time and effort when mistakes are avoided that would otherwise need to be corrected. The reasons to avoid the occurrence of errors are more than practical, however. By comparing the performances of students who learn with and without errors it has been possible to experimentally establish theoretical reasons for adopting "errorless learning" procedures (Terrace, 1963) with highly refined programs of instruction and other learning tasks. Experimental analyses have shown that the occurrence of errors may have complex and disruptive effects on the learning process that are difficult to counteract. The importance of "errorless learning" procedures with nonhuman organisms has been questioned by some researchers (esp. Kodera \& Rilling, 1976), but researchers examining human learning have provided strong evidence for minimizing the occurrence of errors.

\section{$\underline{\text { Learning and Errors }}$}

\section{$\underline{\text { Error Patterns }}$}

One means by which the occurrence of errors may interfere with progress on complex learning tasks is through the establishment of patterns of undesirable responding. This 
interference was demonstrated by Sidman and Stoddard (1967) and Stoddard and Sidman(1967) who used a program to train children to discriminate an ellipse form from circle forms. For the learning task, one ellipse and seven circles were projected onto an array of eight touch panels arranged in a square; reinforcers were contingent upon pressing the panel with the ellipse projected onto it. The task was initially easy, with the ellipse very flat and easy to discriminate relative to the array of circles. Progression through the task was made gradually more difficult by slightly increasing the circularity of the ellipse on successive trials. As the difficulty of the discrimination increased, the children began to make errors, selecting a circle instead of the slightly flattened ellipse. Often the children would get "stuck" and were unable to progress. The researchers carefully examined the errors made by children who were "stuck" in the program and found that the errors were systematic rather than random. Children who made errors tended to adopt "error-patterns," such as pressing the response panels in clockwise rotation on successive trials. These patterns were intermittently and accidentally reinforced by the occasional correct selection of the ellipse even though the child's responding was not under the control of the relevant stimulus dimension (i.e., degree of circularity). Each of the patterns adopted by the children was occasionally reinforced, though the patterns were incompatible with the development of stimulus control by the relevant property of the discrimination. Progression through the program was not only halted by allowing errors to occur, but was in fact inhibited, as the subjects responded in ways that had to be extinguished before progress could again be made (see also Kaess \& Zeaman, 1960).

"Error patterns" constitute a serious problem for a student using a programmed sequence of instruction. If an instructional designer has not anticipated the occurrence and establishment of such patterns, it is likely that progress through a linear sequence will be retarded until a special 
intervention is implemented that allows the student to "unlearn" the problematic responses. $\underline{\text { Emotional Behavior }}$

The occurrence of errors may also generate emotional responses, a problem that has been identified with both human and non-human learners (Holland, 1960; Schusterman, 1966; Terrace, 1963). Emotional responses are an undesirable side-effect of errors because strong emotions typically interfere with control by instructional stimuli. Disruptive emotional responses to errors are especially likely to occur if they occur consecutively (i.e., if there is a high "density" of errors) rather than if the errors are dispersed throughout the learning task (Kodera \& Rilling, 1976; Melangaro, 1960). The intensity of the resulting emotional response may not be trivial. Holland (1960) noted that "...students stop work when the material is so difficult that they make many errors. Furthermore, they become irritated, almost aggressive, when errors are made" (p. 219).

\section{"Racing"}

The occurrence of errors in a precisely defined learning sequence may generate responding that significantly interferes with the student's progress. Students may produce large numbers of errors even while using programs that are designed to produce "errorless learning," however. For example, students may be highly motivated to view the correct answer to a frame and therefore compose any response for evaluation (e.g., typing "garbage" letters). This practice is reinforced by the immediate appearance of the correct response and is especially likely to occur on difficult frames. Other students may respond similarly to escape from the learning task; these students respond rapidly and inaccurately to finish quickly.

Rapid and inaccurate responding maintained by escape from the instructional task or access to the correct answer has been called "racing" (Crosbie \& Kelly, 1994; Munson \& Crosbie, 
1998). The problem of racing and some possible solutions were described by Skinner and Holland (1965), who used a teaching machine with a program of instruction for college students. Their teaching machine was a wooden box with a lid into which the student placed a program disk. The program disk was divided into pie-shaped wedges, and on each wedge was a frame of instructional material that was visible through a window on the machine when the lid was down. Students wrote their responses to the frames on a strip of paper that was visible through a second window. When the frame was completed, the students lifted a lever on the front of the machine and moved the written response under a transparent cover; the correct response then appeared in the remaining portion of the frame window. If the student's response corresponded with the correct response, the student was to score the response as correct by moving the lever up and over which punched a hole in the response strip. This movement also altered the program so that the frame wouldn't appear again when the student worked around the disk a second time. This process was repeated until the student made a correct response to each frame. Throughout the process, authors noted the following:

There was a strong tendency to put down a quick, careless response in order to uncover the correct response in the machine. The more motivated the student, the stronger the tendency appeared to be. Many students were content to get the response right the second time around. It seems to be necessary to make correct first-cycle responses more important. This can be done by adding a slight punishment for a wrong response - for example, there could be fivesecond delay in moving on to the next frame when a response has been judged incorrect. Or we could appeal to the punitive techniques of educational institutions and keep a record of the number of first-cycle mistakes.... Another possibility would be to set up a "par" score for each tape and to require that the student who fails to complete the tape at or under par on the 
first cycle open the machine and start again - this operation supplying him with a fresh response strip and requiring him to answer all items again on the first cycle before proceeding to the second. (pp. 160-161)

Skinner and Holland's (1965) recommended solutions for the problem of "racing" involve the use of mild punishment for incorrect responses as a means of increasing the "importance" of constructing correct responses on the first attempt, and other researchers have recommended similar procedures (Crosbie \& Kelly, 1997; Munson \& Crosbie, 1998). Other researchers have found that the effects of punishment on a learner's behavior are complex and possibly detrimental, however (e.g., Costello \& Ferrer, 1976), and therefore the use of punishment during learning is not universally advocated. Prior to recommending the use of mild punishment during programmed instruction previously identified benefits and costs of punishment during learning will be examined. The present study will then be presented as an attempt to address perceived deficits in the existing body of research that deals with the complexities of punishment during learning. 


\section{Chapter 2}

Review of the Literature

\section{Experimental Analyses of Punishment and Reinforcement During Learning}

Experimental analyses of the effects of various reinforcement and punishment procedures during complex and simple learning tasks have been conducted with children and adult learners. Experimenters have examined: (1) reinforcement for correct responses, (2) punishment for incorrect responses, or (3) a combination of reinforcement for correct responses and punishment for incorrect responses. Of interest has been the differential effectiveness of reinforcement and punishment on reducing errors and facilitating learning.

\section{Simple Discrimination Learning}

A commonly used procedure to examine the effects of reinforcement and punishment on learning has been to examine learner performance on simple discrimination tasks. An example of a simple discrimination task was implemented by Penney and Lupton(1961). In their study, 45 elementary school children were given a two-alternative discrimination problem. The discriminative stimuli were two black squares. A diagonal was drawn from corner to corner on the face of each square, and a piece of white plastic was glued to the area above the diagonal. The face of the squares thus formed a black and a white right triangle. During the learning task, the child was seated in front of the experimental apparatus in which both of the squares were placed over wells that could hold pieces of candy. The apparatus was housed in a circular table that could be spun on its axis (i.e., a "Lazy-Susan") so that the squares could be shifted either in front of the experimenter or the child. On every trial the experimenter placed a piece of candy underneath one of the two squares and then spun the table around to the child (a vertical board centered in the table prevented the subject from observing the actions of the experimenter). The 
task of the child was to select the correct block by removing it from its housing; the child was allowed to select one block per trial. The experimenters arbitrarily determined that the correct response was the selection of the square for which the base of the white triangle was down (and under which the experimenter had placed a piece of candy). Control of the children's selection responses by the relevant property of the discrimination (the position of the white triangle) was established under three experimental conditions. For one group of students, jelly-beans (reinforcers) were available underneath the correct stimulus, and there was no consequence for an incorrect response. For another group of students, a loud tone (punisher) was administered following the selection of the incorrect stimulus, and there was no consequence for a correct response. A combination of reinforcement and punishment was used with a third group of students: the selection of the correct stimulus produced a jelly-bean, and selecting the incorrect stimulus produced a loud tone. The mean number of correct responses was compared between the three experimental conditions across five blocks of twelve trials (for a total of sixty trials). Individual $t$ tests revealed that there were no significant differences in performance between the three groups during the first block of trials. By the fourth block of trials, however, the performance of the punishment group was significantly better than the reinforcement group, $\underline{t}(28)=2.51$, but the performance of the combined group was not significantly better than the reinforcement group, $\underline{\mathrm{t}}(28)=.51$. By the last block of trials, the performance of the combined group was significantly better than the reinforcement group, $\underline{\mathrm{t}}(28)=2.56$, but not significantly different from the punishment group, $\underline{\mathrm{t}}(28)=.80$. The authors concluded that punishment alone was the most effective consequence for establishing the discrimination, and reinforcement alone was the least effective consequence.

Results similar to Penney and Lupton's (1961) were obtained by other researchers using the 
same or a similar two-choice discrimination procedure. During learning tasks, complex stimuli were often used that varied across a number of possibly relevant dimensions, including color, shape, position, and size. Establishing discriminative control of a subject's selection by isolated properties of the stimuli was invariably most difficult to accomplish using reinforcement alone; punishment in isolation or in combination with reinforcement was the most effective consequence for bringing the choice behavior of human subjects under the control of the relevant properties of the learning task (Brackbill \& O'Hara, 1958; Donahue \& Ratliff, 1976; Harris \& Tramontana, 1973; Hemry, 1973; Miller, Moffat, Cotter, \& Ochocki, 1973; Mueller \& Gumina, 1972; Ochocki, Cotter, \& Miller, 1974; Penney, 1967; Ratliff \& Root, 1974; Stevenson, Weir, \& Zigler, 1959; Tindall \& Ratliff, 1974; Trent, 1983; Witte \& Johnson, 1973). These results were replicated across special populations of children, including hyperactive boys (Cunningham \& Knights, 1978), impulsive children (Firestone \& Douglas, 1977), and delinquent boys (Moses, Ratliff, \& Ratliff, 1979). Facilitative effects of punishment relative to reinforcement on learning were also found during more complex tasks including mirror tracing (Borreson, 1973), reading (Brent \& Routh, 1978), and general educational activities (Foxx, 1984).

\section{Punishment and Changes in Response Topography}

Punishment of incorrect responses during learning is reliably accompanied by changes in the individual's response topography that may or may not be observed during reinforcement for correct responses. Analysis of these punishment-induced changes in response topography may reveal important mechanisms of action that are relevant to implementing punishment and reinforcement contingencies with programs of instruction.

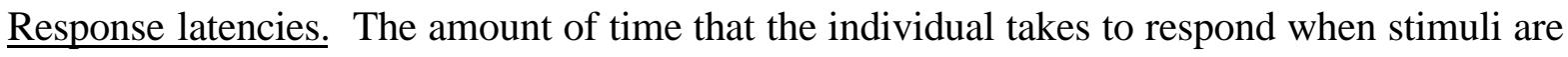
presented during a discrimination trial is called response latency. When punishment 
contingencies for incorrect responses are in effect, response latencies reliably increase (Brent \& Routh, 1978; Erickson, 1970; Hoge \& Stocking, 1912; Muenzinger, 1938; Munson \& Crosbie, 1998). For example, Erickson (1970) examined the performance of 48 college students on a two-choice discrimination procedure as a function of feedback in the form of punishment for the incorrect response. Subjects were required to discriminate between two rectangles of light that were positioned along a vertical axis, and the location of the stimuli along this axis was the relevant property of the discrimination task. For each trial, one of the rectangles was positioned "correctly" within plus or minus one inch of the arbitrarily selected target position. Selection of the "correct" rectangle produced no consequence, while selection of the "incorrect" rectangle produced one of three tones: soft $(72 \mathrm{~dB})$, medium $(84 \mathrm{~dB})$, or loud $(92 \mathrm{~dB})$. Subjects receiving the medium tone for incorrect responses reached a higher asymptote accuracy level $(\underline{\mathrm{M}}=.85)$ than subjects receiving either the soft $(\underline{\mathrm{M}}=.81)$ or loud $\underline{\mathrm{M}}=.77)$ tone as indicated by a significant quadratic main effect for sound, $\underline{\mathrm{F}}(1,24)=4.25, \mathrm{p}<.05$. Response latencies for subjects receiving the medium tone were also significantly higher $(\underline{\mathrm{M}}=1.33 \mathrm{~s})$ than subjects who received the soft $(\underline{\mathrm{M}}=1.04 \mathrm{~s})$ or loud tone $(\underline{\mathrm{M}}=1.29 \mathrm{~s})$; both linear and quadratic trends were significant, $\underline{F}(1,24)=7.77$ and 6.72 , respectively, ps $<.025$. The correlation between increases in response accuracy and increases in response latency suggests that effective punishment contingencies during learning may also reduce the rate at which responses are emitted.

Orienting and observing responses. During the interval between the presentation of an instructional stimulus and the selection or composition of an answer, the behavior of the student is "free to vary": the student may look away from the task, focus on a particular aspect of the instructional stimulus, or engage in other behavior. Response latencies provide crude measures of this intermediary behavior, but several researchers have specifically examined the topography 
of these responses as a function of reinforcement and punishment during learning. A commonly reported finding is that punishment contingencies produce increases in intermediary responses that may operationally define "attention," including orienting towards and looking at the task stimuli (Balaban, Rhodes, \& Neuringer, 1990; Fowler, Hochhauser, \& Wischner, 1981; Fowler, Spelt, \& Wischner, 1967; Muenzinger, 1938; Munson \& Crosbie, 1998; Ochocki et al., 1974; Penney, 1967; Stevenson et al.,1959; Taylor, 1974; Wischner, Fowler, \& Kushnick,1963; Wright \& Smothergill, 1967). For example, Penney (1967) systematically examined orienting responses during a learning task involving tactile discriminations. Subjects were 36 kindergarten children who were required to reach into a hole and consistently choose one of two blocks (either circular or a triangular) hidden behind a screen. Establishing discriminative control of responses by the shape of the squares was conducted under three conditions: reinforcement ( $M \& M$ candy), punishment (98 dB tone), and a combination of these two. Significant differences in the number of trials required to reach criteria were obtained between the groups, $\underline{F}(2,33)=3.87, \underline{p}<.05$. Individual $t$ tests indicated that children exposed to punishment alone required significantly fewer trials to reach criteria $(\underline{\mathrm{M}}=20.33, \underline{\mathrm{SD}}=10.03)$ than children exposed to the combination of candy and tone $\underline{\underline{\mathrm{M}}}=28.67, \underline{\mathrm{SD}}=7.54), \underline{\mathrm{t}}(10)=4.16, \underline{\mathrm{p}}<.01$. Additionally, children exposed to a combination of reinforcement and punishment required significantly fewer trials to reach criteria than children exposed to received only candy $(\underline{\mathrm{M}}=47.83, \underline{\mathrm{SD}}=10.87), \underline{\mathrm{t}}(10)=3.24, \underline{\mathrm{p}}<$ .01. The data from the original population were combined with the data from a second population of 18 kindergarten children for an analysis of the number of orienting responses (defined as individual touches to the concealed blocks) for each experimental group. Significant differences in the number of orienting responses were obtained between the groups, $\underline{F}(2,42)=$ $3.56, \underline{\mathrm{p}}<.05$. Individual $t$ tests revealed that the significant differences in the number of 
orienting responses were obtained between the punishment group $(\underline{M}=1.81)$ and the reinforcement group $(\underline{\mathrm{M}}=1.31), \underline{\mathrm{t}}(34)=2.07, \mathrm{p} 05$. Significant increases in number of orienting responses during punishment were therefore correlated with a significantly more rapid acquisition of the learning task. Punishment's generally facilitative effects on discrimination may be related to increases in the number of orienting or observing responses.

\section{$\underline{\text { Summary }}$}

Getsie, Langer, and Glass (1985) conducted a meta-analysis of the effects of reinforcement and punishment on human learning and concluded that reinforcement for correct responses alone is the least effective method for teaching discriminations. Punishment alone was found to be reliably more effective as feedback during discrimination training than reinforcement alone, and in many cases punishment alone was found to be superior to the combination of reinforcement and punishment; similar conclusions have been reached by investigators examining the effects of reinforcement and punishment on nonhuman learning. The facilitative effects of punishment on learning may be related to its effect of increasing the frequency of observing and orienting responses as measured by increases in response latencies. A review of the experimental literature examining the effects of reinforcement and punishment on learning therefore suggests that punishment for incorrect responses should be considered for reducing the rate and increasing the accuracy of student responding during programmed instruction.

\section{Criticisms of Experimental Analyses of Punishment and Learning}

The reliably superior effects of punishment relative to reinforcement during discrimination training that have been reported deserve closer examination. It is possible that the conclusions of researchers examining the effects of reinforcement and punishment on learning are of limited generality. Sources of limitations on the generality of previous conclusions include the use of 
problematic experimental procedures, the extensive reliance on group statistical comparisons, and the use of overly-simplified learning tasks and consequences.

\section{Assessment of Functional Consequences}

Several of the studies that compared the effects of punishment and reinforcement on children's learning provided consequences for response accuracy that were of questionable functional value. These consequences included items that could be exchanged for tangible reinforcers, including money (Brent \& Routh, 1978; Hemry, 1973) and tokens exchangeable for a toy (Cunningham \& Knights, 1978; Firestone \& Douglas, 1977; Tindall \& Ratliff, 1974). The experimental methods described in each of these studies do not indicate that an assessment of the reinforcing or punishing effectiveness of these consequences was conducted prior to examining their effects on learning. It is possible that tokens and money were not functional reinforcers for the children, or that they functioned only as very weak reinforcers.

A similar problem exists for studies examining reinforcement and punishment during children's learning where verbal praise and/or reprimands were provided as a consequence for response accuracy (Firestone \& Douglas, 1977; Foxx, 1984; Hemry, 1973; Moses et al., 1979; Miller et al., 1973; Mueller \& Gumina, 1972). The functional value of a consequence such as praise or reprimands may vary widely among individuals, as was demonstrated by Costello and Ferrer (1976). These researchers used a single-subject design to examine the effects of punishment and nonpunishment (extinction) of errors that were produced by six children during a program of articulation instruction. The program was designed to correct children's errors in pronouncing phonemes, and each subject earned tokens for correct pronunciations throughout all conditions of the experiment; the tokens were exchangeable for a toy. In addition to the continuous positive reinforcement for correct pronunciations, three different punishers were 
alternately used for incorrect pronunciations: 1) the word "No!"verbalized by the experimenter, 2) a loud tone, and 3) the withdrawal of a token. After a baseline period of four sessions, each of these punishers was applied individually for three daily one-half hour sessions before transition to three sessions of nonpunishment for incorrect pronunciations. After completion of three sessions of nonpunishment, the second punisher was applied for three sessions, followed by three sessions of nonpunishment, and the process was then repeated with the third punisher. The data were analyzed via visual inspection. An important observation was that the effectiveness of the punishers varied widely across subjects. None of the three purported punishers was functional for all of the subjects, but at least one of the punishers was functional for each subject. For example, verbal reprimands as punishers for incorrect responses during the program of articulation instruction reliably reduced errors in phoneme pronunciation for two or possibly three of the six subjects; for the other three subjects, verbal reprimands were functionally neutral or actually accelerated error rates. These results indicate that the function of verbal feedback may vary widely across individuals, and investigators may not be justified in comparing the performance of learners as a function of consequences (e.g., verbal reprimands) that are assumed a priori to have a given behavioral effect.

\section{$\underline{\text { Stimulus Dimensions of Consequences }}$}

Direct comparisons of the effects of reinforcement and punishment on learning are complicated by the use of reinforcers and punishers of different stimulus dimensions. This problem is illustrated by the use of a loud tone as a punisher of incorrect responses and administration of tokens as reinforcers of correct responses during discrimination training (e.g., Hemry, 1973; Ratliff \& Root, 1974; Tindall \& Ratliff, 1974). The effectiveness of tokens on the behavior of an individual presupposes an extended history of exposure to similar reinforcers that 
were accrued and exchanged for more tangible outcomes. Loud tones as punishers are likely to be effective as consequences irrespective of the individual's prior history of reinforcement. The finding that loud tones as punishers are reliably more effective at increasing response accuracy than are tokens as reinforcers may be therefore be attributed to functional differences between the consequences.

Differences in the dimensions of stimuli used as consequences during learning are also problematic when the administration of a reinforcer involves a consummatory response while the punishing stimulus doesn't require a consummatory response. For example, Penney (1967) and Penney and Lupton (1961) used candy as a reinforcer and intense noise as a punisher during learning. As several other researchers have noted (Donahue \& Ratliff, 1976; Hemry, 1973; Ratliff \& Root, 1974; Tindall \& Ratliff, 1974), the consummatory response required for the reinforcer (i.e., eating the candy) may involve responses that interfere with control by the relevant properties of the task. The absence of a similar consummatory response associated with the punishing stimulus meant that learners were less likely to be distracted by the punishing consequence. The differences in distractibility of the response consequences may account for the significantly higher rates of accuracy obtained during the punishment condition; unambiguous comparisons of the effects of reinforcement and punishment on learning are difficult or impossible under these circumstances.

$\underline{\text { Two-Choice Discrimination Tasks }}$

Researchers examining the effects of punishment and reinforcement on learning typically used a two-choice discrimination procedure (cf. Penney \& Lupton, 1961) to examine the effects of reinforcement and punishment on children and adult learning (Brackbill \& O'Hara, 1958; Cunningham \& Knights, 1978; Donohue \& Ratliff, 1976; Erickson, 1970; Harris \& Tramontana, 
1973; Miller et al., 1973; Moses et al., 1979; Mueller \& Gumina, 1972; Penney, 1961; Penney \& Lupton, 1967; Ratliff \& Root, 1974; Tindall \& Ratliff, 1974; Witte \& Johnson, 1973). The conclusion that punishment produces increases in correct responding during a two-alternative discrimination procedure could be an artifact of the experimental procedure: the punishment of one of two available responses necessitates an increase in the probability of the only alternative response (cf. Azrin \& Holz, 1966). Under these conditions, it is unknown to the researcher as to whether the subject has learned to engage in the correct response or only to avoid the incorrect response. This distinction may not be critical for performance on a two-choice discrimination procedure, but the distinction is relevant for learning tasks that require the student to construct a response rather than to select it. During programmed instruction, for example, punishment of an incorrect response will not by definition increase the probability of the correct response, as punishment does not tell the organism what to do but rather what not to do. Results of studies using two-choice discrimination procedures may therefore have only a limited generality to complex learning tasks such as programmed instruction or even less complex discrimination tasks involving three or more alternatives.

\section{Group-Experimental Designs}

The present review (see also Getsie et al., 1985) of the effects of punishment and reinforcement on learning is largely based on the results of studies that used statistical comparisons of between group differences (for exceptions in the present review see Costello \& Ferrer, 1976; Munson \& Crosbie, 1998). Comparisons of response accuracy were conducted between groups of individuals, and each individual within a group was exposed to one experimental condition (reinforcement, punishment, or a combination of reinforcement and punishment). Statistical analyses required the averaging of individual performances within each 
group for the necessary comparisons of within and between group variability. Conclusions based on group statistical comparisons of averaged performances were limited to statements about significant differences between the groups rather than statements about individual responding as a function of a reinforcement and punishment (cf. Sidman, 1960). Possibly relevant individual differences could not be detected with these experimental designs. This problem was highlighted by Costello and Ferrer (1976), who found wide individual variability in the effects of consequences at the level of the individual (see above). These differences would not have been identified if the authors had used statistical procedures that mathematically obscure betweensubject differences through the process of averaging performances.

\section{Limited Response Measures}

Statistical analyses may result in limited descriptions of the relations between independent and dependent variables at the level of the individual, and this problem may be compounded by the reliance on hypothetico-deductive experimental models. Researchers using this model may construct experiments that are designed to test a hypothesis while contributing little to related areas of inquiry. Michael (1974) criticized the use of a hypothetico-deductive model with individual organism research, noting that "[t]he results from such experiments are... less useful for any purpose than answering the specific question being asked in that experiment." In the present review, for example, many experiments were designed to assess the differential effects of reinforcement and punishment on a single dependent measure: trials to criterion or the average number of correct responses (Borreson, 1973; Brackbill \& O’Hara, 1958; Donahue \& Ratliff, 1976; Fago \& Fowler, 1972; Firestone \& Douglas, 1977; Fox, 1984; Fowler et al., 1967; Harris \& Tramontana, 1973; Hemry, 1973; Hoge \& Stocking, 1912; Miller et al., 1973; Moses et al., 1979; Mueller \& Gumina, 1972; Muenzinger, 1934; Penney, 1961; Penney \& Lupton, 1967; 
Ratliff \& Root, 1974; Reuter \& Chung, 1988; Stevenson et al., 1959; Tindall \& Ratliff, 1974; Warden \& Aylesworth, 1927; Wischner et al., 1963). While these studies addressed the hypotheses of the experimenters regarding reinforcement and punishment during learning, they failed to address one of the most important criticisms of the use of punishment, namely its undesirable effects on unpunished responding (cf. Newsom, Favell, \& Rincover, 1983; Perone, 1990). Examples of relevant side effects of punishment in clinical settings include increases in emotional behavior (Merbaum, 1973), aggression (Carr, Newsom, \& Binkoff, 1980), and escape or avoidance (Azrin \& Holz, 1966). During learning, a relevant side effect that may result from the use of punishment is an increase in off-task or disruptive behaviors. For example, Costello and Ferrer (1976) measured the number of off-task behaviors as well as the percent of incorrect phoneme articulations per session as a function of the presence or absence of one of three alternating punishment contingencies (see above). Off-task behaviors were defined as vocal and motor responses that were incompatible with providing the correct answer to the programmed stimulus (e.g., banging a fist on the table, playing with tokens, or making animal sounds). Each of the response measures in the study was shown to systematically vary as a function of the presence or absence of functional punishment contingencies for incorrect phoneme articulations. When a functional punisher was applied, decreases in both the percent of incorrect responses and the number of off-task behaviors were obtained. Unexpected results were obtained when the punishment contingencies were removed. During the subsequent nonpunishment conditions, the authors noted the occurrence of off-task behavior "highpoints," described as "data points that show an especially high rate of off-task behaviors in comparison to the points surrounding them within or across experimental conditions" (p. 56). The percent of incorrect phoneme articulations was similarly affected by the presence or absence of punishment contingencies: 
transition to a nonpunishment condition was often accompanied by unusually high percentages of errors in pronunciation. The increases in off-task behavior and error rates that were observed when the punishment contingency was removed were important side-effects of punishment that could not have been detected if the experimenters had relied only on a single response measure, e.g., percent of correct responses, to evaluate the overall effectiveness of the punishment contingencies.

$\underline{\text { Summary }}$

General conclusions about the effectiveness of reinforcement and punishment during learning are presently limited by the extensive use of experimental designs that preclude a systematic analysis of individual responding. However, the evidence that punishment can be beneficial to responding during programmed instruction is suggestive. A more thorough analysis of punishment's effects is needed, and this analysis should involve parametric manipulations of punishing stimuli during programmed instruction with continuous measures of student responding and within-subject replications (e.g., Costello \& Ferrer, 1976; Munson \& Crosbie, 1998).

\section{The Present Study}

The present study was designed to address some of the deficiencies in the existing experimental literature on the use of punishment and reinforcement during learning, and specifically during programmed instruction. A parametric manipulation of punishment contingencies was conducted to assess the effects of punishment on answer accuracy, response topography, and escape behavior. Several controls were implemented in an attempt to improve upon previous analyses in this area. First, the reinforcing and punishing stimuli remained in the same stimulus dimension in the form of money. As Skinner (1953) noted, money is an ideal 
consequence for several reasons. Money is easy to quantify and present to the subject; for example, numerical counters can be incremented (reinforcement) or decremented (punishment through response-cost) contingent upon each response. Second, monetary values of the reinforcing and punishing consequences can be easily manipulated, thus allowing for parametric manipulations of the reinforcer and punisher magnitudes. Finally, unlike consequences such as food or toys, money is generally effective irrespective of the momentary condition (i.e., level of deprivation) of the student.

For the present study, responding was first maintained with reinforcement alone to establish a characteristic pattern of responding, or a response baseline, against which the effects of punishment were evaluated. Punishment contingencies were then applied concurrently with the reinforcement contingencies in place. This procedure was accomplished through the use of a three-component multiple (MULT) schedule of reinforcement. A MULT schedule is defined as a schedule that consists of two or more schedules (components) that alternate, and each schedule is accompanied by a unique stimulus for as long as the schedule is in effect (Skinner \& Ferster, 1957). Initially, accurate responding was reinforced in each component of the MULT schedule. After responding stabilized, a punishment contingency was introduced into one of the three components. An extinction contingency, where no money was earned for a correct response if the first attempt was incorrect, was introduced in the final component. The effects of the punishment and extinction contingencies were then assessed both within and between components.

An attempt was made to control the conditions (hereafter referred to as phases) of the experiment to such a degree that any changes in responding that occurred when the punishment and extinction contingencies were implemented could be reliably attributed to those 
contingencies alone. To obtain this degree of control, important features of the method for this experiment were adapted from Madden (1995) and Madden and Perone (1999). These researchers examined the sensitivity of human subjects to schedules of reinforcement that were concurrently available, i.e., subjects could choose which schedule would be in effect at a given time. Subjects reliably chose the "best" schedules when they were required to identify the stimulus correlated with each schedule, suggesting that this requirement facilitates control by component contingencies. A similar procedure was used in the present study, where at randomly chosen times subjects were asked to identify the component currently in effect. 


\section{Chapter 3}

\section{Method}

\section{$\underline{\text { Experimental Procedures }}$}

\section{$\underline{\text { Subjects }}$}

Three males and one female participated in the experiment. Subjects were from 24 to 29 years of age and were full-time students at West Virginia University. The subjects were recruited with advertisements posted at the West Virginia University campus student job board, advertisements posted within the College of Human Resources and Education, and through visitations to classrooms. Potential subjects were screened using a questionnaire prepared by Madden (1995; Appendix A). Individuals who indicated a need for money, no previous experience with psychological experiments, and minimal exposure to the principles of behavior analysis were paid $\$ 5.00$ to complete a trial session (see below) to determine if they could successfully work on the task. Three potential subjects, labeled S01, S03, and S06, completed only the trial session. Subjects S01 and S03 indicated that they were not interested in working further on the project and their participation was discontinued. Subject S06 was a non-native speaker of English and her performance on the trial session was inadequate: by mutual agreement the decision was made for her to not participate. Those subjects who completed the trial session with adequate performance and who expressed interest in continuing with the project (subjects S02, S04, S05, and S07) were asked to read and sign an informed consent form (Appendix B) before proceeding further. Three of theses subjects (S02, S05, and S07) were males between the ages of 24 to 27 who were studying engineering at the graduate level. Subject S05 was a non-native speaker of English. Subject S04 was a 29 year old female graduate student in the College of Human Resources and Education. She was also a non-native speaker of 
English.

\section{$\underline{\text { Apparatus }}$}

Sessions were conducted in a $10 \times 8$ foot room containing a desk, chair, a three-ring binder, and an IBM PC-compatible computer. A printer was located to the left of the table on the floor and was not used during the experiment. Subjects sat at the desk and used the computer to complete the task. The computer had a 17-inch VGA color monitor, two portable speakers, a mouse, a keyboard, and headphones that plugged into the computer's audio system. The computer speakers were connected to a portable radio that served as a white noise source throughout the session. The three-ring binder contained exhibits for the program and a help document (described below).

\section{$\underline{\text { General Procedure }}$}

Daily sessions lasted for approximately one hour, during which time the subject worked to complete at least one set of instructional frames from a program of instruction (Holland \& Skinner, 1961). The original program of instruction consisted of 53 sets with an average of 37 frames per set. Four of the sets in the original program were designed to review previously covered material and were excluded from the present study (sets 17, 29, 41, and 53). The first set in the program was used for trial purposes (described below) and subjects' performance on this set was not included in the present analysis. The interface for the program is shown in Figure 1.

$\underline{\text { Responding. }}$ Text and graphics for each instructional frame were presented to the subject who then typed answers to the frame in numbered text-entry blanks. Each frame required from one to five responses. Active text-entry blanks were colored light grey and inactive text-entry blanks were colored dark grey. Active text-entry blanks were colored blue if the cursor was 


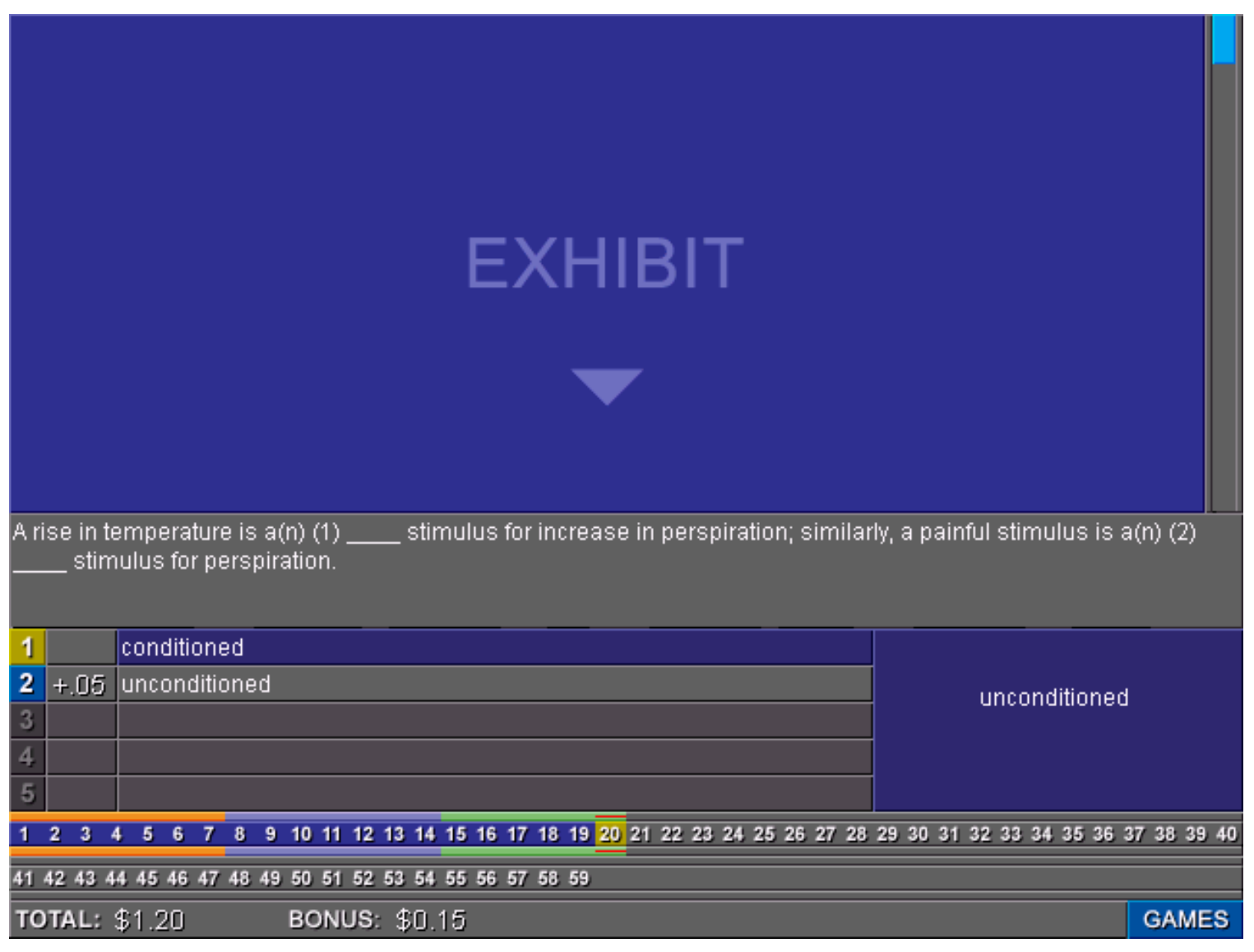

Figure 1. Sample display screen (interface) for the program of instruction. The top portion of the interface was reserved for the display of exhibits. Frame text appeared near the middle of screen. Answers were typed in the blanks directly below the frame text and were evaluated by pressing the ENTER key on the keyboard. The number next to the subject's answer turned blue if the answer was correct and yellow if incorrect. Correct answers were revealed by pressing the number corresponding to the answer on the keyboard (the number 1 is being held down in the figure). Frame numbers (the numbers 1-59 in this set) indicated the status of each frame (grey = not completed, blue $=$ completed and correct, yellow $=$ completed with at least one incorrect answer, red = current frame). Components of the MULT schedule were indicated by thin bands of color bounding the top and bottom of each numbered indicator (here frames 1-20). Earnings 
were located at the bottom of the screen. Games could be accessed at any time by clicking the GAMES button located at the bottom right corner of the interface. 
within the blank. Subjects could navigate among the active text-entry blanks by using the UP ARROW, DOWN ARROW, TAB, or SHIFT + TAB key combination. Pressing the RETURN key placed the cursor into the first empty text-entry blank if at least one text-entry blank was empty or contained only spaces. Pressing the RETURN key after answers were composed in each active text-entry blank initiated the evaluation sequence and disabled keyboard input into the text-entry blanks.

A programmed pattern-matching routine was used to determine the accuracy of each answer during the evaluation sequence. Numbered indicators next to each answer changed from lightgrey to blue if the answer was correct and yellow if the answer was incorrect. The frame number indicator at the lower portion of the interface changed from light grey to blue if all of the answers for the frame were correct and yellow if at least one of the answers was incorrect. The correct answer could be revealed by pressing the corresponding number key on the keyboard. When the number key was pressed the color of the text-entry blank and the panel to the right of the blank changed from light-grey to blue and the correct answer was displayed on the panel. The textentry blank and panel changed from blue to light-grey and the correct answer was erased when the number key was released. Subjects could then advance to the next frame in the set by pressing the SPACEBAR. Keyboard events were then enabled and the next frame was reinstated.

Exhibits. Supporting materials, including text and graphics, were contained in exhibits that accompanied many of the program sets. Exhibits were converted into images and displayed at the top portion of the interface. A button to the left of the exhibit allowed the subject to scroll the exhibit up or down to facilitate viewing. Exhibits were also photocopied and placed in a binder that was available throughout the experiment. 
Frame status indicators. A sequence of numbers corresponding to the frames of the current set were displayed in parallel horizontal rows across the bottom of the program screen. Frame one of a set was represented by the number " 1 ," etc. The background color of each of the numbers indicated the status of the corresponding frame. A light-grey background indicated that the subject had not yet advanced to that frame. A red background color indicated that the corresponding frame was currently active. A blue background indicated that the answers provided for the corresponding frame were correct. A yellow background indicated that at least one of the answers provided for the corresponding frame was incorrect.

Review. A review sequence was begun following completion of all of the frames in a set. All frames with at least one incorrect answer were presented again, beginning with the first frame containing an incorrect item and ending with the last frame. A frame was only completed and dropped from the review sequence when all of the answers to that frame were correct. If a frame presented for review contained multiple answers, those answers that were previously correct were displayed in their corresponding text-entry blanks and could not be edited. Thus, only responses that had been missed had to be redone. The review sequence was repeated until all frames had been correctly answered.

Multiple schedule. A three-component MULT schedule was in effect throughout each set. Each component of the MULT schedule was active for 6 minutes and was indicated by the color (green, purple, or orange) of the area immediately above and below each frame number (see Figure 1). When the subject advanced to a new frame the color of the component indicator changed from light-grey to the color of the component in effect. For example, if the MULT schedule component was indicated by the color orange, the color of the area immediately above and below the frame number changed to orange as subject advanced to the first frame in that 
component.

Component changes occurred only when the subject advanced to a new frame and the component timer had expired. For example, if the component was orange and the timer for the component had expired, the color of the component changed to purple instead of orange when the subject advanced to the next frame. Component contingencies and schedule-correlated stimuli changed only during the first cycle through a set and remained in effect during the review sequence. Therefore the component that was in effect when the subject first answered a particular frame was reinstated for that frame when another attempt was needed to provide a correct answer.

Components always occurred in the following order: green -> orange -> purple. The first component of each set was randomly predetermined with the exception that the same component could not be the first component for more than two consecutive sets. The initial component for each set was the same for all subjects.

Accuracy earnings. Subjects earned money by providing correct responses. If a response was correct, the amount of money that was earned for that response was displayed immediately to the left of the answer (e.g., "+.07”; see Figure 1). All correct responses during the first-cycle through a set earned money. Correct responses during the review sequence were reinforced with money depending on the component schedule in effect and the experimental phase. The amount of money earned for correct responses was added to a running total when the subject advanced to a new frame. This total was displayed in the lower left corner of the interface next to the TOTAL label. The total amount earned for each set was recorded at the completion of the set and the running total was initialized to $\$ 0.00$ when a new set was begun.

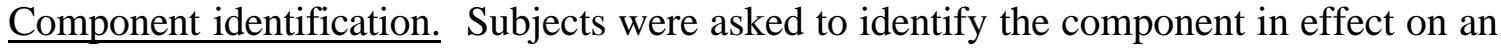


average of every seventh frame in a set. If a frame was targeted for component identification, pressing the ENTER key after composing answers for a frame hid the program interface and displayed the component selection screen (see Figure 2). The component selection screen consisted of three colored squares (green, orange, and purple) below the words "Select the current component color with your mouse." The subject then used the computer mouse to select the colored square that corresponded to the color of the component in effect for the current frame. A white outline appeared around a colored square when the subject placed the mouse over it, and the colored square was selected by clicking on it with the left mouse-button. If the color of the square selected by the subject corresponded to the component color of the current frame, the colored squares were erased and the message "Correct. $\$ 0.15$ has been added to your bonus earnings." replaced the original message. If the subject selected the incorrect colored square, the message "Incorrect. No money has been added to your bonus earnings." appeared. A button labeled CONTINUE was displayed below the feedback message and returned the subject to the program when clicked. Bonus earnings were updated with every correct selection and the value of the bonus earnings was continually displayed at the bottom of the program interface next to the BONUS label (see Figure 1). The total amount of bonus earnings for each set was saved at the completion of the set and the running total was initialized to $\$ 0.00$ when a new set was begun. All timers were suspended while the component identification procedure was in effect.

Games. At any time during a set the subject could access video games by pressing the blue GAMES button located in the lower right corner of the program interface. When the button was pressed, the program interface was replaced with a menu of buttons (see Figure 3). Labels on each button indicated the game that could be accessed by clicking on it. Pressing the DELETE 


\section{Select the current phase color with your mouse.}
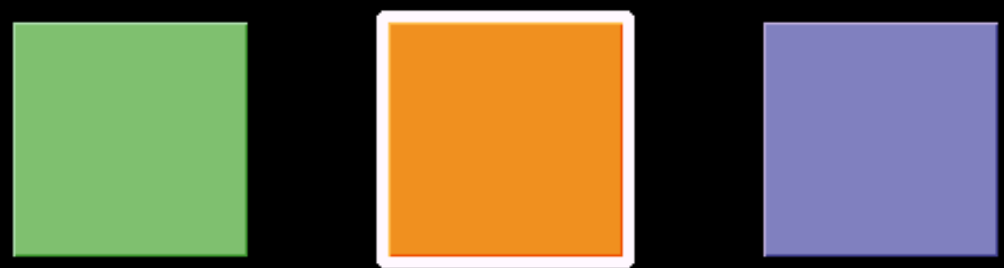

Figure 2. Component identification screen. The subject's mouse cursor (not visible) is currently over the orange (middle) square. 


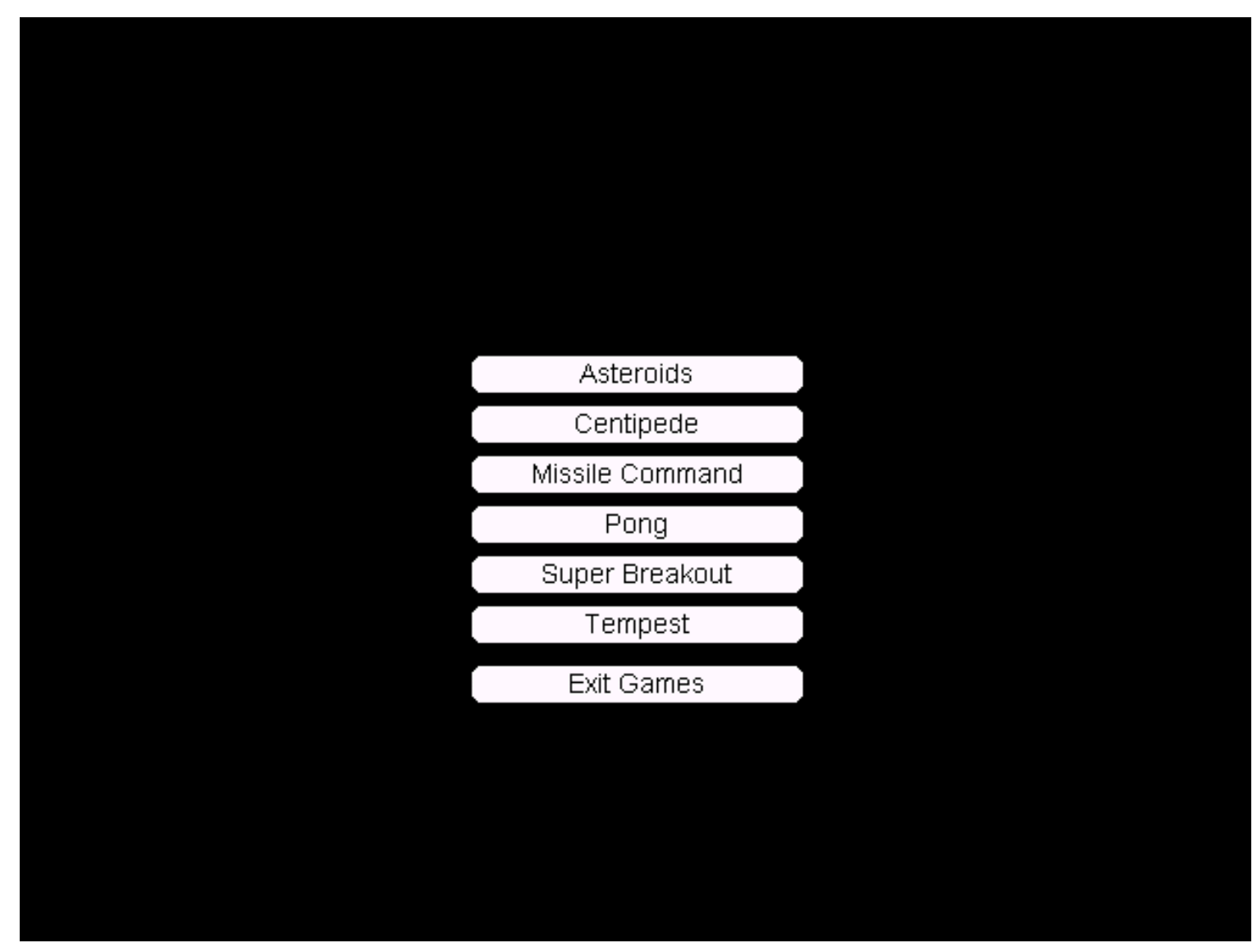

Figure 3. Games menu screen. Clicking on any of the buttons opened a computer game, and clicking on the EXIT GAMES button cleared the menu and returned the subject to the program. 
key while playing a game quit the game that was being played and returned the subject to the games menu. Audio for the games was delivered through the headphones that the subject wore throughout the session. Session timers were suspended when the GAMES button was clicked and were resumed when the subject clicked a button labeled EXIT GAMES on the games menu screen. Clicking the EXIT GAMES button cleared the games menu and returned the subject to the program of instruction at the point where he or she had left off. The component timer was not suspended while the subject played games, allowing the subject to "time-out" of a component and escape aversive response contingencies. The amount of time playing games during a component provided a precise measure of "off-task" behavior.

Performance summary. A summary screen (Figure 4) was displayed when the subject completed the set. The summary screen displayed the amount earned from the current set and the total amount earned from all sets. The current time was also displayed to facilitate the subject's judgment as to whether he or she had enough time to complete additional sets. Fiveminute breaks were also allowed between sets and this was indicated on the summary screen. $\underline{\text { Trial Block }}$

Prior to beginning the experiment, the potential subjects were told that they would first need to complete a trial session for which they would be paid $\$ 5.00$. Subjects were told that the trial session would help them to decide whether or not they felt that the task was something they would be willing to work on for an extended period of time. Prior to beginning the program the subject was asked to place any backpacks, purses, watches and cellular phones in a closet located outside of the experimental room. Subjects were then seated at the desk in front of the computer and given a "help file" (see Appendix C) for review. The experimenter remained with the subject while he or she read the "help file" to which they were referred if there were further 


\section{Earnings Summary:}

\section{CURRENT SESSION}

Earnings: $\$ 3.30$

Bonus: $\$ 0.15$

\section{TOTAL}

Earnings: $\$ 12.37$

Bonus: $\$ 2.10$

\section{The time is now 11:10 PM}

You may take a break now if needed (please no more than 5 minutes)

\section{EXIT SESSION}

Figure 4. Earnings summary presented to the subject at the completion of each set. Totals for the current set are displayed underneath the words "CURRENT SESSION" and the overall total is displayed under the words "TOTAL." 
questions about the task. Once the subject indicated that he or she was finished reading, a user name was provided that allowed the subject to log into the program. The volume of computer speakers was then increased to produce "white noise" that blocked out extraneous sounds that were occasionally present in adjacent rooms. The subject was then instructed to wear headphones that connected to the computer's audio system. The program was then started by the experimenter. A log-in screen appeared on the computer when the program was started that asked the subject to type his or her user name into a blank. The experimenter left the room when the subject had successfully logged into the program and the first set had started.

When the trial set was completed, the subject was seated outside of the experimental room while the experimenter examined the results. If subjects had obtained at least $\$ .30$ in bonus earnings and had completed the trial set with at least 60 percent of answers correct, subjects were then returned to the experimental room and asked if they would like to continue with the experiment. If they agreed, they were given an informed consent form (Appendix B) which they read and signed before continuing with the experiment. Four subjects agreed to continue with the experiment and two subjects decided against continuing after the first trial block. A third subject, described above, did not meet the minimum performance criteria for continuation.

\section{$\underline{\text { Daily Sessions }}$}

Subjects that remained in the experiment followed a similar routine to that of the trial session for the remaining sessions. Prior to beginning a session, the subjects placed watches, backpacks, and cellular phones in the closet. All experimental equipment was set up prior to the subject's arrival so that they only needed to put on the headphones and type in their user names to begin. Subjects completed from one to four sets per session. 


\section{Experimental Phases}

Baseline and experimental phases were designed to assess the effects of increasing magnitudes of response cost (punishment) for errors on the subject's answer accuracy, response topography, and duration of off-task behavior.

Baseline. A baseline of responding was first established for each subject against which the effects of punishment could be evaluated. Response contingencies were the same in all three components during the baseline phase. Every correct response added $\$ .07$ to the subject's total earnings, subjects were required to identify components, and there was no penalty for incorrect answers. These response contingencies were in effect during both the first cycle and review sequences of each set.

Experimental. After stable responding (described below) was achieved in the baseline phase, the experimental phase was begun. The value of correct answers was increased from $\$ .07$ to $\$ .09$ to offset losses from incorrect answers that would otherwise have significantly decreased the average earnings per session.

Response contingencies were unchanged for the baseline component while the contingencies were modified for the extinction and punishment components. During the extinction component (orange), money for correct answers could only be obtained during the first cycle while correct answers during review sequences did earn money. Only correct answers during the first cycle added money to the total during the punishment component (purple). In addition, a responsecost contingency was in effect for the punishment component where incorrect answers resulted in the loss of money (for an example, see Table 1). The amount of money subtracted from the total for each incorrect answer was initially $\$ .03$ for all subjects and remained at that value until responding was stable, and was then incremented to $\$ .09$ and finally to $\$ .15$. Losses were 
Table 1

Example Multiple-Schedule Contingencies and Correlated Stimuli During Experimental Phases

\begin{tabular}{lccccc}
\hline \multicolumn{2}{c}{ Component } & \multicolumn{2}{c}{ First Cycle } & & \multicolumn{2}{c}{ Review } \\
\cline { 5 - 6 } Color & Name & Correct & Incorrect & Correct & Incorrect \\
\hline Green & BL & $+\$ .09$ & -- & $+\$ .09$ & -- \\
Orange & EXT & $+\$ .09$ & -- & -- & -- \\
Purple & PUN & $+\$ .09$ & $-\$ .03$ & -- & $-\$ .03$ \\
\hline
\end{tabular}

Note. Money added to or subtracted from the subject's total earnings is indicated by a numerical total in cents (e.g., \$.09) preceded by a “+" or “-” respectively. Losses and gains were displayed next to each answer in the same format. The value subtracted for each incorrect response depended upon the experimental phase and was set at either $\$ .03, \$ .09$, or $\$ .15$. The value for correct responses was $\$ .07$ during the baseline phase and \$.09 during experimental phases. "--" indicate that there was no monetary consequence associated with an outcome. $\mathrm{BL}=$ baseline component; EXT = extinction component; PUN = punishment component. 
indicated by displaying the amount of money subtracted from the total next to the incorrect answer (.e.g., “-\$.03”).

\section{$\underline{\text { Stability Criteria }}$}

Each component of the experiment was carried out for a minimum of 10 sessions and until responding was judged to be stable based on the percent of correct answers per set and visual inspection. Calculations of the stability of accurate responding were based on the last six sessions of each phase, with the difference in the mean percent correct between the first 3 of the last 6 sessions and the last 3 of the last 6 sessions being no more than 0.10 . Visual inspection of stability was based on the presence or absence of upward or downward trend (i.e., increasing or decreasing answer accuracies) across consecutive sets.

\section{$\underline{\text { Summary }}$}

The general strategy of the present experiment was to establish a stable baseline of responding in all components against which the effects of extinction and punishment for incorrect answers could be examined. Efforts were taken to isolate the effects of response contingencies to the components in which those contingencies were in effect. For example, punishment for incorrect answers during component 3 was expected to have specific effects that could be identified during the punishment component, while the effects of extinction would be apparent during the extinction component. Of primary interest were changes in answer accuracy, due to the problems noted with the occurrence of errors during learning. Additional changes in the subject's behavior that may correlate with answer accuracy, including changes in the rate at which the subject progresses through the program, were also of interest and examined. 


\section{Chapter 4}

\section{Results}

Descriptive and quantitative analyses of each subject's responding during the program of instruction were conducted to determine if extinction and punishment following incorrect responses reliably increased answer accuracy and decreased the overall response rate. Multiple response measures provided the means for evaluating changes in responding that contributed to the overall experimental effects.

\section{$\underline{\text { Response Measures }}$}

Of primary interest for analysis were changes in the overall accuracy of answers during the program of instruction as a function of the presence or absence of reinforcement and punishment contingencies. The accuracy of each subject's answers was therefore assessed throughout the experiment. Other measures contributed to the analysis of answer accuracy, including subjects' use of edit keys (BACKSPACE and DELETE) when composing answers. This measure was examined to identify changes in the effort that subjects applied toward constructing a correct response as a function of the contingencies for doing so. Changes in the rate at which the subject progressed through the program were also evaluated by examining the amount of time that subjects spent engaging in the tasks associated with completing individual frames. These tasks involved reading text and typing answers to a frame, examining the status of answers submitted for evaluation, and examining correct answers when incorrect answers had been provided. Response measures associated with individual answers were also evaluated, including the average time between each keystroke used to construct an answer. It was anticipated that through examination of these response measures a comprehensive picture of the effects of extinction and punishment during learning could be obtained. 


\section{$\underline{\text { Naming Conventions }}$}

\section{$\underline{\text { Components }}$}

At least two different components of the MULT schedule were in effect for every session for every subject. Each of the components and their corresponding response contingencies were treated as isolated entities in the present analysis. During the first phase of the experiment (the baseline phase; see additional description below) the response contingencies within each component were the same: all correct answers resulted in money, and subjects were given as many opportunities as needed to provide a correct answer. The names given to the components, however, preempted the changes in response contingencies that were instated during later experimental phases. For example, "punishment component" was used to describe the component in which punishment in the form of response-cost for incorrect answers was implemented and correct answers earned money only on the first attempt. This descriptor was used for this component for all phases of the experiment, even for the baseline phase of the experiment when the punishment contingencies were not in effect. Similarly, the component during which only correct answers provided on the first attempt earned money was described as the "extinction component" prior to the implementation of those contingencies (i.e., during the baseline phase). "Baseline component" was used to describe the component during which response contingencies remained the same throughout all phases of the experiment: during the baseline component all correct answers resulted in money being added to the subject's total, irrespective of the number of attempts required.

\section{$\underline{\text { Baseline and Experimental Phases }}$}

The term "baseline component" is used solely in the context of component descriptions and analyses, and is not synonymous with the term "baseline phase." The baseline phase was the 
first phase of the experiment, and consisted of a minimum of ten consecutive sessions for each subject. During the baseline phase, an attempt was made to establish stable responding during each component of the MULT schedule: baseline, extinction, and punishment. Following the baseline phase, the response contingencies in the extinction and punishment components were altered, as described above, while the response contingencies in the baseline component remained unchanged. The phases of the experiment that followed the baseline phase are referred to as "experimental phases," as it was during these phases that the experimental manipulation of response contingencies within the extinction and punishment components were instated.

\section{$\underline{\text { Data Analysis and Presentation }}$}

\section{$\underline{\text { Visual Inspection }}$}

Data from the present study are presented in graphical format and tabular format when appropriate. Graphs and tables provide visual summaries of the data that allow for the immediate interpretation of the effects of experimental manipulations. Each of the data sets that are presented, however, contain large numbers of observations that justify a statistical analysis to corroborate visual inspection of results. An emphasis was placed on obtaining effects detectable through visual rather than statistical procedures, however. This emphasis is due to the fact that results that are detectable by visual inspection are likely to suggest methods and procedures relevant to taking practical action with respect to the subject matter (Sidman, 1960).

\section{$\underline{\text { Statistical Analyses }}$}

Each subject in the present experiment was continuously responding as the treatment variables (extinction and punishment) were repeatedly withdrawal and then applied. This type of experimental design is amenable to nonparametric statistical analyses, especially those outlined by Siegel (1956). In the present study, differences in responding between components were 
assessed using the nonparametric Wilcoxon matched-pairs signed-ranks test, a variation of the sign-test. This test was chosen based on the justification provided by Edginton (1982) and Munson and Crosbie (1998) regarding differences in the sensitivity of the sign-test and the Wilcoxon matched-pairs signed-ranks test.

The sign-test allows for the determination of an experimental effect when the procedure involves repeated measures and the treatment variable (e.g., punishment) continuously alternates with a phase or phases where the treatment variable is absent. To conduct an analysis with the sign- test, repeated observations obtained with the treatment variable in effect are paired with observations obtained when the treatment variable was absent. Of interest is the presence of a difference (positive or negative) between these observations over repeated measurements. If the cumulative differences among the pairs of observations (e.g., more negative differences than positive differences) is larger than would be expected by chance alone, the treatment variable can be assumed to have had an effect. While the sign-test can be used to determine the direction of difference between repeated observations (e.g., more positive differences were obtained than negative differences), the Wilcoxon matched-pairs signed-ranks test can be used to determine the relative magnitude of those differences as well. This is accomplished by weighting larger differences between pairs of observations more heavily than smaller differences. As the size of the differences could be determined for all measures obtained in the present study, the Wilcoxon matched-pairs signed-ranks test was used to determine the presence of differences among matched pairs of observations and the relative magnitude of those differences within an experimental phase.

$\underline{\text { Units of Analysis }}$

An experimental session was defined as the completion of a set of frames from the program 
of instruction. Each set varied in the number of frames and therefore so did the number of responses (from 28 to 108 per set). The length of a session varied accordingly, depending on the number of frames. Component times within each set did not vary, lasting six minutes each. Due to the variability of the length of a session and the invariability of the length of a component, there were sessions where few responses, or no responses, occurred during one of the components. For example, set 34 consisted of 25 frames and 28 responses. A subject could complete this session in less than or slightly more than 12 minutes, thus allowing time only for two of the three components to be in effect for that session. Statistical comparisons of responding during each of the three components would not be possible, as the Wilcoxon matched-pairs signed-ranks test requires that there be a comparable value (i.e., matched-pair) in each component for analysis. To resolve this issue, responses during each component within each phase were grouped together in a continuous chain, from the first measurement in the phase to the last measurement, in the order of occurrence. The resulting chain was then divided into bins of a fixed size (e.g., ten answers). Each of the bins was then collapsed to a single data point (i.e., observation). This data point served as one half of a matched pair; the other half of the matched pair was a bin of data from a different component. For example, S02 (subject S02) provided a total of 674 answers during the first phase of the experiment (the baseline phase). Of those answers, 221 were made during the baseline component, 209 during the extinction component, and 244 during the punishment component. Assuming a bin size of ten answers, the number of possible bins for the baseline component was 22 (221 / 10), with 20 (209 / 10) bins available for the extinction component, and 24 (244 / 10) bins available for the punishment component. The extinction and punishment components were components in which there was an experimental manipulation of response consequences, while in the baseline component response 
consequences were never changed. Performance during the extinction and punishment components was therefore separately compared to the performance during the baseline component. The number of actual bins that could be compared (i.e., matched pairs) was determined by the smaller number of bins for the two components under consideration. From the above example, there were a total of 20 bins for comparison as matched pairs for the baseline and extinction components, and a total of 22 bins for comparison as matched pairs for the baseline and punishment components. Throughout the present analysis the number of observations within each bin varied for different response measures, but the procedure for constructing bins was always the same.

\section{$\underline{\text { Frame Cycles }}$}

Each session was completed only when correct answers had been provided for every answer to every frame. Ensuring that every answer to every frame was correct required the subject to provide answers to some frames more than once. The procedure by which subjects were given multiple opportunities to provide correct answers to a frame involved continuously cycling through a set of frames until all answers were correct. On each successive cycle, those frames where only correct answers had been provided were removed until none were left and the set was completed. However, only the performance data generated during the first cycle through a set of frames was included in the present analysis.

\section{Procedural Change}

Subjects were earning on average less than $\$ 5.00$ per hour during the baseline phase of the experiment and therefore less than had been advertised during recruitment. As a result, interest in continuing with the project was noticeably affected (e.g., subjects began skipping sessions). Interest in the project was expected to further decline when the amount of money earned was 
decreased through the extinction and punishment contingencies that subtracted from each subject's average session earnings. The value of each correct answer was therefore increased from $\$ .07$ to $\$ .09$ following transition from the baseline to the experimental phases. This procedural change introduced an experimental confound: differences in performance between the baseline and experimental phases could be attributed to an increase in the value of each correct response rather than to the introduction of the extinction and punishment contingencies per se. An attempt was made to correct for this problem by 1) exposing each subject to at least two values of the punishment contingency, and 2) significantly increasing the amount of money lost when the subject transitioned to an experimental phase that required a higher punishment value. Thus, rather than increase the punishment value by one cent after achieving stability in an experimental phase, the punishment value was increased by six cents (e.g., from $-\$ .03$ to $-\$ .09$ ). By greatly increasing the punishment value between experimental phases, changes in responding between experimental phases and within components could reliably be attributed to the greater magnitude of the punishment contingency rather than its presence or absence alone. This procedure increased the importance of comparisons of responding during punishment components as the value of the punishment contingency increased between experimental phases. Comparisons of responding when extinction and punishment contingencies were absent (during the initial baseline phase) versus present (later experimental phases) are likewise deemphasized.

A visual summary of each subject's earnings throughout the experiment is presented in Table 2. The structure of this table will be described in detail because a similar format will be used for presenting other tabular data. The first column on the left contains each subject's identification symbol that was assigned when he or she began the experiment. Experimental phases are listed in the second column. Phases are listed in the order in which they were implemented, from top 
Table 2

Average Earnings for All Phases

Average Session Earnings

\begin{tabular}{ccccccc}
\cline { 3 - 5 } Subject & & & & & & \\
& & & & & & \\
Phase & Sessions & Set & Answer & Bonus & Session \\
\hline \multirow{3}{*}{ S02 } & & & & & & \\
& BL & 9 & 3.16 & .070 & .66 & 3.82 \\
& P.03 & 11 & 4.14 & .068 & .95 & 5.09 \\
S04 & P.09 & 13 & 3.33 & .062 & .66 & 3.99 \\
& & & & & & \\
& BL & 9 & 3.28 & .070 & .54 & 3.82 \\
S05 & P.03 & 12 & 3.46 & .059 & .69 & 4.15 \\
& P.09 & 14 & 2.58 & .056 & .51 & 3.09 \\
& BL & 10 & 3.29 & .070 & .72 & 4.01 \\
& P.03 & 14 & 3.65 & .062 & .92 & 4.57 \\
& P.09 & 10 & 2.79 & .066 & .59 & 3.38 \\
& P.15 & 11 & 2.40 & .056 & .48 & 2.88 \\
& BL & 12 & 3.40 & .070 & .67 & 4.07 \\
& P.03 & 10 & 3.95 & .067 & .86 & 4.81 \\
& P.09 & 14 & 2.58 & .057 & .63 & 3.21 \\
\hline
\end{tabular}

Note. All values displayed for average session earnings are in dollar amounts. Average earnings are those obtained for every set of the program, every answer within a set, total bonus per set, and the average per set in addition to the average bonus per set (i.e., session total). BL = baseline; P $=$ punishment, with suffixed values indicating the amount of money subtracted for incorrect responses in the experimental phase (e.g., -\$.03). 
to bottom. Each subject was first exposed to the baseline phase, labeled "BL," prior to advancing to the experimental phases, labeled with a "P" and followed by a decimal equivalent of the amount of money lost for incorrect responses. For example, "P.03" indicates that the corresponding row values were obtained during an experimental phase where the extinction and punishment contingencies were in effect, and the value of the punisher was set at $-\$ .03$. Other punishment values were $-\$ .09$ and $-\$ .15$ (subject $\mathrm{S} 05$ only). The third column of Table 2 lists the number of sessions (equivalent to the number of sets) completed and analyzed within an experimental phase. For example, data for nine baseline sessions were available for S02, followed by 11 sessions within an experimental phase where the punishment value was set at $\$ .03$. A total of 13 sessions were completed by S02 when the punishment value was increased to -\$.09. Data in the remaining columns of Table 2 are specific to the present analysis and provide the average amount earned for each session, for each answer within a session, and for the bonus contingency (+\$.15 for selecting the colored square corresponding to the current component). The final column lists the overall average earnings for each session. Of particular interest within Table 2 is the average amount of money earned for each answer following the increase in value of a correct response from $\$ .07$ to $\$ .09$ (when the subject transitioned from the "BL" to "P.03"). Even with the increase of $\$ .02$ for each correct answer, the overall average earnings per answer decreased when the extinction and punishment contingencies were in effect. The overall earnings per session increased during the first experimental phase ("P.03") for all subjects relative to the baseline phase, however, and this was due to the fact that the sets of the instructional program contained many more frames during this phase than previous sets during the baseline phase. While the average earnings per answer decreased during the experimental phases, the average session earnings initially increased due to the larger number of frames in a 
session.

$\underline{\text { Baseline Data }}$

Each phase of the experiment was anticipated to have at least ten sessions for analysis.

However, the computer used for the present experiment could not simultaneously run all of the programs necessary for the program of instruction, data analysis, and storage. The result was that some data were lost. This problem was detected early and then corrected, but data loss occurred for S04, for whom the data for sessions two and three were not stored. This subject completed a total of 12 baseline sessions, leaving a total of nine sessions for analysis (the first session was conducted only for trial purposes and wasn't included in the present analysis for any subject).

Due to an experimental error, S02 was transitioned from the baseline phase after completing a total of nine sessions rather than ten, so that both S02 and S04 have a total of nine baseline sessions rather than the minimally anticipated 10 sessions for analysis. As the following overall analysis will demonstrate, it is unlikely that the absence of these data altered the overall conclusions of the experiment.

\section{Experimental Analyses}

$\underline{\text { Stability }}$

Responding during each phase of the experiment was required to meet criterion measures of stability before the subject was transitioned to another phase. Stability was defined as a difference of no greater than 10 percent between the average number of correct answers for the last 3 and first 3 of the last 6 sessions of a phase within the experiment. Additionally, visual inspection of the data had to reveal an absence of trend (a tendency towards increasingly accurate or inaccurate responding) across the final six sessions (see Table 2). Stability values 
obtained throughout the experiment are displayed in Table 3. Values ranged from .01 to .10, with an average stability value of .04 , indicating that for all subjects the transitions between the experimental phases were conducted only after responding met the pre-determined stability criteria.

\section{Component identification}

Subjects were given the chance to earn a bonus of $\$ .15$ by correctly selecting the color associated with the component currently in effect. At randomly chosen times during a session a screen appeared containing three colored squares and a directive to select the current "component color" with the mouse. The accuracy with which subjects selected the correct color is reported in Table 4. Reported in the table are the average number of attempts per session, the average number of correct identifications, and the average percent correct identifications. Three of the four subjects obtained averages above 90 percent accuracy, and S04 obtained an average 74 percent accuracy. The overall average for correct identifications for all subjects across all phases of the experiment was .93. These data indicate that subjects reliably attended to the component-correlated stimuli throughout all phases of the experiment, but obtained values that were considerably lower than those obtained by previous researchers (e.g., Madden \& Perone, 1999).

\section{Correct Answers}

The accuracy of each answer that subjects provided was evaluated by the program and immediate feedback was given to the subject on the status of each answer. A summary of each subject's accuracy at providing correct answers is displayed graphically in Figure 5. The number of correct answers in each component is displayed. Each data point indicates the number of correct answers within a bin of ten consecutive answers in each component. Of interest was the 
Table 3

Measures of Stability For All Phases

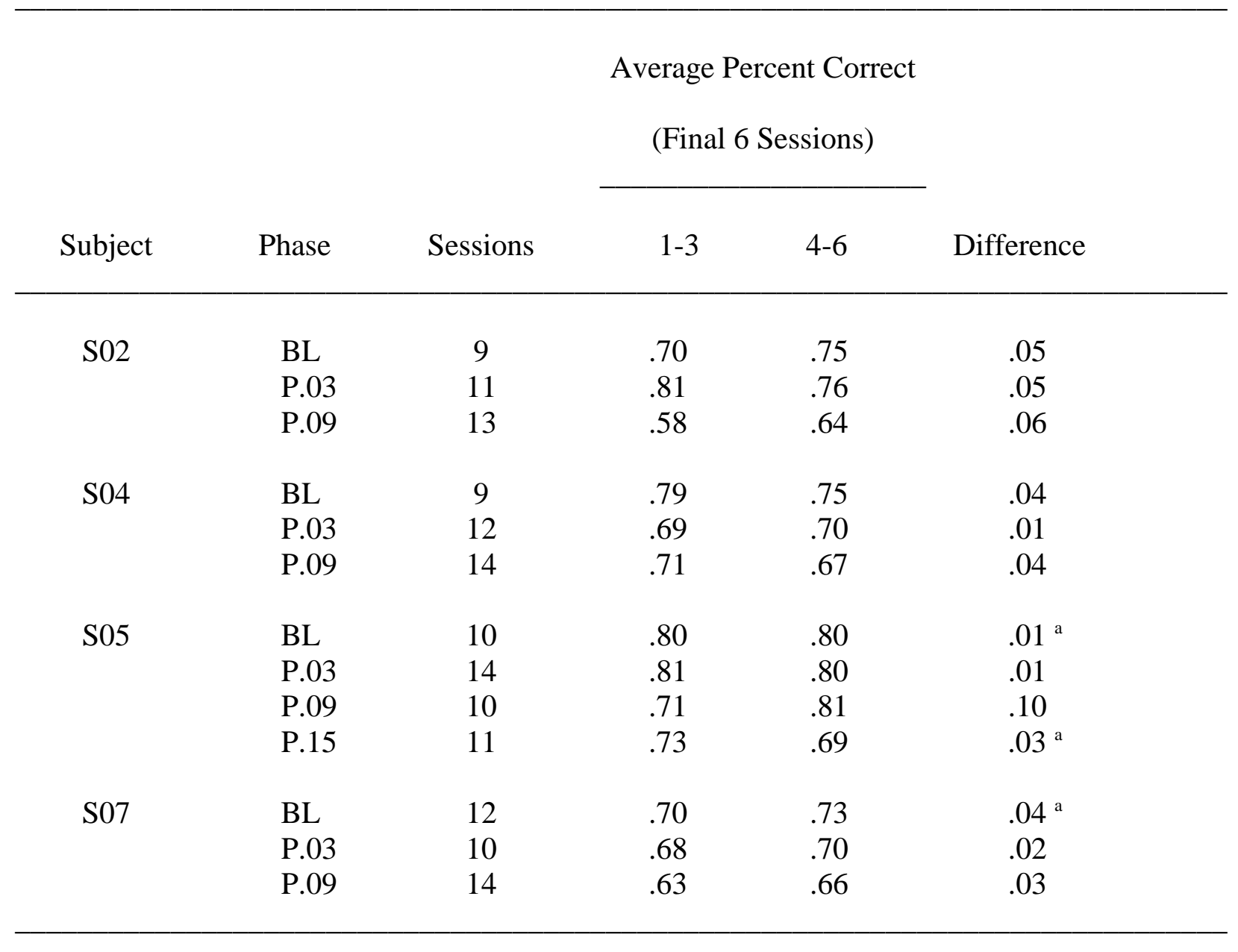

Note. Stability is based on the average percent correct of the last six sessions for each phase. Stability values (DIFFERENCE) indicate the absolute value of the difference between the mean of the first three sessions and the mean of the last three sessions from the final six sessions in a phase. $\mathrm{BL}=$ baseline; $\mathrm{P}=$ punishment, with suffixed values indicating the amount of money subtracted for incorrect responses in the experimental phase (e.g., $-\$ .03$ ).

${ }^{a}$ Rounding affects the observed agreement between stability measures and differences between the averages shown in the table. 
Table 4

Accuracy at Selecting the Stimulus Correlated with the Component in Effect

\begin{tabular}{|c|c|c|c|c|c|}
\hline \multirow[b]{2}{*}{ Subject } & \multirow[b]{2}{*}{ Phase } & \multirow[b]{2}{*}{ Sessions } & \multicolumn{3}{|c|}{ Session Averages } \\
\hline & & & Opportunities & Correct & Percent \\
\hline \multirow[t]{3}{*}{ S02 } & BL & 9 & 4.70 & 4.40 & .94 \\
\hline & P.03 & 11 & 6.55 & 6.36 & .97 \\
\hline & P.09 & 13 & 4.69 & 4.38 & .93 \\
\hline \multirow[t]{3}{*}{ S04 } & BL & 9 & 4.92 & 3.58 & .73 \\
\hline & P.03 & 12 & 6.33 & 4.58 & .72 \\
\hline & P.09 & 14 & 4.43 & 3.43 & .77 \\
\hline \multirow[t]{4}{*}{ S05 } & BL & 10 & 5.00 & 4.82 & .96 \\
\hline & P.03 & 14 & 6.36 & 6.14 & .97 \\
\hline & P.09 & 10 & 4.00 & 3.90 & .98 \\
\hline & P.15 & 11 & 4.09 & 3.18 & .78 \\
\hline \multirow[t]{3}{*}{ S07 } & BL & 12 & 5.15 & 4.46 & .87 \\
\hline & P.03 & 10 & 6.30 & 5.70 & .90 \\
\hline & P.09 & 14 & 4.43 & 4.21 & .95 \\
\hline
\end{tabular}

Note. The average number of attempts per session is reported as well as the average number of correct selections and the overall percent. All percent values are significantly above chance. BL = baseline $; \mathrm{P}=$ punishment, with subscript values indicating the amount of money subtracted for incorrect responses in the experimental phase (e.g., $-\$ .03$ ). 

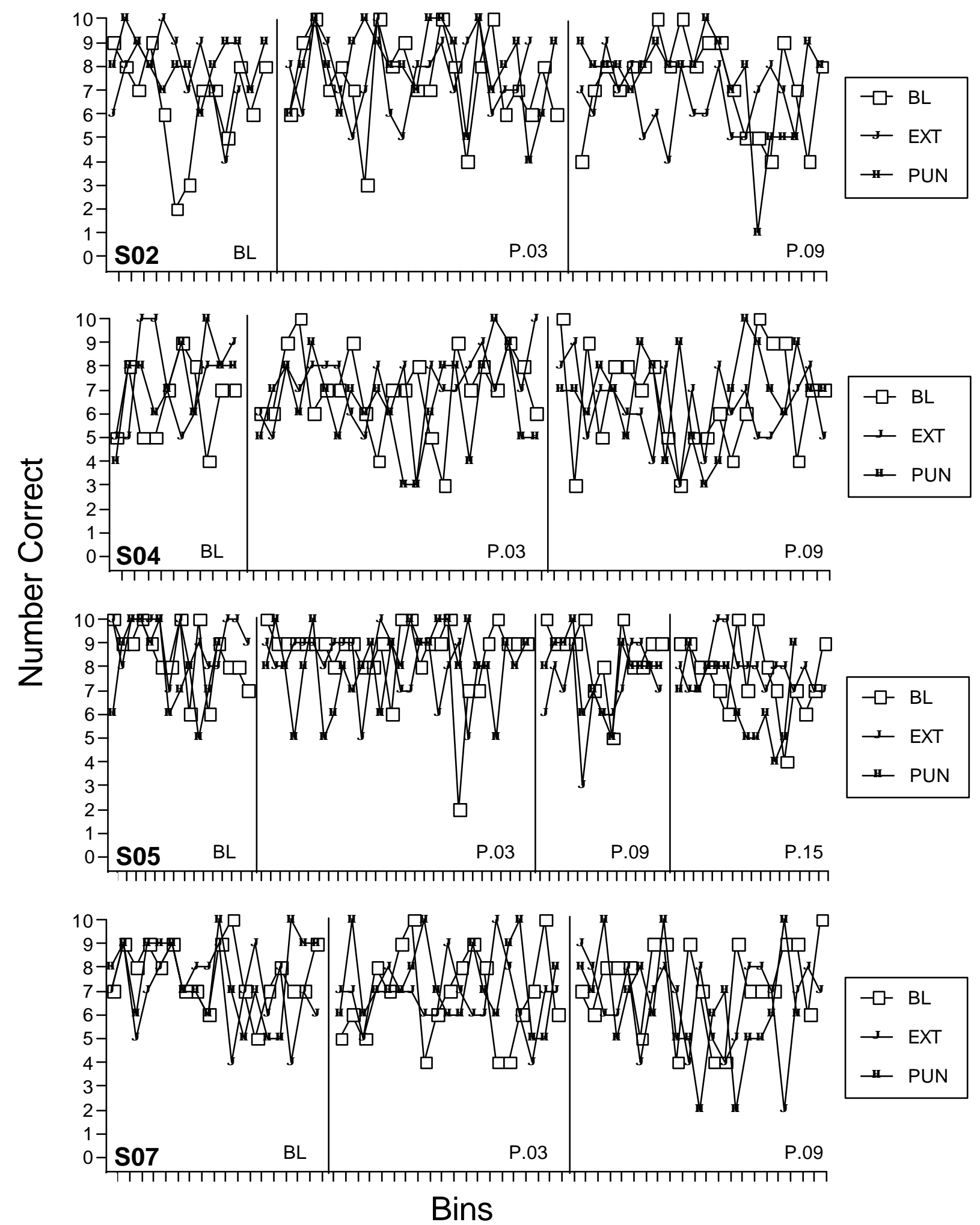

Figure 5. Number of correct answers from bins of ten consecutive answers in each component. P.03/ P.09 / P.15 = experimental phases; $\mathrm{BL}=$ baseline component; $\mathrm{EXT}=$ extinction component; $\mathrm{PUN}=$ punishment component. 
change in the number of correct answers per bin within each component. During the baseline phase, all correct answers earned money, and there was no limit to the number of attempts that were possible. During the experimental phases (e.g., "P.03"), an unlimited number of opportunities to earn money for correct answers remained in effect only during the baseline component; during the extinction component money could only be earned during the first cycle; otherwise there was no monetary consequence for a correct response. During the punishment component, money could only be earned during the first cycle and all incorrect answers resulted in the loss of money (e.g. -\$.03). Visual inspection of Figure 5 reveals that extinction and punishment response contingencies present during experimental phases did not produce reliable differences in answer accuracy between components for any subject. For S02 during phase P.09, the number of accurate responses obtained during the extinction component for bins 6 through 13 is consistently lower than the number of accurate responses obtained during the baseline and punishment components, but this difference disappears by bin 14. Differential effects of extinction and punishment contingencies on answer accuracy between components may have been present but undetectable for S05 due to a ceiling effect: this subject's accuracy was very high, and improvements in accuracy during any component could only be small and difficult to detect.

Across subjects and phases, a decrease in answer accuracy is evident. This observation is confirmed by examining the values of the average percent of correct answers during each component of each phase, as listed in Table 5. Inspection of this table reveals that accuracy of responding in each component in each phase decreases for all subjects across experimental phases. A single exception is noted for S02 during the baseline phase relative to the later experimental phases which in turn show a decline. The range of differences in answer accuracy 
Table 5

$\underline{\text { Average Percent Correct Across Experimental Phases }}$

Component Percent Correct

\begin{tabular}{llllll} 
Subject & Phase & BL & EXT & PUN & Average \\
\hline S02 & & & & & \\
& BL & .68 & .78 & .82 & .76 \\
& P.03 & .74 & .77 & .80 & .77 \\
S04 & P.09 & .73 & .64 & .74 & .70 \\
& & & & & \\
& BL & .70 & .76 & .73 & .73 \\
S05 & P.03 & .69 & .72 & .64 & .68 \\
& P.09 & .66 & .62 & .68 & .65 \\
& & & & & .84 \\
& BL & .84 & .88 & .80 & .83 \\
S07 & P.03 & .86 & .81 & .81 & .78 \\
& P.15 & .84 & .72 & .77 & .74 \\
& BL & .76 & .78 & .68 & .75 \\
& P.03 & .77 & .71 & .76 & .70 \\
& P.09 & .68 & .68 & .74 & .66 \\
\hline
\end{tabular}

Note. P.03 / P.09 / P.15 = experimental phase; EXT = extinction component; PUN = punishment component; $\mathrm{BL}=$ baseline component. 
between the baseline phase and the final experimental phase completed for each subject is -.06 percent to -.10 percent, with an average decrease of -.08 percent.

A summary of the statistical comparisons of answer accuracy for all subjects is presented in Table 6. The data presented in Table 6 are based on the Wilcoxon matched-pairs signed-ranks test, with values located in the column labeled " $\underline{N}$ " indicating the number of matched-pairs used for each comparison and the values in the column labeled " $T$ " indicating the obtained value of the comparison. Identical analyses were conducted for each response measure obtained throughout the experiment. Of interest in the present analysis was the effect of the response contingencies present during the extinction and punishment components relative to the baseline component where response contingencies had not been altered. The effects of the experimental manipulations were therefore assessed by examining each subject's performance during the baseline component relative to the subject's performance in the extinction component and punishment component. For S05, the number of correct answers obtained during the extinction component was significantly lower than the number of correct answers obtained during baseline component during phase P.09, $T(\underline{\mathrm{N}}=11)=9.0, \mathrm{p}<.05$; this difference is not consistent between phases, however.

A cumulative record of correct answers is displayed in Figure 6. Data points indicate the cumulative sum of correct answers. These sums were obtained by adding successive bin values from each component within each experimental phase. Slopes of lines indicate how quickly correct answers were added to the cumulative number of correct answers. Differences in the slopes of the lines within each phase indicate differential control of accurate responding by extinction and punishment contingencies. Slope differences are detectable for S02 during the baseline phase, where correct answers were added to the cumulative total more rapidly during 
Table 6

$\underline{\text { Statistical Comparison of Answer Accuracy Across Experimental Phases }}$

\begin{tabular}{|c|c|c|c|c|c|c|c|}
\hline \multirow[b]{3}{*}{ Subject } & \multirow[b]{3}{*}{ Component } & \multicolumn{6}{|c|}{ Experimental Phase } \\
\hline & & \multicolumn{2}{|c|}{ P.03 } & \multicolumn{2}{|c|}{ P.09 } & \multicolumn{2}{|c|}{ P.15 } \\
\hline & & $\underline{N}$ & $T$ & $\underline{N}$ & $T$ & $\underline{N}$ & $T$ \\
\hline \multirow[t]{2}{*}{ S02 } & EXT & 17 & 71.0 & 15 & 33.5 & & \\
\hline & PUN & 17 & 55.5 & 13 & 43.0 & & \\
\hline \multirow[t]{2}{*}{ S04 } & $\mathrm{EXT}$ & 19 & 86.0 & 20 & 90.0 & & \\
\hline & PUN & 19 & 66.5 & 17 & 64.5 & & \\
\hline \multirow[t]{2}{*}{ S05 } & EXT & 17 & 51.0 & 11 & $9.0^{*}$ & 13 & 41.0 \\
\hline & PUN & 22 & 92.0 & 7 & 2.5 & 11 & 16.0 \\
\hline \multirow[t]{2}{*}{ S07 } & EXT & 17 & 64.0 & 18 & 58.5 & & \\
\hline & PUN & 17 & 64.0 & 19 & 67.0 & & \\
\hline
\end{tabular}

Note. Comparison is of the number of correct answers from bins of ten consecutive answers in each component. Each bin comprised a single observation. Responding during the extinction and punishment components were compared with responding during the baseline component. P.03 / P.09 / P.15 = experimental phase; $\underline{N}=$ number of matched pairs of observations; $T=$ value obtained from the Wilcoxon matched-pairs signed-ranks test; EXT = extinction component; PUN = punishment component.

$* \underline{p}<.05$. 

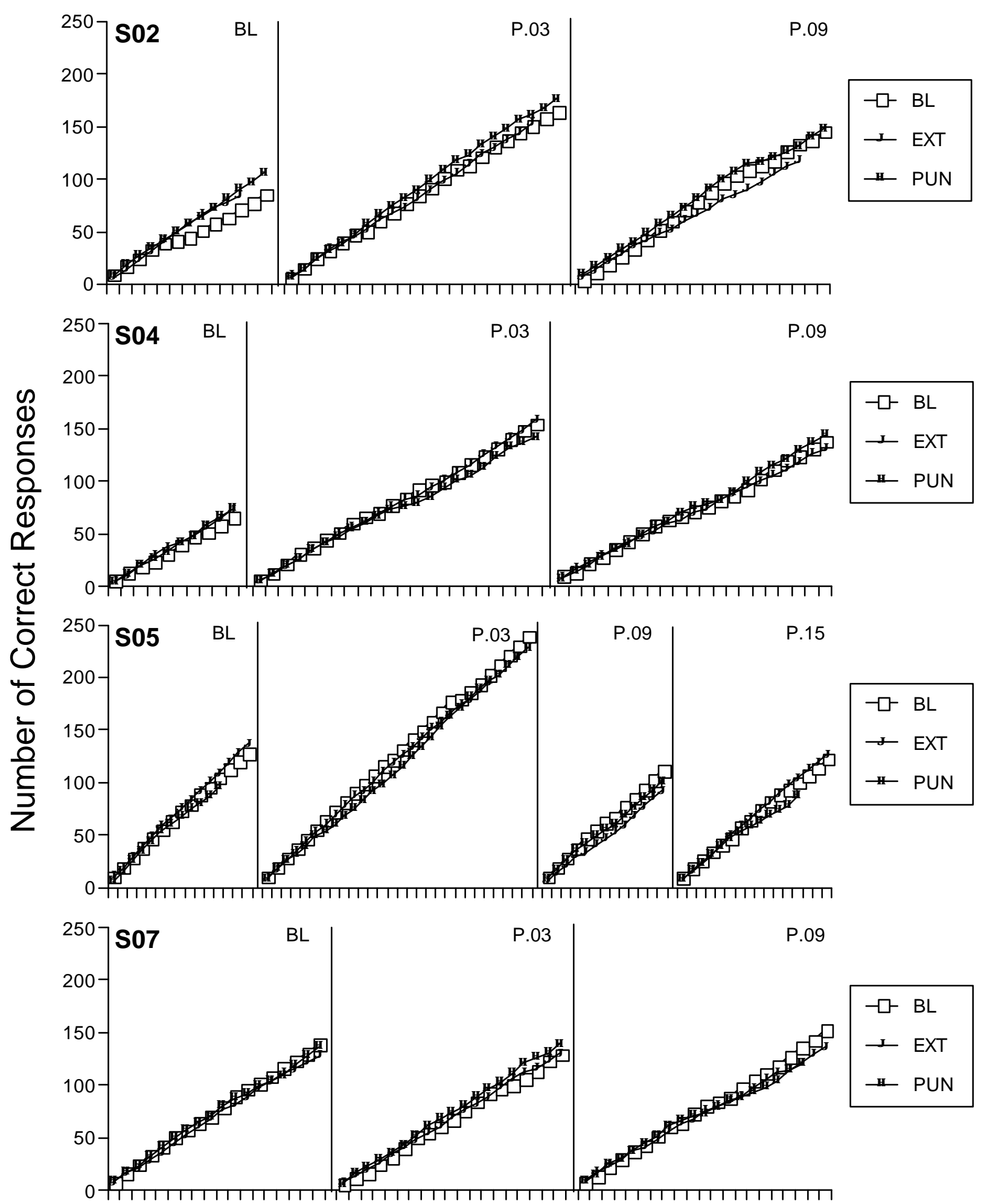

Bins

Figure 6. Cumulative number of correct answers from bins of ten consecutive answers in each component. P.03 / P.09 / P.15 = experimental phases; $\mathrm{BL}=$ baseline component; EXT = extinction component; PUN $=$ punishment component. 
the extinction component and punishment component compared to the baseline component, although the reason for this is unclear as there were no differences in response contingencies during the baseline phase. Slope differences are also detectable for S05, where the cumulative total is greater during the baseline component than for either the extinction or punishment components during phases P.03 and P.09. During phase P.15 the number of correct answers for S05 appears to decrease during the punishment component relative to other components. As previously noted, only the difference the baseline and extinction components during phase P.09 were statistically significant for this subject (see Table 6).

Figure 7 displays the average number of correct answers for every bin of ten answers obtained in each component in each experimental phase. This visual display corroborates the data presented in Table 5 and provides accuracy information relevant to each component. A trend towards decreasing answer accuracy is noticeable for all subjects. Consistent differences in accuracy across components are visible only for S05, where the average number of correct responses during the punishment component is lower than those during the baseline component across all experimental phases.

\section{$\underline{\text { Edit Keys }}$}

Subjects used the edit keys on their keyboard (BACKSPACE and DELETE) to modify their answers prior to submitting them for evaluation. The cumulative numbers of edit keys used for ten consecutive answers in each component were summed into discrete bins. Figure 8 displays the number of edit keys within each of these bins. An increase in the number of edit keys used across experimental phases is visible for S02, S05, and S07. This increase does not appear to be systematically related to the component in effect for S05, but there is evidence of a relation between the use of edit keys and component contingencies for S02 and S07. For S02, the 


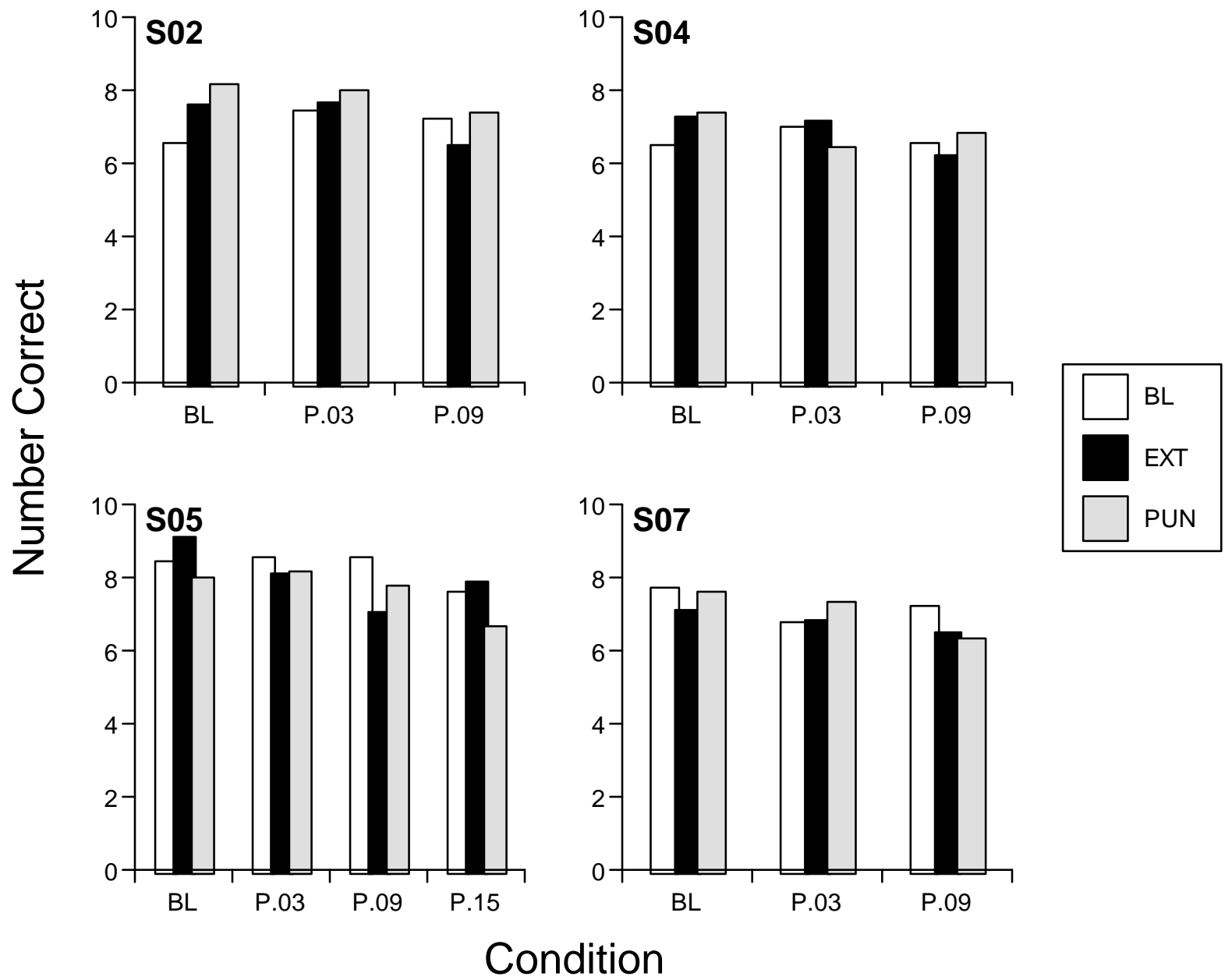

Figure 7. Average number of correct answers for bins of ten consecutive answers in each component. P.03 / P.09 / P.15 = experimental phases; $\mathrm{BL}=$ baseline component; EXT = extinction component; $\mathrm{PUN}=$ punishment component. 

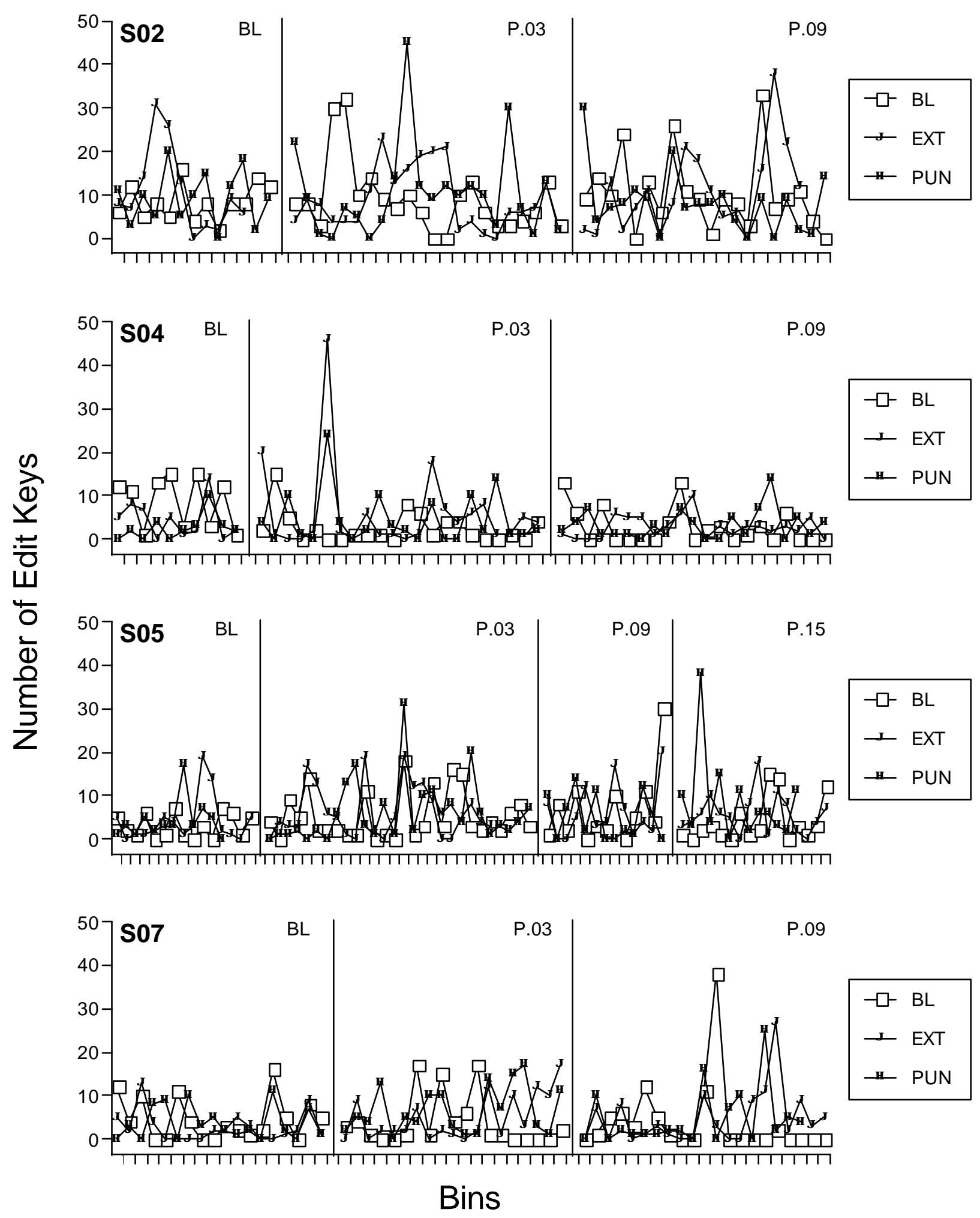

Figure 8. The number of edit keys (BACKSPACE and DELETE) used in each component when composing answers. Each bin contains the sum of the number of edit keys for ten cosecutive answers in each component. P.03 / P.09 / P.15 = experimental phases; BL = baseline component; EXT = extinction component; $\mathrm{PUN}=$ punishment component. 
number of edit keys used during phase P.03 is higher during the extinction component than the baseline component for bins 8 through 13, but this difference is not maintained. For bins 9 through 13 of the same phase, the number of edit keys used during the punishment component is also higher than the number obtained during the baseline component. This difference disappears following bin 13, however. For S07, the number of edit keys used during the extinction component of phase P.03 is higher than the number used during the baseline component for bin 13 and bins 15 through 19. A similar increase in the number of edit keys used during the punishment component relative to the baseline component of phase P.03 is found for bins 13 through 17, and for bin 19. These differences for S07 disappear following transition to phase P.09. The number of edit keys used by S07 during phase P.09 increases during the punishment component relative to the baseline component for bins 13,14 , and 16 through 19 . At the same time, the number of edit keys used during the extinction component is larger than the number used during the baseline component for bins 15 through 21 . The use of a greater number of edit keys during the extinction and punishment components by S07 is suggestive but is not reliable across phases. As indicated in Table 7, the results of statistical comparisons among these components are not significant.

Examination of the average number of edit keys used by each subject during all experimental phases (see Figure 9) indicates that a small but visible increase in the number of edit keys used across experimental phases for S02, S05, and S07, but not S04, for whom the average number of edit keys used decreased across phases. An average increase in the use of edit keys is especially notable for S05, for whom there is an increase across phases. Only for S07 does the average increase in the use of edit keys occur within the extinction and punishment components relative to the baseline component. 
Table 7

$\underline{\text { Statistical Comparison of the use Edit Keys Across Experimental Phases }}$

\begin{tabular}{|c|c|c|c|c|c|c|c|}
\hline \multirow[b]{2}{*}{ Subject } & \multirow[b]{2}{*}{ Component } & \multicolumn{2}{|c|}{ P.03 } & \multicolumn{2}{|c|}{ P.09 } & \multicolumn{2}{|c|}{ P.15 } \\
\hline & & $\underline{N}$ & $T$ & $\underline{N}$ & $T$ & $\underline{N}$ & $T$ \\
\hline \multirow[t]{2}{*}{ S02 } & EXT & 20 & 102.5 & 18 & 78.5 & & \\
\hline & PUN & 19 & 80.5 & 19 & 62.0 & & \\
\hline \multirow[t]{2}{*}{ S04 } & EXT & 16 & 42.5 & 17 & 74.0 & & \\
\hline & PUN & 22 & 94.0 & 19 & 86.5 & & \\
\hline \multirow[t]{2}{*}{ S05 } & EXT & 23 & 107.0 & 12 & 36.5 & 16 & 44.0 \\
\hline & PUN & 27 & 178.5 & 13 & 45.0 & 12 & 18.5 \\
\hline \multirow[t]{2}{*}{ S07 } & EXT & 18 & 73.5 & 15 & 44.5 & & \\
\hline & PUN & 18 & 53.0 & 15 & 48.5 & & \\
\hline
\end{tabular}

Note. Comparison is of the number of edit keys (BACKSPACE and DELETE) used to modify a typed answer. The number of edit keys used for ten consecutive answers were summed into a bin to create a single observation in each component. Responding during the extinction and punishment components were compared with responding during the baseline component. P.03 / P.09 / P.15 = experimental phase; $\underline{\mathrm{N}}=$ number of matched pairs of observations; $T=$ value obtained from the Wilcoxon matched-pairs signed-ranks test; EXT = extinction component; PUN $=$ punishment component. None of the differences between the components were significantly different. 


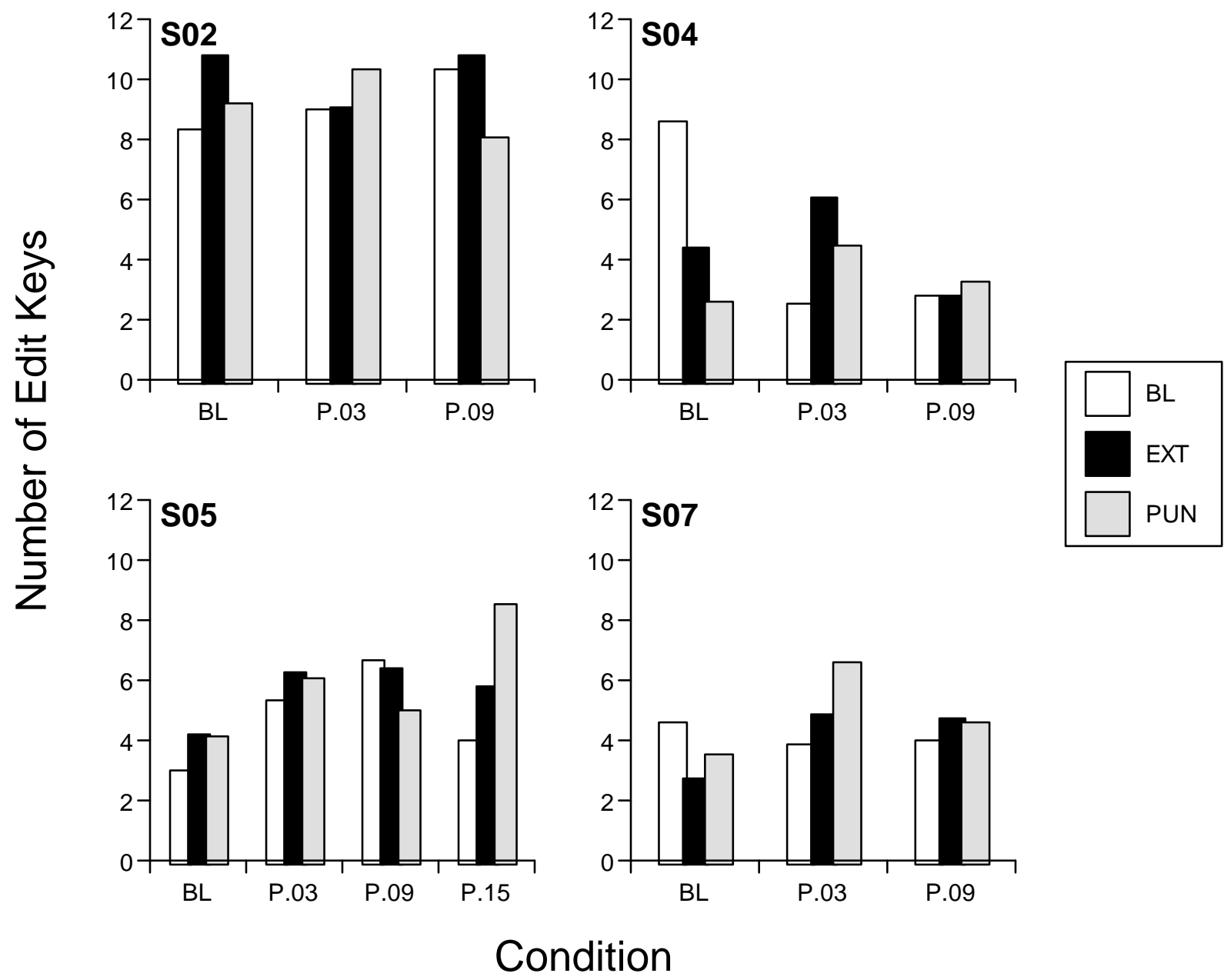

Figure 9. The average number of edit keys used for every ten consecutive answers in each component. P.03 / P.09 / P.15 = experimental phases; $\mathrm{BL}=$ baseline condition; $\mathrm{EXT}=$ extinction condition; $\mathrm{PUN}=$ punishment condition. 


\section{$\underline{\text { Keystroke Latency }}$}

The average amount of time in seconds between the keystrokes used to compose an answer was used as a measure of response latency. The sum of latency averages for ten answers comprised a single bin for analysis. Higher cumulative latencies indicated slower typing (i.e., a decrease in rate). Variability for this measure was low and differences in the latencies between components were not readily detectable through visual inspection as indicated by examination of Figure 10. The latencies between keystrokes appears to increase during extinction and punishment components relative to the baseline component for S07 during phase P.03, and a reversal of this finding appears during phase P.09.

The average cumulative keystroke latencies across bins of 10 answers are presented in Figure 11. For S05, the keystroke latency generally decreases across phases and the latency of keystrokes during the extinction component is consistently lower than the baseline component. For two of the three experimental phases the latency of keystrokes during the punishment component is lower than the latency during the baseline component. For S07 the latencies of keystrokes during both the extinction and punishment components is greater than for the baseline component. As indicated in Table 8, the difference in keystroke latencies between the baseline and punishment components for S05 during phase P.09 are significant, as are the differences in keystroke latencies between the baseline and extinction components for S07 during phase P.03. In summary, none of the obtained differences were reliable.

\section{$\underline{\text { Viewing Correct Answers }}$}

If the answer a subject provided was judged to be incorrect, the correct answer could be viewed by pressing a number key on the keyboard that corresponded to the number of the answer. The total amount of time in seconds that each subject spent viewing correct answers 

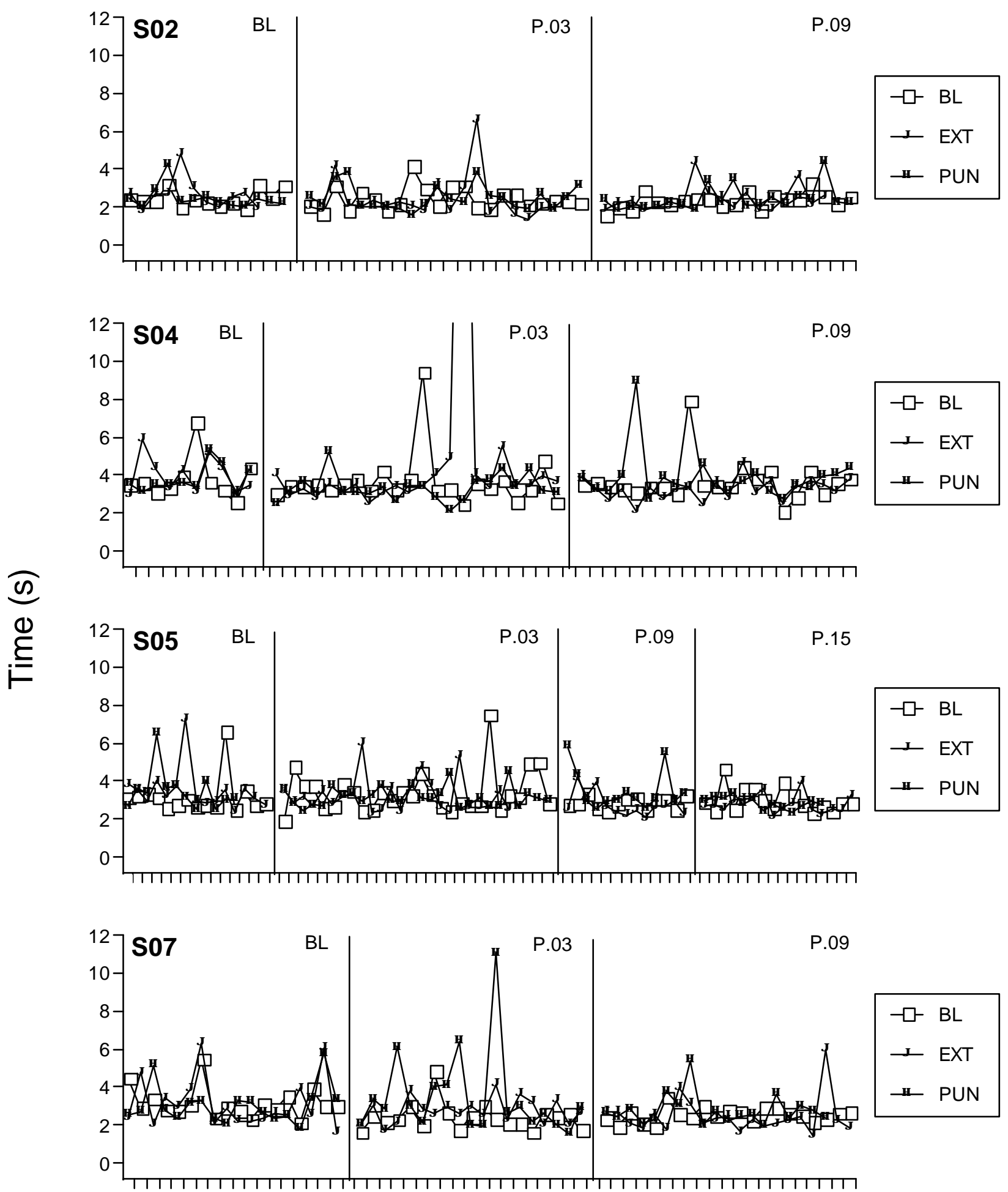

\section{Bins}

Figure 10. The sum of the average latencies between keystrokes used to compose individual answers. The average latencies for ten answers were summed into bins for each component. P.03 / P.09 / P.15 = experimental phases; $\mathrm{BL}=$ baseline component; EXT = extinction component; $\mathrm{PUN}=$ punishment component. 


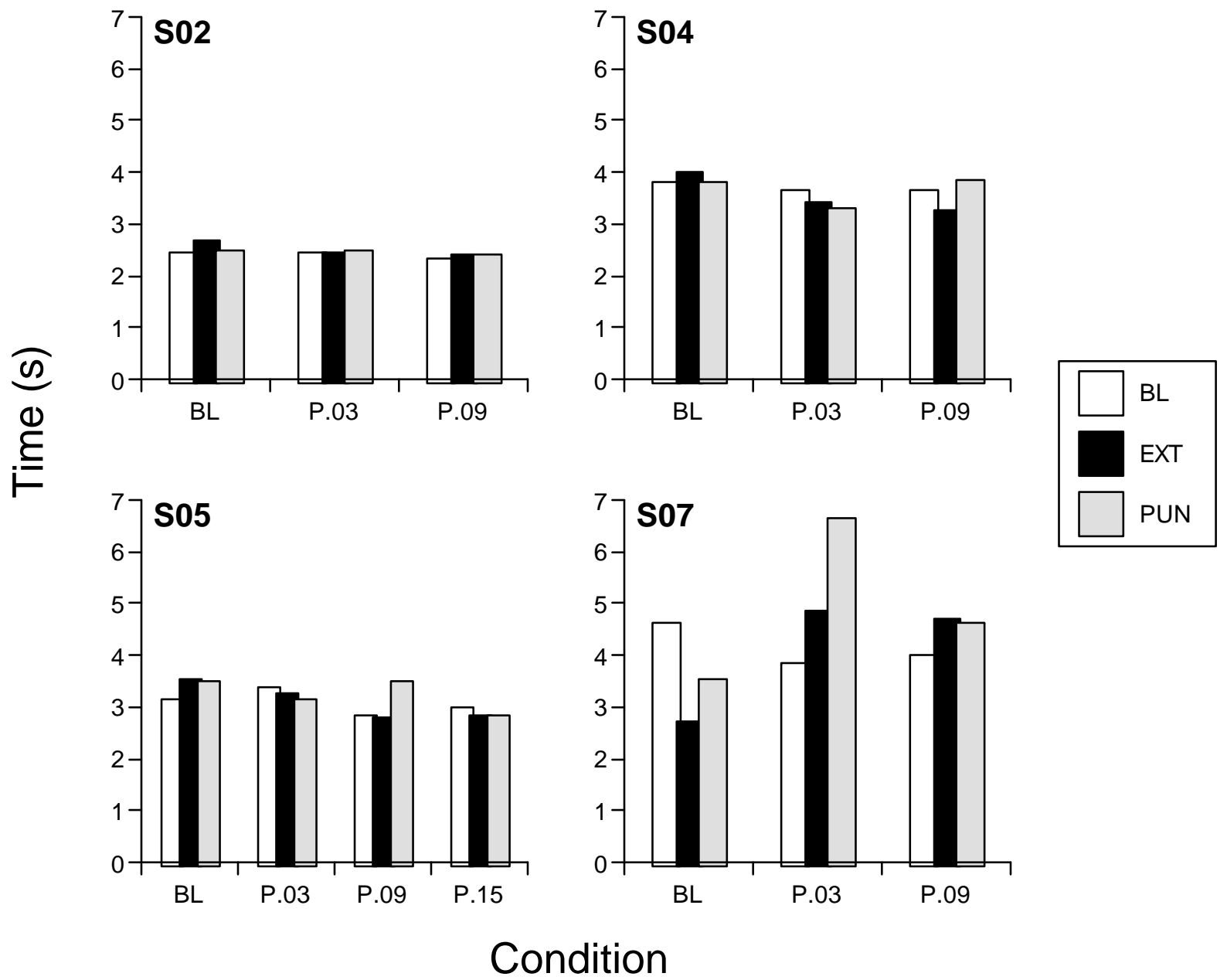

Figure 11. The average sum of the average latencies between keystrokes used to compose individual answers. Bins indicate the average latencies for ten consecutive answers in each component. Bars indicate the average obtained bin size. P.03 / P.09 / P.15 = experimental phases; $\mathrm{BL}=$ baseline component; $\mathrm{EXT}$ = extinction component; $\mathrm{PUN}=$ punishment component. 
Table 8

$\underline{\text { Statistical Comparison of Latencies Between Key Strokes }}$

\begin{tabular}{|c|c|c|c|c|c|c|c|}
\hline \multirow[b]{3}{*}{ Subject } & \multirow[b]{3}{*}{ Component } & \multicolumn{6}{|c|}{ Experimental Phase } \\
\hline & & \multicolumn{2}{|c|}{ P.03 } & \multicolumn{2}{|c|}{ P.09 } & \multicolumn{2}{|c|}{ P.15 } \\
\hline & & $\underline{N}$ & $T$ & $\underline{N}$ & $T$ & $\underline{\mathrm{N}}$ & $T$ \\
\hline \multirow[t]{2}{*}{ S02 } & EXT & 19 & 75.5 & 16 & 58.0 & & \\
\hline & PUN & 22 & 117.5 & 20 & 98.0 & & \\
\hline \multirow[t]{2}{*}{ S04 } & EXT & 22 & 101.0 & 20 & 62.5 & & \\
\hline & PUN & 23 & 108.0 & 20 & 79.5 & & \\
\hline \multirow[t]{2}{*}{ S05 } & EXT & 23 & 136.5 & 13 & 36.0 & 16 & 55.0 \\
\hline & PUN & 27 & 169.0 & 12 & $9.0 * *$ & 12 & 28.0 \\
\hline \multirow[t]{2}{*}{ S07 } & EXT & 19 & $45.5^{*}$ & 20 & 77.0 & & \\
\hline & PUN & 17 & 36.5 & 18 & 55.0 & & \\
\hline
\end{tabular}

Note. Comparison is of the average latency in seconds between each keystroke used when typing an answer. The average latency for ten answers was summed to obtain a single observation. Responding during the extinction and punishment components were compared with responding during the baseline component. P.03 / P.09 / P.15 = experimental phase; $\underline{N}=$ number of matched pairs of observations; $T=$ value obtained from the Wilcoxon matched-pairs signedranks test; $\mathrm{EXT}=$ extinction component; $\mathrm{PUN}=$ punishment component.

$* \underline{p}<.05 . * * \underline{p}<.02$. 
when answers were incorrect was summed into bins for analysis. Each bin consisted of the total amount of time spent viewing a correct answer across three consecutive incorrect answers within each component. Each of these bins is presented graphically as a data point in Figure 12. The data presented in Figure 12 are highly variable across subjects, as indicated by the different ordinate axes scales for each subject. Visual inspection of the data indicate that for all subjects there is a trend towards spending less time reviewing correct answers with corresponding decreases in variability. This trend is particularly evident for S05 and S07, but is also detectable for S02 and S04. Between-component differences in the time spent reviewing correct answers are visible for S04 and S07. During phase P.09, S04 spent more time viewing the correct response during the punishment component than the baseline component for bins 7 through 13, after which this difference becomes unreliable. During phase P.03, the amount of time that S07 spent reviewing correct answers during the extinction component was less than the amount of time spent during the baseline component for bins 15 through 20. A similar pattern is detectable during phase P.09 for the same subject, where the extinction component time remains below the baseline component time for bins 4 through 8 . This difference becomes unreliable after bin 8 in phase P.09, however.

Table 9 presents a summary of the statistical analyses used to evaluate these data. Significant results were obtained for S04 who spent more time viewing correct answers during the punishment component than the baseline component during phase P.09, corroborating differences detectable through a visual inspection of these data. During phase P15, S05 spent significantly less time viewing the correct response during the extinction component than the baseline component and significantly more time viewing the correct response during the punishment component than the baseline component. 

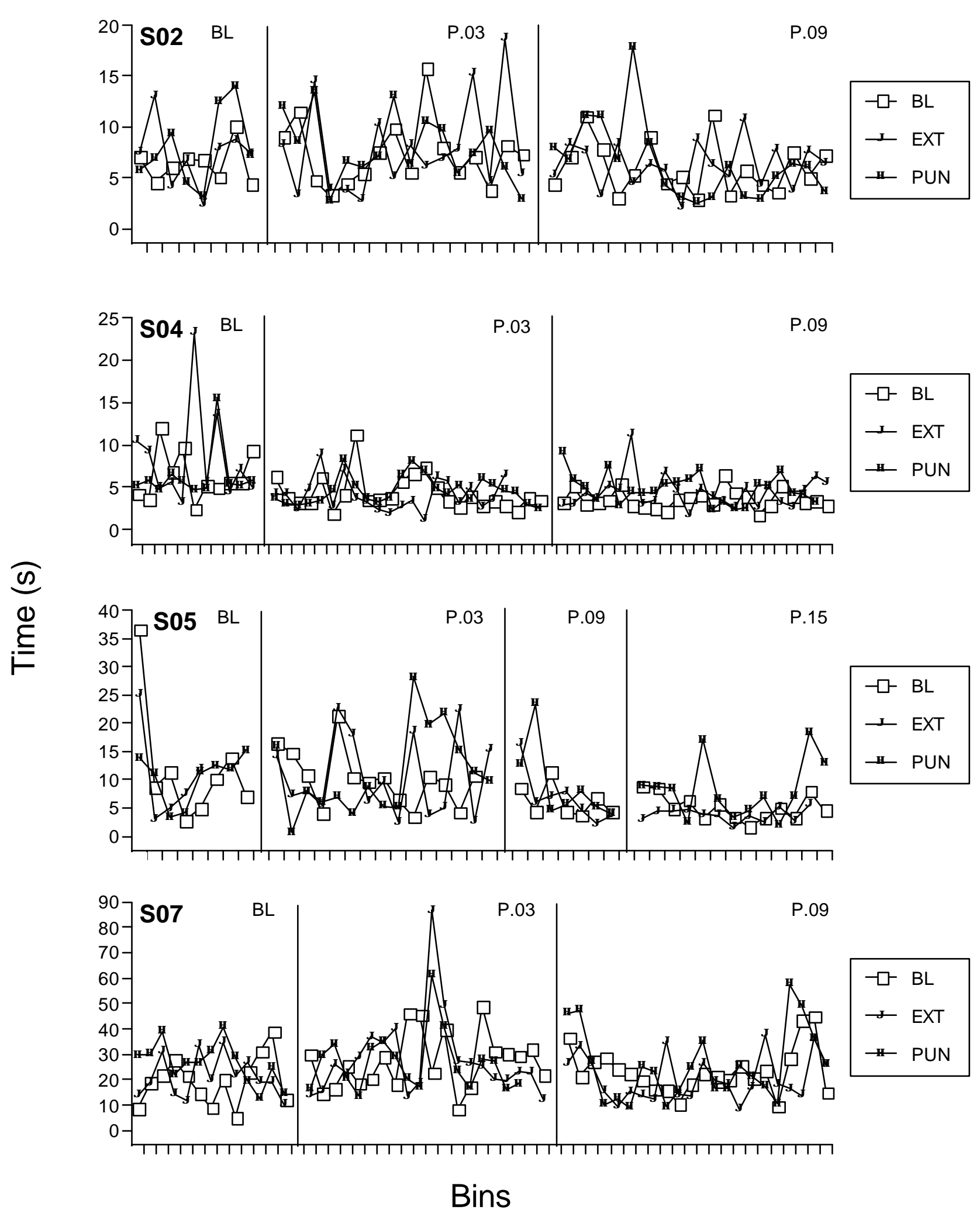

Figure 12. The sum of the time in seconds that subjects spent reviewing the correct answer when an answer was incorrect. The times for three consecutive reviewed answers were summed into bins for each component. P.03 / P.09 / P.15 = experimental phases; $\mathrm{BL}=$ baseline component; EXT = extinction component; $\mathrm{PUN}=$ punishment component. Note differences in the scales of ordinate axes. 
Table 9

$\underline{\text { Statistical Comparison of Time Spent Reviewing Correct Answers }}$

\begin{tabular}{|c|c|c|c|c|c|c|c|}
\hline \multirow[b]{3}{*}{ Subject } & \multirow[b]{3}{*}{ Component } & \multicolumn{6}{|c|}{ Experimental Phase } \\
\hline & & \multicolumn{2}{|c|}{ P.03 } & \multicolumn{2}{|c|}{ P.09 } & \multicolumn{2}{|c|}{ P.15 } \\
\hline & & $\underline{N}$ & $T$ & $\underline{N}$ & $T$ & $\underline{\mathrm{N}}$ & $T$ \\
\hline \multirow[t]{2}{*}{ S02 } & EXT & 16 & 61.0 & 17 & 67.0 & & \\
\hline & PUN & 16 & 54.0 & 18 & 79.0 & & \\
\hline \multirow[t]{2}{*}{ S04 } & EXT & 21 & 113.5 & 24 & 104.0 & & \\
\hline & PUN & 22 & 101.0 & 23 & $61.0 * *$ & & \\
\hline \multirow[t]{2}{*}{ S05 } & EXT & 14 & 43.0 & 7 & 12.0 & 12 & $13.5^{*}$ \\
\hline & PUN & 14 & 50.5 & 7 & 10.0 & 13 & $17.0^{*}$ \\
\hline \multirow[t]{2}{*}{ S07 } & EXT & 20 & 102.5 & 22 & 93.0 & & \\
\hline & PUN & 18 & 81.0 & 22 & 102.0 & & \\
\hline
\end{tabular}

Note. Comparison is of the cumulative amount of time in seconds spent viewing a correct answer when an answer had been evaluated as incorrect. The total time for three answers was summed to obtain a single observation. Responding during the extinction and punishment components were compared with responding during the baseline component. P.03 / P.09 / P.15= experimental phase; $\underline{\mathrm{N}}=$ number of matched pairs of observations; $T=$ value obtained from the Wilcoxon matched-pairs signed-ranks test; EXT = extinction component; PUN = punishment component.

$* \underline{p}<.05 . * * \underline{p}<.02$. 
Figure 13 presents the average value of each bin within each component of each experimental phase. Examination of Figure 13 suggests a trend of decreasing amounts of time spent viewing correct answers across phases of the experiment for S02, S05, and S07. For S02 and S07, an initial increase in the average amount of time spent reviewing correct answers appears during phase P.03, and that increase is followed by a decrease during phase P.09. An additional observation is that for S02, S04, and S05, the average amount of time spent viewing correct answers is greater during the punishment component than either the extinction component or the baseline component. The exception to this finding is provided by S07, whose viewing time is less during the punishment component than the baseline component during phase P.03.

\section{Composition Time}

The composition time for a frame was defined as the amount of time that elapsed between the appearance of a new frame on the computer screen and the subject's submission of answers for evaluation by pressing the RETURN key on the keyboard. The composition times for ten consecutive frames within each component were summed into individual bins for analysis. Each bin value is presented as an individual data point in Figure 14, the examination of which reveals data of a highly variable nature with no obvious patterns of distribution. A possible exception is a slight increase in composition time for S04, S05, and S07 during phase P.03 relative to the baseline phase. The lack of experimental effects detectable through visual inspection with this data set are confirmed through statistical analyses (presented in Table 10) that reveal no significant differences between comparisons.

The average length of time for each bin of composition times is presented in Figure 15. Differences in composition times between components are present but small during phase P.03 

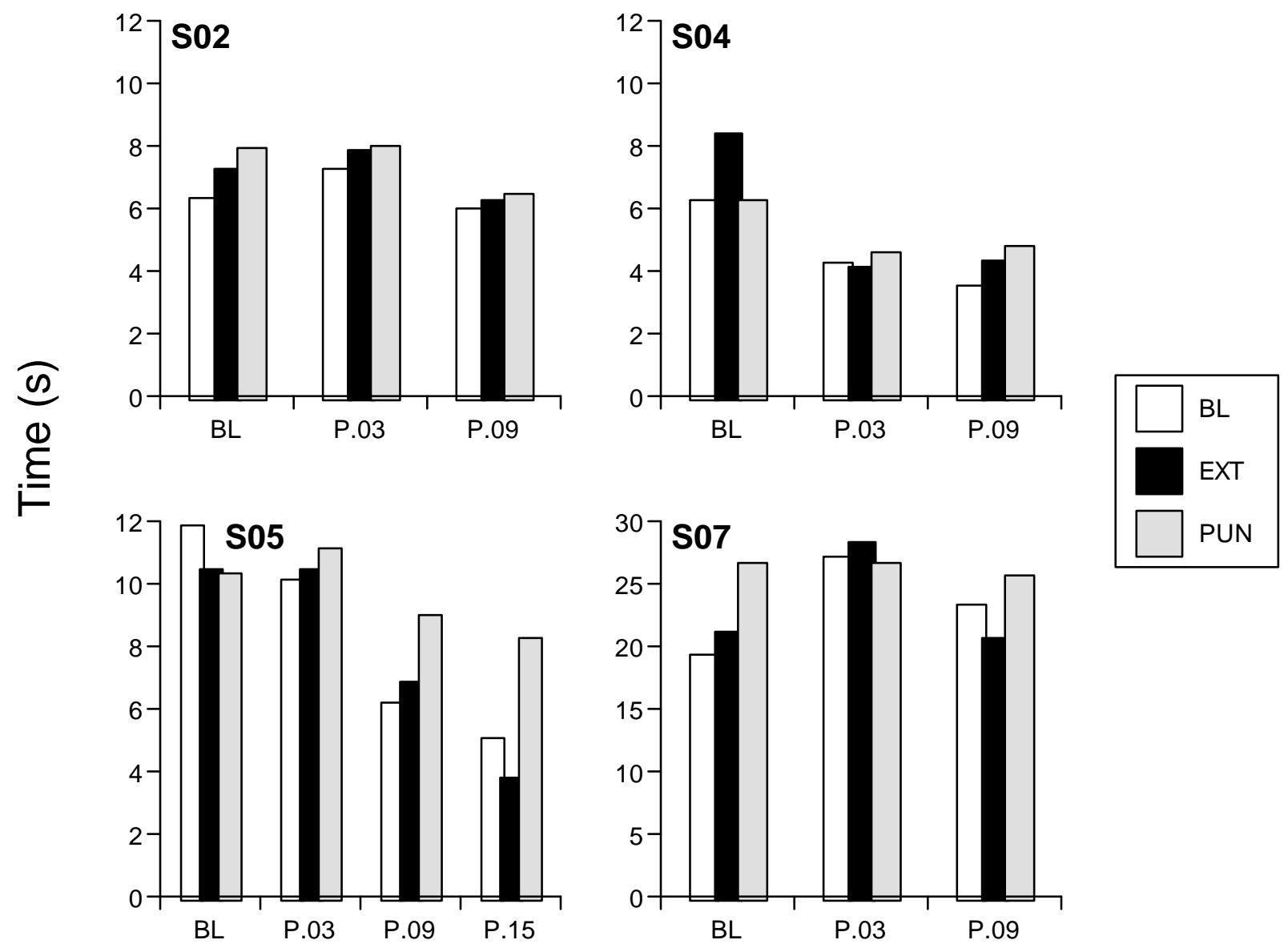

\section{Condition}

Figure 13. The average sum of the time in seconds that subjects spent reviewing the correct answer when an answer was incorrect. The times for three consecutive reviewed answers were summed into bins for each component. Bars indicate the averages of those sums. P.03 / P.09 / P.15 = experimental phases; $\mathrm{BL}=$ baseline component; $\mathrm{EXT}=$ extinction component; $\mathrm{PUN}=$ punishment component. Note differences in the scales of ordinate axes. 


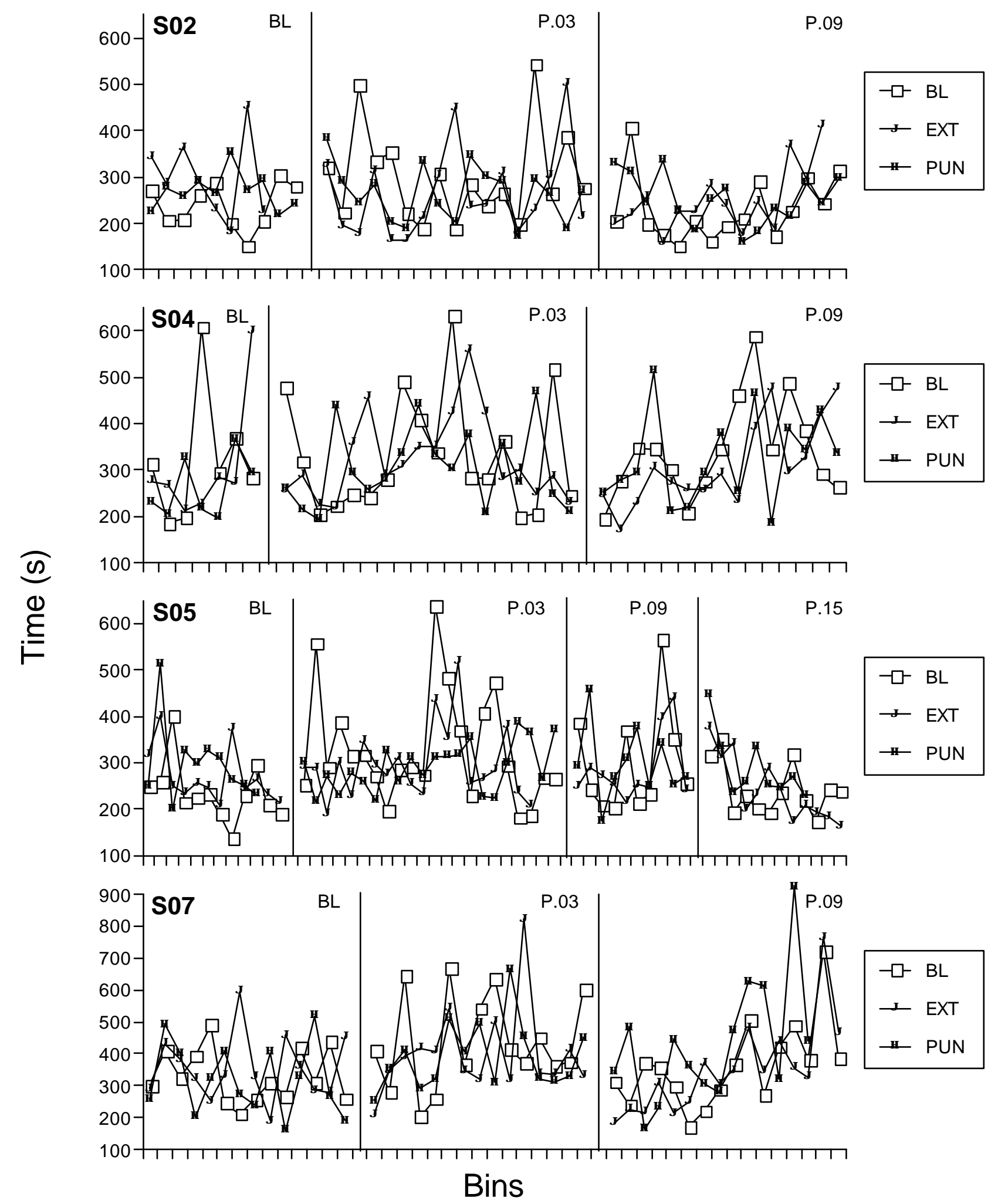

Figure 14. The sum of the time in seconds that subjects spent reading text and composing answers.

The times for ten consecutive frames were summed into bins for each component. P.03 / P.09 / P.15 = experimental phases; $\mathrm{BL}=$ baseline component EXT = extinction component; $\mathrm{PUN}=$ punishment component. Note differences in the scales of ordinate axes. 
Table 10

$\underline{\text { Statistical Comparison of Time Spent Composing Answers }}$

\begin{tabular}{|c|c|c|c|c|c|c|c|}
\hline \multirow[b]{3}{*}{ Subject } & \multirow[b]{3}{*}{ Component } & \multicolumn{6}{|c|}{ Experimental Phase } \\
\hline & & \multicolumn{2}{|c|}{ P.03 } & \multicolumn{2}{|c|}{ P.09 } & \multicolumn{2}{|c|}{ P.15 } \\
\hline & & $\underline{N}$ & $T$ & $\underline{N}$ & $T$ & $\underline{N}$ & $T$ \\
\hline \multirow[t]{2}{*}{ S02 } & EXT & 17 & 56.0 & 14 & 33.0 & & \\
\hline & PUN & 17 & 58.0 & 15 & 46.0 & & \\
\hline \multirow[t]{2}{*}{ S04 } & EXT & 18 & 83.0 & 15 & 45.0 & & \\
\hline & PUN & 19 & 83.0 & 14 & 43.0 & & \\
\hline \multirow[t]{2}{*}{ S05 } & EXT & 20 & 72.0 & 10 & 27.0 & 12 & 39.0 \\
\hline & PUN & 22 & 91.0 & 10 & 25.0 & 9 & 9.0 \\
\hline \multirow[t]{2}{*}{ S07 } & EXT & 15 & 42.0 & 16 & 58.0 & & \\
\hline & PUN & 15 & 41.0 & 14 & 25.0 & & \\
\hline
\end{tabular}

Note. Comparison is of the cumulative amount of time in seconds spent reading frame material and composing answers in each component prior to submitting answers for evaluation. The total time for ten frames was summed to obtain a single observation. Responding during the extinction and punishment components were compared with responding during the baseline component. P.03 / P.09 / P.15 = experimental phase; $\underline{\mathrm{N}}=$ number of matched pairs of observations; $T=$ value obtained from the Wilcoxon matched-pairs signed-ranks test; EXT = extinction component; PUN = punishment component. None of the differences between the components were significantly different. 

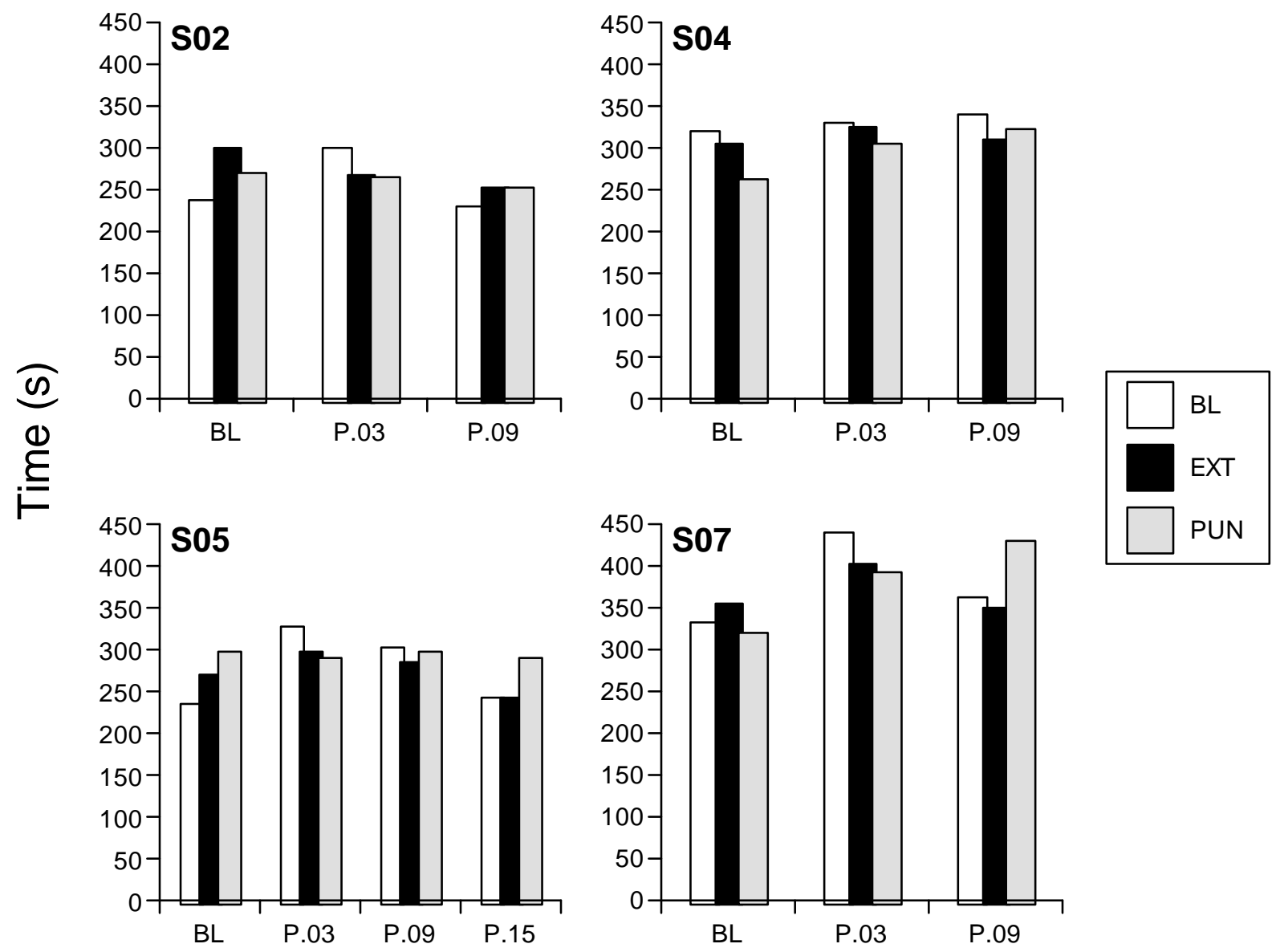

\section{Condition}

Figure 15. The average sums of the time in seconds that subjects spent reading text and composing answers. The times for ten consecutive frames were summed into bins for each component. Bars indicate the average of those sums. P.03 / P.09 / P.15 = experimental phases; $\mathrm{BL}=$ baseline component; $\mathrm{EXT}$ = extinction component; $\mathrm{PUN}=$ punishment component. 
for all subjects, where the average composition time is longer during baseline components than extinction components. During phase P.03 the average composition time during extinction components is longer than the average composition times during punishment components for all subjects. Overall decreases in average composition times were also evident across experimental phases (but not baseline phases) for S02, S05, and S07.

\section{$\underline{\text { Review Time }}$}

Feedback was provided as to the accuracy of each answer immediately after subjects submitted their answers for evaluation. The period of time that elapsed between the appearance of feedback on the screen and the subject's press of the SPACEBAR on the keyboard to advance to the next frame was defined as the review time. The review times for ten consecutive frames within each component were summed into individual bins for analysis. Each bin is presented as an individual data point in Figure 16. Visual inspection of these data suggest that for some subjects there were reliable differences in review times between components. For example, review times for S02 during phase P.09 were higher during the extinction component than the baseline component for bins 5 through 12. During all experimental phases for S05 (i.e., P.03, P.09, and P.15) review times during the punishment component "spike" above or nearly above the highest surrounding review time values obtained during either the extinction component or the punishment component. As indicated in Table 11, the review time lengths obtained during the punishment component were significantly higher than the review time lengths obtained during the baseline component only during phase P.09 for S05. No other comparisons were statistically significant.

The average amount of time per bin of answer review times is displayed in Figure 17. Consistent differences between subjects are not evident from visual inspection. For S05, the 

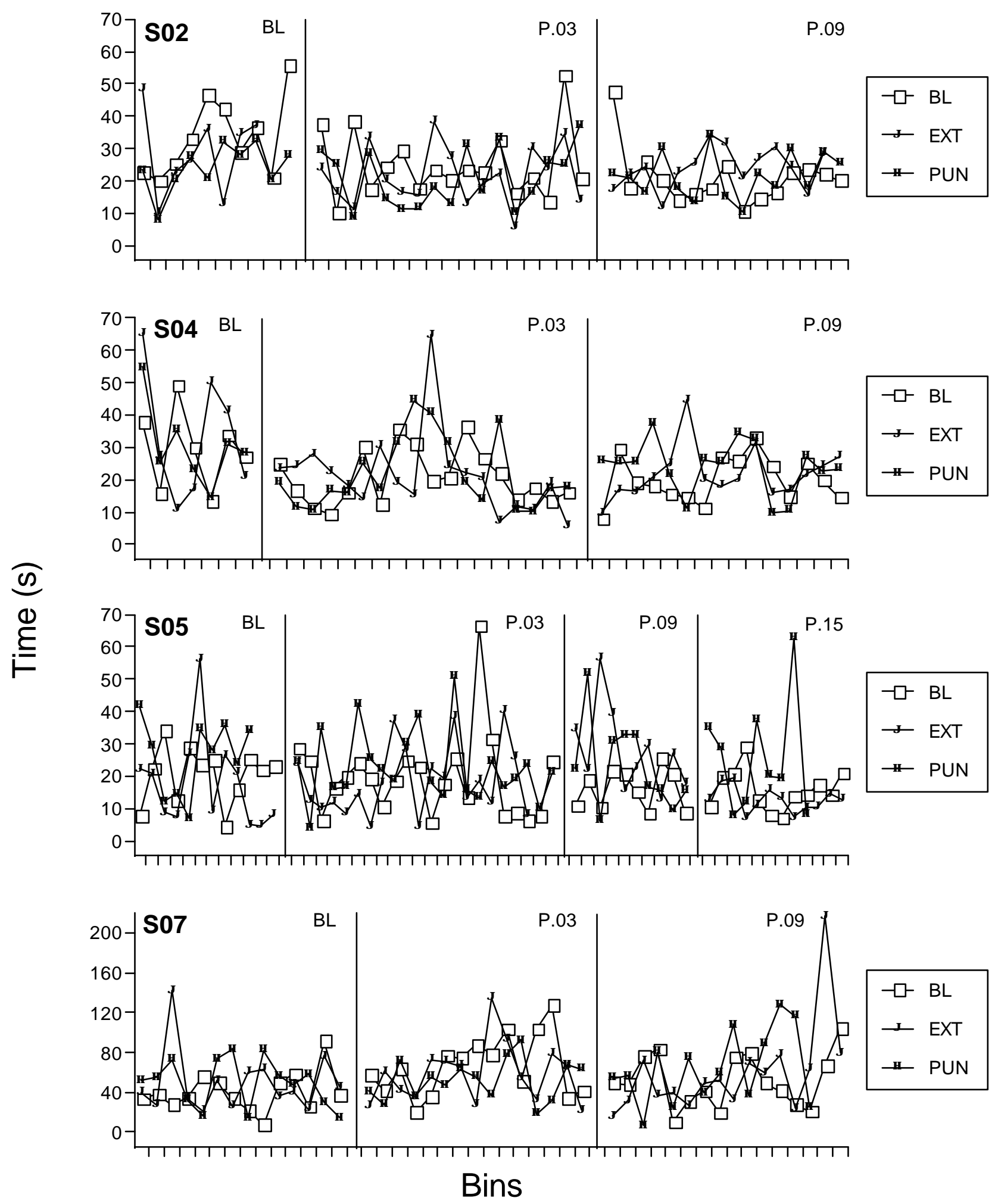

Figure 16. The sum of the time in seconds that subjects spent reviewing the status of their answers and correct answers when an answer was incorrect. The times for ten consecutive frames were summed into bins for each component. P.03 / P.09 / P.15 = experimental phases; $\mathrm{BL}=$ baseline component; EXT = extinction component; $\mathrm{PUN}=$ punishment component. Note differences in the scales of ordinate axes. 
Table 11

$\underline{\text { Statistical Comparison of Time Spent Reviewing Answers }}$

\begin{tabular}{|c|c|c|c|c|c|c|c|}
\hline \multirow[b]{3}{*}{ Subject } & \multirow[b]{3}{*}{ Component } & \multicolumn{6}{|c|}{ Experimental Phase } \\
\hline & & \multicolumn{2}{|c|}{ P.03 } & \multicolumn{2}{|c|}{ P.09 } & \multicolumn{2}{|c|}{ P.15 } \\
\hline & & $\underline{N}$ & $T$ & $\underline{N}$ & $T$ & $\underline{N}$ & $T$ \\
\hline \multirow[t]{2}{*}{ S02 } & EXT & 17 & 57.0 & 14 & 28.0 & & \\
\hline & PUN & 17 & 59.0 & 15 & 49.0 & & \\
\hline \multirow[t]{2}{*}{ S04 } & EXT & 18 & 81.0 & 15 & 52.5 & & \\
\hline & PUN & 19 & 89.5 & 15 & 33.0 & & \\
\hline \multirow[t]{2}{*}{ S05 } & EXT & 20 & 99.0 & 10 & $8.0 *$ & 12 & 23.0 \\
\hline & PUN & 22 & 79.5 & 10 & 12.0 & 9 & 12.0 \\
\hline \multirow[t]{2}{*}{ S07 } & EXT & 15 & 46.0 & 16 & 66.0 & & \\
\hline & PUN & 15 & 41.5 & 14 & 25.0 & & \\
\hline
\end{tabular}

Note. Comparison is of the cumulative amount of time spent reviewing the status of evaluated answers (i.e., correct or incorrect) and examining correct answers. The total time in seconds for ten frames was summed to obtain a single observation. Responding during the extinction and punishment components were compared with responding during the baseline component. P.03 / P.09 / P.15 = experimental phase; $\underline{\mathrm{N}}=$ number of matched pairs of observations; $T=$ value obtained from the Wilcoxon matched-pairs signed-ranks test; EXT = extinction component; PUN $=$ punishment component.

$*_{p}<.05$. 

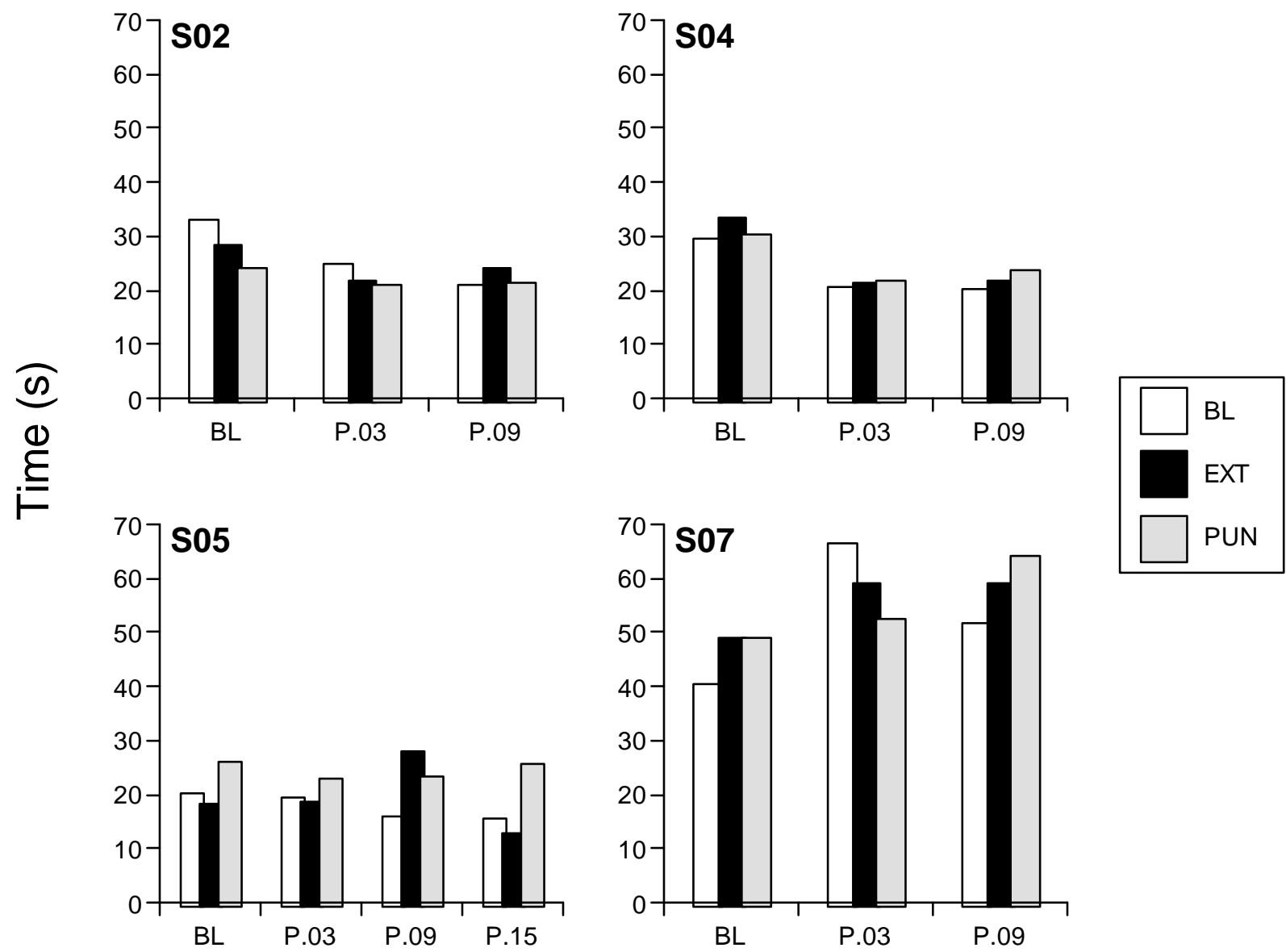

\section{Condition}

Figure 17. The average sums of the time in seconds that subjects spent reviewing the status of their answers and correct answers when an answer was incorrect. The times for ten consecutive frames were summed into bins for each component. Bars indicate the average of those sums. P.03 / P.09 / P.15 = experimental phases; $\mathrm{BL}=$ baseline component EXT $=$ extinction component; PUN = punishment component. 
time spent reviewing answers is consistently longer during the punishment component. $\underline{\text { Off-Task Behavior }}$

The amount of time in seconds that each subject spent playing video games was the operational definition of measurable off-task behavior used throughout the experiment. The total amount of measurable off-task behavior that occurred during the experiment is presented in Figure 18. The total of amount of time per component per session is displayed. The amount of time engaging in measurable off-task behavior was consistent across components, sessions, and subjects. Only S05 and S07 played video games, and they did so for brief durations: the total time for both subjects is close to 3 minutes. Due to the low rates of off-task behavior, no further analyses of these data were conducted.

\section{$\underline{\text { Summary of Results }}$}

Extinction and punishment contingencies for incorrect answers produced variable results. Few, if any, reliable effects were obtained across subjects or experimental phases. Small but unreliable differences were observed for some response measures, possibly suggesting that the response measures used in the present study were differentially sensitive to the effects of the experimental manipulations.

Answer accuracy was not reliably altered by the scheduled extinction and punishment contingencies, as evidenced by the lack of differences in accuracy between components for all subjects. An absence of differences in accuracy between components was apparent through visual inspection of plotted data and was confirmed with statistical analyses. The single statistically significant comparison within these data revealed that the number of correct responses decreased rather than increased while the experimental manipulation was in effect, contrasting with previous findings by other researchers (Costello \& Ferrer, 1976; Munson \& 


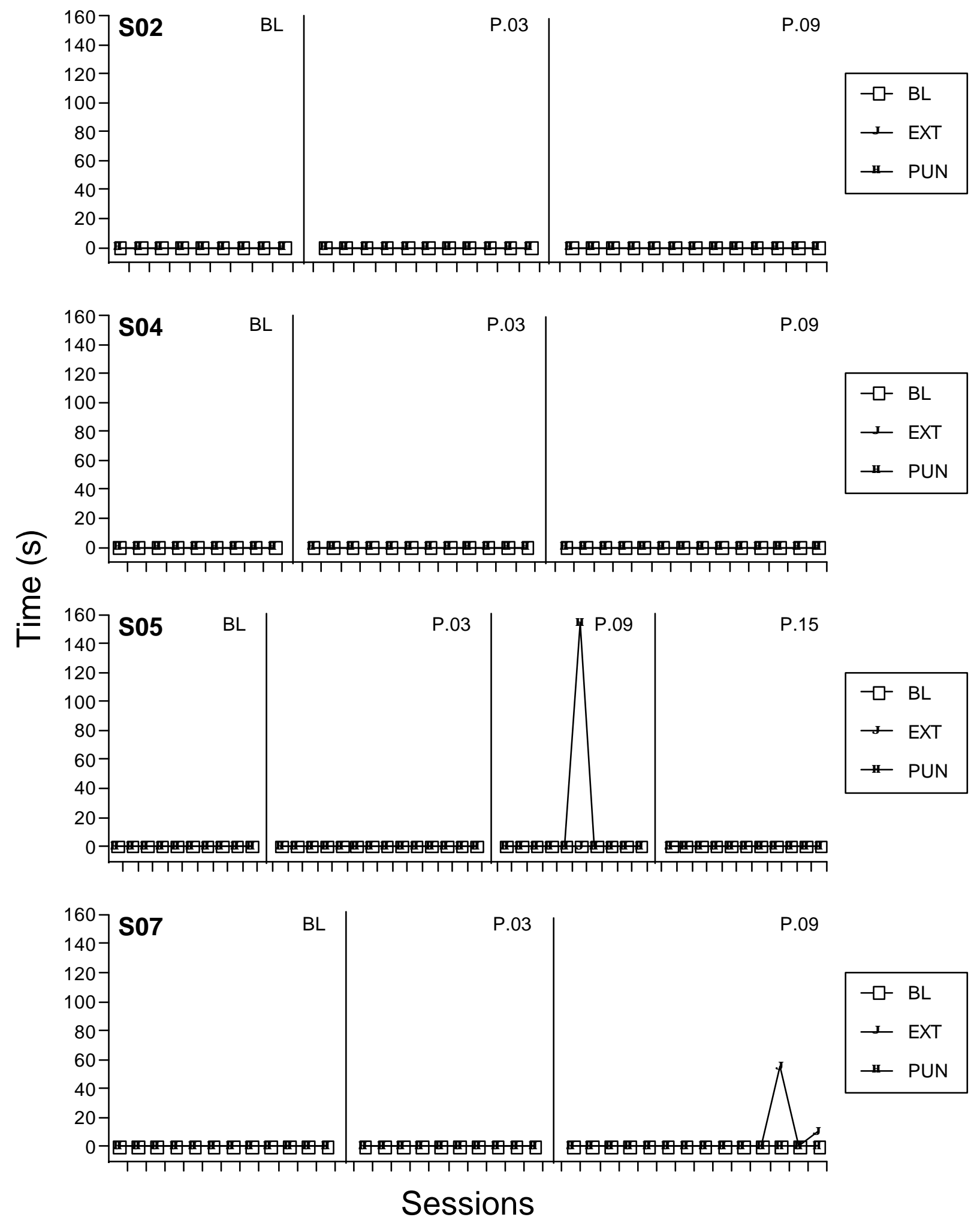

Figure 18. The sum of the time in seconds that subjects played video games. Total times in each component for each session are displayed. P.03 / P.09 / P.15 = experimental phases; $\mathrm{BL}=$ baseline component; $\mathrm{EXT}=$ extinction component; $\mathrm{PUN}=$ punishment component. 
Crosbie, 1998). Similar decreases in answer accuracy were noted for other subjects, where the number of correct answers tended to be higher during the baseline component than the extinction or punishment components.

Examination of overall answer accuracy averages revealed a general trend towards decreasing answer accuracy across experimental phases for all subjects. This trend was detectable through visible inspection of data (see Figures 5 and 7) as well as through statistical summaries (see Table 5). Decreases in answer accuracy corresponded with changes in other response measures that indicated general performance decrements across experimental phases.

An apparent increase in the number of edit keys used across experimental phases occurred for three of four subjects. The increase in the use of edit keys, similar to the general decrease in answer accuracy, did not appear to be systematically related to the the components.

Little variability was found for each subject with respect to the average latency between keystrokes used to construct an answer, and of the differences that were found, none proved to be reliable.

The amount of time that subjects spent viewing correct answers when an answer was incorrect was highly variable, but some trends were noticeable. For two and possibly three subjects there was a tendency to spend more time viewing the correct response during the punishment component relative to both the baseline component and the extinction component. These findings are suggestive, as subjects would be expected to spend more time viewing the correct answer if inaccurate answers are punished with the loss of money.

Composition time was defined as the amount of elapsed time between the appearance of a new frame on the subject's screen and the pressing of the RETURN button the keyboard to submit answers for evaluation. Visual inspection of composition times revealed that these data 
were highly variable and there was little evidence of between-component differences within subjects. Examination of averaged composition times revealed that composition times increased during the first experimental phase relative to the baseline phase. During the first experimental phase, the average composition time during extinction components was longer than the average composition times during punishment components for all subjects. For three of four subjects, the average composition times tended to decrease during later experimental phases relative to the first experimental phase.

The amount of time that passed between the appearance of feedback on the subject's screen and the subject's press of the SPACEBAR button on the keyboard to advance to the next frame was defined as the review time. Visual inspection of data revealed instances of betweencomponent differences for at least two subjects, but these differences were maintained for only one subject during a single experimental phase. Examination of averaged review times revealed that for one subject the length of the review time during the punishment component was higher than the review times during the baseline component. Inconsistent differences were found among other subjects.

Off-task behavior was defined as playing video games, and the duration of time that subjects spent playing video games was assessed across subjects and experimental phases. Two subjects engaged in off-task behavior during the experiment for a total duration of approximately three minutes.

The lack of differences obtained between components and across response measures countered the finding that all subjects accurately identified the component in effect across all phases of the experiment. For three of four subjects, the correct component was identified on nearly ninety percent of trials. Although these values appear high, they were much lower than 
the accuracy values obtained by Madden and Perone (1999) who used a similar componentidentification procedure. Subjects in Madden and Perone's study reliably identified the correct component in over 95 percent of trials, and for one subject only two errors were made throughout the entire study. The accuracy with which subjects identified the correct components in the present study was therefore relatively low, and these data in conjunction with qualitative analyses based on exit interviews (see below) indicated that subjects had some difficulty identifying the components in effect and the corresponding schedule contingencies.

\section{$\underline{\text { Conclusions }}$}

In general, the present results indicated the absence of experimental effects across experimental phases both within and between subjects. Extinction and punishment contingencies did not produce reliable changes in answer accuracy, the number of edit keys used to modify a response, composition time, review time, or the occurrence of off-task behavior. There was some evidence, however, that extinction and punishment contingencies produced differences in the amount of time that subjects spent viewing the correct answer when an answer was incorrect. 


\section{Chapter 5}

\section{Discussion}

Several researchers have recommended the use of mild punishment during programmed instruction to increase students' answer accuracy and decrease their rate of responding (e.g., Holland \& Skinner, 1965). The present experiment was designed to assess this recommendation by comparing answer accuracy and rate of responding as well as the occurrence of off-task behavior in the presence versus the absence of extinction and punishment contingencies for incorrect answers. Analysis of subjects' performance during the present experiment did not support the use of either extinction or mild punishment during programmed instruction. There was little observable change in subjects' responding as a result of the experimental manipulations, and the manipulation of monetary contingencies therefore appeared to be nonfunctional with respect to most response measures. There is therefore a question as to whether the purported extinction and punishment contingencies can be defined as such, for consequences such as extinction and punishment must be defined by their effects on behavior (cf. Azrin \& Holz, 1966). The finding that different monetary consequences did not have consistent effects on subjects' behavior also contrasts with other studies in which the loss of money was a functional punisher during learning tasks (e.g., Brent \& Routh, 1978; Hemry, 1973; Munson \& Crosbie, 1998).

A possible explanation for the lack of results obtained in the present study is that procedural artifacts prevented differential control of responding by component-correlated stimuli and masked the effects of the extinction and punishment contingencies. Punishment and extinction contingencies may therefore have affected subjects' responding, but such changes would not have been detectable with the analyses used in the present study, where changes in responding 
were assessed as a function of changes in the component-correlated stimuli. It is possible that changes in responding occurred only following an incorrect answer and the subjects' direct contact with punishment contingencies (i.e., the appearance of a "subtraction value" next to the incorrect answer). Although changes in component-correlated stimuli were not functional with respect to the response measures used in the present study, it is possible that the appearance of the "subtraction value" was a functional punisher, although this possibility was not directly assessed. Future studies that employ procedures similar to those used in the present study may therefore require methodological changes to ensure that the component-correlated stimuli are more salient than other stimuli that are correlated with the manipulated response contingencies. Once that has been accomplished, it may be possible to provide a comprehensive assessment of the effects of extinction and punishment during programmed instruction.

\section{Interpretation of Results}

Although subjects tended to accurately identify the stimuli correlated with each component of the MULT schedule, the response contingencies correlated with each of the components appeared to be ineffective: reliable between-component differences in answer accuracy, the use of edit keys, latencies between keystrokes, composition times, review times, and off-task behavior were largely absent. This finding contrasts with that of Munson and Crosbie (1998), who used a procedure similar to that of the present study and found that the loss of money for incorrect answers reliably increased answer accuracy and decreased the rate at which subjects progressed through the same program of instruction. Several other researchers (Brent \& Routh, 1978; Hemry, 1973) have also reported that the loss of money for incorrect answers was a functional consequence during learning tasks. Examination of possible alternative sources of control of subjects' responding during the present experiment may provide insight into the 
reasons that the present results conflict with previous findings.

$\underline{\text { Stimulus Control of Responding }}$

Although little evidence of experimental control was found for the response measures used in the present study, differences were found for the amount of time that subjects spent reviewing correct answers when an answer was incorrect. Across experimental phases, the average time that three of four subjects spent reviewing correct answers was higher during the punishment component than during other components. This finding is consistent with previous studies that have found increases in attention to the stimuli relevant to accurate responding as punishment values were increased (e.g., Balaban, Rhodes, \& Neuringer, 1990; Penney, 1967; Stevenson, Weir, \& Zigler, 1959). A possible explanation of the present findings with respect to this response measure is that the appearance of the "subtraction value" (a monetary amount preceded by a negative sign) next to an incorrect answer was the salient stimulus (rather than a color change) that controlled the amount of time spent observing the correct answer. During the baseline and extinction components, nothing appeared next to an incorrect answer. The appearance of a "subtraction value" may therefore have served as a discriminative stimulus for longer viewing times, functioning independently of the component-correlated stimuli. It is therefore possible that the appearance of "subtraction values" was the only functional stimulus correlated with punishment for incorrect answers, and subjects' responding was controlled by this stimulus rather than by the component-correlated stimuli.

\section{$\underline{\text { Identifying Component Colors }}$}

On average, S05 correctly identified the color of the component in effect on $95 \%$ of all possible attempts, with subject S05 obtaining an overall accuracy of 92\% and S07 obtaining an overall accuracy of $91 \%$. Subject S04 obtained a much lower accuracy than the other subjects, 
with an overall average correct of $74 \%$. Relative to both nonhuman and human experimental research that has used a similar "component identification" procedure, all of the averages obtained in the present study were lower than expected. For example, Madden and Perone (1999) investigated the extent to which subjects maximized the amount of reinforcement obtained on an operant task. The task involved responding (clicking on a small moving target with a computer mouse) during one of two concurrently available schedules of reinforcement. One of the two schedules had a higher "payoff rate" (i.e., a denser schedule of reinforcement) than the other, and subjects could switch between the schedules. The experimental procedure was designed to assess the conditions under which subjects would reliably switch to the schedule that produced a higher amount of reinforcement for the same task. When the experimental procedure required the subjects to select a stimulus of the same color as the color that was correlated with each schedule (similar to the component-identification procedure in the present study), subjects began to reliably switch to the schedule with the higher "payoff rate." This finding indicated that in the process of identifying the color correlated with each of the schedules, subjects also began to discriminate the "payoff" differences between the schedules. Although the procedure used by Madden and Perone for identifying the schedule-correlated color was similar to the procedure used in the present study, Madden and Perone's subjects were more accurate at the identification task: two of their subjects correctly identified the schedulecorrelated stimuli on over $99 \%$ of trials and a third subject was accurate on $96 \%$ of trials.

There are several possible reasons that subjects in the present study were less accurate at identifying the color of the component in effect than were subjects in previous studies. One possible reason is related to the complexity of the task. In the Madden and Perone (1999) study, the subjects were required to engage in the rather simple task of clicking on a moving target that 
appeared on the screen. The task of subjects in the present study involved numerous activities, including reading a frame of instructional material, composing and editing answers, and examining the status of answers (i.e., correct or incorrect). The complexity of the task in the present study may have made it more difficult for subjects to attend to the component-correlated stimuli. Examination of Figure 1 reveals another important characteristic of the present task that may have contributed to the relatively poor component-identification accuracies that were obtained. Components of the MULT schedule were indicated by thin bands of color that bounded the top and bottom of each numbered indicator. By comparison, when subjects in the Madden and Perone study clicked on a button labeled "LOOK," one half of the subject's entire screen changed to the color correlated with the schedule that was in effect. The bands of color that bounded the numbered indicators in the present study represented only a very small portion of the screen by comparison and changes in those colors may have been more difficult to detect for that reason. In summary, the complexity of the current task and the salience of the stimuli correlated with the schedule components may therefore have contributed to the lower accuracies obtained in component identification procedure.

\section{$\underline{\text { Off-Task Behavior }}$}

Very little off-task behavior occurred at any time and for any subject throughout the experiment. This finding is consistent with the lack of differential control of responding by component-correlated stimuli. Subjects would not be expected to play video games as a means of escaping from the extinction or punishment contingencies if subjects were unaware of when extinction and punishment contingencies were in effect, thereby obviating the possibility for a functional escape response. It is also likely that a significant reinforcer for subjects, especially towards the end of the experiment, was escape from the task altogether: playing games would 
have prolonged the task and therefore may not have been seen as a means for escape. Under these conditions, the reliable occurrence of off-task behavior would be difficult to establish. $\underline{\text { Verbal Reports }}$

Evidence that subjects failed to discriminate the different contingencies correlated with each component also comes from subjects' verbal reports obtained at the end of the experiment. Upon completion of the experiment, each subject was asked to describe critical features of the experiment, including the function of the different component colors. None of the subjects could accurately describe the contingencies that were unique to each of the components. Subjects could, however, describe differences in response consequences during the experimental phase relative to the baseline phase (e.g., "At some point during the experiment I started to lose money when I answered incorrectly...”). Human subjects do not necessarily need be able to describe a contingency for it to have a measurable and reliable effect on their behavior (Greenspoon \& Brownstein, 1967), and the inability of subjects in the present study to accurately describe the experimental contingencies is not a sufficient reason for the observed lack of experimental control. However, subjects' verbal reports at the conclusion of the experiment corresponded with the observation that experimental control by response contingencies had not been established by changes in the colors of the component indicators.

\section{General Performance Decrements}

In general, answer accuracy decreased across experimental phases for all subjects. This decrease in answer accuracy was less than that reported by Munson and Crosbie (1998), who used the same program of instruction and a similar set of procedures. These researchers found large decreases in answer accuracy for two of three subjects across sessions when a punishment contingency was not in effect (Figure 1, p. 239). It is therefore possible that the programmed 
extinction and punishment contingencies used in the present study were functional, serving to prevent similar but larger performance decrements than would have otherwise been observed. This result could have been masked by the lack of between-component differences and decrements that would otherwise have been isolated to the baseline component.

\section{$\underline{\text { Summary of Interpretation }}$}

There are at least two possible interpretations of the results from the present experiment. One interpretation is that the purported extinction and punishment contingencies were nonfunctional. Another interpretation is that the purported extinction and punishment contingencies did have an effect on answer accuracy, but those effects were not controlled by the presence or absence of the component-correlated stimuli. Instead, the effects of extinction and punishment may have been effective in increasing answer accuracy across all components, as subjects failed to come under differential control of the component-correlated stimuli. It is also possible that extinction and punishment contingencies prevented larger decreases in answer accuracy than otherwise would have occurred. These conjectures are speculative, but there is evidence that the loss of money for incorrect answers was a functional punisher. This evidence comes from the longer amounts of time that subjects spent observing correct answers when an answer was evaluated as incorrect. It is possible that this effect, though small and not statistically reliable, was detectable through visual inspection due to the control of observing responses by the presence of "subtraction values" that appeared next to incorrect answers. These "subtraction values" may have functioned as discriminative stimuli for avoiding an incorrect answer on later cycles through a set, i.e., longer observing times were likely to have been reinforced by avoidance of an incorrect answer to the same frame and the loss of more money. 


\section{$\underline{\text { Future Studies }}$}

The present study did not demonstrate experimental control over subject responding to the degree that was originally anticipated. It is likely that the reasons for this were procedural. Future studies using a similar methodology should consider changes to the present procedures prior to implementation. These following suggested changes would likely simplify data analysis and interpretation as well as facilitate the control of responding by component-correlated stimuli.

\section{Component Length}

The parameters of the MULT schedule used in the present study may have contributed to the ineffectiveness of the component-correlated stimuli in establishing control of subjects' responding. Each component of the MULT schedule was in effect for six minutes and was then alternated. By comparison, Munson and Crosbie (1998) used a MULT schedule with each component lasting for an entire set of frames. The much more rapid alternation of components in the present study relative to Munson and Crosbie's may have prevented a sustained and necessary contact with the contingencies associated with each component. The brevity of the components in the present study is an important feature of the MULT schedule because subjects only came into contact with the contingencies in effect during a component following an incorrect answer. If few incorrect answers were made (for example, by S05), there would be little opportunity to establish control of responding by component-specific contingencies. An increase in the length of each component and a corresponding increase in the exposure to the contingencies within a component might have facilitated stimulus control and should be considered for future studies.

\section{$\underline{\text { Number of Components }}$}

In addition to increasing the length of each component in the MULT schedule, the number of 
components should be reduced from three to two. This could be most efficiently accomplished by removing the extinction component used in the present study. With only two components in effect (i.e., baseline and punishment), the changes in response contingencies and the correlation of those changes with the component-correlated stimuli would be easier to detect.

\section{$\underline{\text { Return to Baseline Contingencies }}$}

In the present analysis, it was speculated that the extinction and punishment contingencies may have prevented a greater decrease in answer accuracy and increases in response rates than may otherwise have occurred. This conjecture could have been assessed by including a final experimental phase for all subjects where the extinction and punishment contingencies were removed and the baseline phase was reinstated (i.e., a "return to baseline"). If large drops in accuracy and increases in response rates were observed following a return to the baseline contingencies, evidence would have been available for describing the purported extinction and punishment contingencies as functional response consequences. A return to baseline contingencies in future studies using similar methodologies may be necessary for similar reasons.

\section{$\underline{\text { Session Length }}$}

The present study defined the length of a session as equivalent to the length of a set from the program of instruction. This definition introduced complications in data analysis and interpretation. Matched-pairs necessary for nonparametric analyses couldn't be obtained for some sets as subjects invariably completed a session with variable number of answers within each component; sometimes the number of answers within a component was zero. The solution to this problem was to group data into bins for analysis. Nonparametric analyses were then conducted by matching "pairs" of bins, e.g., the fifth bin in the extinction component with the 
fifth bin of the baseline component. However, a more proactive solution would be to fix the length of a session to include equivalent numbers of responses within each component. Data analysis and interpretation would be greatly simplified if equivalent numbers of responses occurred within each component as a criterion for the subject's progression through the program.

\section{$\underline{\text { Subject Payments }}$}

Analysis and interpretation of results in the present study was complicated by increases in payments to subjects for correct responses following the subject's transition from the baseline phase to the experimental phases. Changes in responding that occurred following a transition to the experimental phases could then be attributed to increases in the value of correct answers rather than the introduction of the extinction and punishment contingencies alone. Future studies using similar procedures could avoid this problem by setting the value of each correct response at a higher level during baseline phases so that it would not need to be altered during later phases. The value of the correct response should be high enough that the introduction of punishment contingencies would not reduce overall earnings below those values that were advertised during subject recruitment. For example, in the present study, the value of each correct response could have been set at $\$ .09$ without necessitating a change during later experimental phases.

\section{$\underline{\text { Conclusions }}$}

Additional experimentation with the above mentioned procedural controls would facilitate an analysis of the effects of extinction and punishment during programmed instruction.

Conclusions about the effects of reinforcement, extinction, and punishment on responding during programmed instruction based on the present data must, for the most part, remain at the level of conjecture. Some suggestive results were obtained (i.e., time spent reviewing correct answers), 
but the reasons for this are unclear. Only with additional experimentation will procedures be identified that will allow researchers to reliably obtain experimental control of subjects' responding. Once control of subject responding during programmed instruction is reliably brought under the control of variable response contingencies, the effects of extinction and punishment on subjects' answer accuracy and rate of responding can be delineated. Controlled studies of this nature are especially important because of the failure of previous researchers (e.g., Munson \& Crosbie, 1998) to assess for the presence of unwanted side-effects of punishment during programmed instruction. 


\section{References}

Anderson, R. C., Kulhavy, R. W., \& Andre, T. (1971). Feedback procedures in programmed instruction. Journal of Educational Psychology, 62, 148-156.

Angell, G. W. (1949). The effect of immediate knowledge of quiz results and final examination scores in freshman chemistry. Journal of Educational Research, 42, 391-394.

Azrin, N. H., \& Holz, W. C. (1966). Punishment. In W. K. Honig (Ed.) Operant behavior: Areas of research and application (pp. 380-447). Englewood Cliffs, NJ: Prentice Hall.

Balaban, M. T., Rhodes, D. L., \& Neuringer, A. (1990). Orienting and defense responses to punishment: Effects on learning. Biological Psychology, 30, 203-217.

Borresen, C. R. (1973). Reward, punishment, and reversal on a mirror-tracing task. Perceptual and Motor Skills, 37, 199-202.

Brackbill, Y., \& O’Hara, J. (1958). The relative effectiveness of reward and punishment for discrimination learning in children. Journal of Comparative and Physiological Psychology, 51, 747-751.

Brent, D. E., \& Routh, D. K. (1978). Response cost and impulsive word recognition errors in reading-disabled children. Journal of Abnormal Child Psychology, 6, 211-219.

Carr, E. G., Newson, C. D., \& Binkoff, J. A. (1980). Escape as a factor in the aggressive behavior of two retarded children. Journal of Applied Behavior Analysis, 13, 113-129.

Cook, D., \& Mechner, F. (1962). Fundamentals of programed instruction. In S. Margulies \& L. D. Eigen (Eds.), Applied programed instruction (pp. 2-14).

Costello, J., \& Ferrer, J. (1976). Punishment contingencies for the reduction of incorrect responses during articulation instruction. Journal of Communication Disorders, 9, 43-61.

Coulson, J. E., \& Silberman, H. F. (1960). Effects of three variables in a teaching machine. Journal of Educational Psychology, 51, 135-143.

Crosbie, J., \& Kelly, G. (1994). Effects of imposed postfeedback delays in programmed instruction. Journal of Applied Behavior Analysis, 27, 483-491.

Cunningham, S. J., \& Knights, R. M. (1978). The performance of hyperactive and normal boys under differing reward and punishment schedules. Journal of Pediatric Psychology, 3 , 195-201. 
Donahue, B., \& Ratliff, R. G. (1976). The effects of reward, punishment, and knowledge of results on children's discrimination learning. Journal of Genetic Psychology, 129, 97103.

Edginton, E. S. (1982). Nonparametric tests for single-subject multiple schedule experiments. Behavioral Assessment, 4, 83-91.

Erickson, J. R. (1970). Effects of punishment for errors on discrimination learning by humans. Journal of Experimental Psychology, 83, 112-119.

Evans, J. L., Glaser, R., \& Homme, L. E. (1962). An investigation of 'teaching machine' variables using learning programs in symbolic logic. Journal of Educational Research, $\underline{55,433-452 .}$

Fago, G. \& Fowler, H. (1972). Facilitated discrimination learning as effected by responsecontingent neutral and aversive stimuli. Learning and Motivation, 3, 20-30.

Firestone, P., \& Douglas, V. I. (1977). The effects of verbal and material rewards and punishers on the performance of impulsive and reflective children. Child Study Journal, 7, 71-78.

Fowler, H., Hochhauser, M., \& Wischner, G. J. (1981). Facilitating stimulus effects of reward and punishment in discrimination learning. Animal Learning and Behavior, 9, 16-20.

Fowler, H., Spelt, P. F., \& Wischner, G. J. (1967). Discrimination performance as affected by training procedure, problem difficulty, and shock for the correct response. Journal of Experimental Psychology, 75, 432-436.

Foxx, R. M. (1984). The use of a negative reinforcement procedure to increase the performance of autistic and mentally retarded children on discrimination training tasks. Analysis and Intervention in Developmental Disabilities, 4, 253-265.

Getsie, R. L., Langer, P., \& Glass, G. V. (1985). Meta-analysis of the effects of type and combination of feedback on children's discrimination learning. Review of Educational Research, 55, 9-22.

Greenspoon, J., \& Brownstein, A. J. (1967). Awareness in verbal conditioning. Journal of Experimental Research in Personality, 2, 295-308.

Harris, L. M., \& Tramontana, J. (1973). Discrimination learning of retarded children as a function of positive reinforcement and response cost. American Journal of Mental Deficiency, 78, 216-219.

Hemry, F. P. (1973). Effect of reinforcement conditions on a discrimination learning task for impulsive versus reflective children. Child Development, 44, 657-660. 
Heron, T. E. (1978). Punishment: A review of the literature with implications for the teacher of mainstreamed children. The Journal of Special Education, 12, 243-252.

Hoge, M. A., \& Stocking, R. J. (1912). A note on the relative value of punishment and reward as motives. Journal of Animal Behavior, 2, 43-50.

Holland, J. G. (1960). Teaching machines: An application of principles from the laboratory. In A. A. Lumsdaine \& R. Glaser (Eds.), Teaching machines and programmed learning (pp. 215-228).

Holland, J. G. (1961). Evaluating teaching machines and programs. Teachers College Record, 63, 56-65.

Holland, J. G., \& Skinner, B. F. (1961). The Analysis of Behavior: A Program for SelfInstruction. New York: McGraw-Hill.

Kaess, W., \& Zeaman, D. (1960). Positive and negative knowledge of results on a Pressy-type punchboard. Journal of Experimental Psychology, 60, 12-17.

Kelly, G. \& Crosbie, J. (1997). Immediate and delayed effects of imposed postfeedback delays in computerized programmed instruction. The Psychological Record, 47, 687-698.

Kodera, T. L., \& Rilling, M. (1976). Procedural antecedents of behavioral contrast: A reexamination of errorless learning. Journal of the Experimental Analysis of Behavior, 25, $27-42$.

Kulik, J. A., Cohen, P. A., \& Ebeling, B. J. (1980). Effectiveness of programmed instruction in higher education: A meta-analysis of findings. Educational Evaluation and Policy Analysis, 2, 51-64.

Madden, G. J. (1995). Effects of observing schedule-correlated stimuli and reinforcers on human sensitivity to concurrent variable-interval schedules of reinforcement. Unpublished Doctoral Dissertation. Morgantown, WV.

Madden, G. J., \& Perone, M. (1999). Human sensitivity to concurrent schedules of reinforcement: Effects of observing schedule-correlated stimuli. Journal of the Experimental Analysis of Behavior,71, 303-318.

Markle, S. M. (1967). Empirical testing of programs. In P. C. Lange (Ed.), Programmed Instruction (pp. 104-138). Chicago: University of Chicago Press.

Melaragno, R. J. (1960). Effect of negative reinforcement in an automated teaching setting. Psychological Reports, 7, 381-384. 
Merbaum, M. (1973). The modification of self-destructive behavior by a mother-therapist using aversive stimulation. Behavior Therapy, 4, 442-447.

Meyer, S. R. (1960). Report on the initial test of a junior high school vocabulary program. In A. A. Lumsdaine \& R Glaser (Eds.), Teaching Machines and Programmed Learning (pp. 229-246). Washington, D. C.: National Education Association.

Michael, J. (1974). Statistical inference for individual organism research: Mixed blessing or curse? Journal of Applied Behavior Analysis, 7, 647-653.

Miller, F. D., Moffat, G. H., Cotter. P. D., \& Ochocki, T. E. (1973). Verbal reinforcement conditions, task complexity, age and sex in children's discrimination learning. Perceptual and Motor Skills, 37, 531-534.

Moses, J. A., Ratliff, R. G., \& Ratliff, A. R. (1979). Discrimination learning of delinquent boys as a function of reinforcement contingency and delinquency subtype. Journal of Abnormal Child Psychology, 7, 443-453.

Mueller, D. J., \& Gumina, J. M. (1972). Methodological studies involving reward and punishment in human learning: I. Effects of design and instructions. Perceptual and Motor Skills, 34, 63-74.

Muenzinger, K. F. (1934). Motivation in learning: I. Electric shock for correct response on the visual discrimination habit. Journal of Comparative Psychology, 17, 267-277.

Muenzinger, K. F. (1938). Vicarious trial and error at a point of choice: I. A general survey of its relation to learning efficiency. Journal of Genetic Psychology, 53, 75-86.

Munson, K. J., \& Crosbie, J. (1998). Effects of response cost in computerized programmed instruction. The Psychological Record, 48, 233-250.

Newsom, C., Favell, J. E., \& Rincover, A. (1983). The side effects of punishment. In S. Axelrod \& J. Apsche (Eds.), The effects of punishment on human behavior (pp. 285-316).

Ochocki, T. E., Cotter, P. D., \& Miller, F. D. (1974). Verbal reinforcement combinations, task complexity, and overtraining on discrimination reversal learning in children. Journal of Genetic Psychology, 90, 213-219.

O'Leary, K. D., \& Becker, W. C. (1969). The effects of the intensity of a teacher's reprimands on children's behavior. Journal of School Psychology, 7, 8-11.

Penny, R. K. (1967). Effect of reward and punishment and children's orientation and discrimination learning. Journal of Experimental Psychology, 75, 140-142. 
Penny, R. K., \& Lupton, A. A. (1961). Children's discrimination learning as a function of reward and punishment. Journal of Comparative and Physiological Psychology, 54, 449451.

Perone, M. (1990). Avoiding aversive control: A review of "Coercion and its Fallout" by Murray Sidman. Experimental Analysis of Human Behavior Bulletin, 8, 16-20.

Ratliff, R. G., \& Root, J. R. (1974). Two-choice discrimination learning in children as a joint function of incentive level and punishment. Journal of Genetic Psychology, 124, 249257.

Reuter, J. H., \& Chung, H. S. (1988). The contribution of the rewarded and the nonrewarded stimulus in visual discrimination learning in mice. Behavioural Brain Research, 27, 269272.

Schusterman, R. J. (1966). Serial discrimination - reversal learning with and without errors by the California sea lion. Journal of the Experimental Analysis of Behavior, 9, 593-600.

Sidman, M. (1960). Tactics of Scientific Research. New York: Basic Books.

Sidman, M., \& Stoddard, L. T. (1967). The effectiveness of fading in programming a simultaneous form discrimination for retarded children. Journal of the Experimental Analysis of Behavior, 10, 3-15.

Siegel, S. (1956). Nonparametric Statistics for the Behavioral Sciences. Tokyo: McGraw-Hill Kogakusha.

Skinner, B. F. (1953). Science and Human Behavior. New York: The Macmillan Company.

Skinner, B. F. (1986, October). Programmed instruction revisited. Phi Delta Kappan, 68, 103110.

Skinner, B. F., \& Ferster, C. B. (1957). Schedules of Reinforcement. New York: Prentice-Hall, Inc.

Skinner, B. F., \& Holland, J. G. (1965). The use of teaching machines in college instruction. In R. Glaser (Ed.), Teaching machines and programed learning, II (pp. 159-172). Washington, D. C.: National Education Association of the United States.

Stevenson, H. W., Weir, M. W., \& Zigler, E. F. (1959). Discrimination learning in children as a function of motive-incentive conditions. Psychological Reports, 5, 95-98. 
Stoddard, L. T., \& Sidman, M. (1967). The effects of errors on children's performance on a circle-ellipse discrimination. Journal of the Experimental Analysis of Behavior, 10, 261270.

Taylor, G. T. (1974). Varied functions of punishment in differential instrumental conditioning. Journal of Experimental Psychology, 102, 298-307.

Terrace, H. S. (1963). Discrimination learning with and without "errors." Journal of the Experimental Analysis of Behavior, 6, 1-27.

Thomas, D. R., Becker, W. C., \& Armstrong, M. (1968). Production and elimination of disruptive classroom behavior by systematically varying teacher's behavior. Journal of Applied Behavior Analysis, 1, 35-45.

Tindall, R. C., \& Ratliff, R. G. (1974). Interaction of reinforcement conditions and developmental level in a two-choice discrimination task with children. $\underline{\text { Journal of }}$ Experimental Child Psychology, 18, 183-189.

Trent, J. T. (1983). Role of reinforcement and response cost in discrimination learning. Psychological Reports, 53, 207-211.

Warden, C. J., \& Aylesworth, M. (1927). The relative value of reward and punishment in the formation of a visual discrimination habit in the white rat. Journal of Comparative Psychology, 7, 117-127.

Wischner, G. J., Fowler, H., \& Kushnick, S. A. (1963). Effect of strength of punishment for "Correct" or "Incorrect" responses on visual discrimination performance. Journal of Experimental Psychology, 65, 131-138.

Witte, K. L., \& Johnson, R. K. (1973). Children's discrimination learning as related to delayed punishment. Bulletin of the Psychonomic Society, 2, 146-148.

Wright, J. C., \& Smothergill, D. (1967). Observing behavior and children's discrimination learning under delayed reinforcement. Journal of Experimental Child Psychology, 5, $430-440$. 


\section{Appendix A}

\section{Pre-Experimental Questionnaire}

\section{Human Learning and Motivation}

This questionnaire is for individuals who are interested in participating in a research project entitled Human Learning and Motivation. With this questionnaire I am attempting to identify WVU students who are 18 years or older and qualify for participation in the study. If you qualify for the study, you may be asked to come to my laboratory approximately five times per week for several weeks to work. The money that you earn at the job will be based on your performance. The investigators for the research project are Mr. Stephen C. Scherer and Dr. Julie S. Vargas of the Educational Psychology Program. The research project is being conducted in partial fulfillment of the requirements of the Doctoral Program in Educational Psychology.

Your decision to answer this questionnaire is voluntary. You do not have to answer every question. Your decision to answer the questionnaire will not affect your class standing or grades, or your status on an athletic team. By filling out this form, you are only expressing interest in the project; you are not committing yourself to participating (and I am not promising that you will be invited to participate).

I will keep your responses to the questionnaire confidential. I will not release your name or phone number to other investigators. I am asking for them only so that I can contact you if I am able to offer you an opportunity to participate in the study on learning and motivation.

Instructions. If you are interested in participating in the research project entitled Human Learning and Motivation, please fill out this survey and return it to Stephen C. Scherer, Allen Hall Room 507-E. Stephen will contact you by telephone if he is able to offer you the opportunity of participating.

Name Age Gender Telephone No.

1. Please list all psychology classes completed or in progress.

2. Have you ever participated in a psychology experiment?

If you answered "yes," briefly describe the experiment(s):

For the remaining questions, circle the response that best describes you:

3. I live: (a) at home with my parents. (b) in an on-campus dormitory. (c) in a fraternity/sorority house. (d) in an apartment or house that I rent or own. (e) Other (specify below)

4. My living expenses (rent, food, entertainment, etc.): (a) are paid in full by my parents. (b) are partially paid by my parents, but I pay the rest. (c) are partially paid by scholarships, but I pay the rest. (d) are paid in full by myself. (e) Other (specify below) 
5. During an average week, I eat: (a) all of my meals at home or in the dorm. (b) 1 or 2 lunches or dinners at a restaurant (can include anything from the Mountain Lair to an expensive restaurant). (c) 3 or 4 lunches or dinners at a restaurant. (d) almost all of my meals at restaurants. (e) Other (specify below)

6. When I eat out, I usually spend: (a) two dollars or less. (b) between two and four dollars. (c) between four and seven dollars. (d) more than seven dollars.

7. From day-to-day: (a) I never have enough money for simple items like snacks, drinks, etc. (b) I sometimes have enough money for such simple items, but there are days when I am broke. (c) I usually have enough pocket money for such simple items. (d) I always have enough money to buy a Coke or a snack if I want one.

8. One the weekends: (a) I never have enough money to go out and have a good time. (b) I sometimes have enough money to go out, but sometimes I have to stay home. (c) I usually have enough money to go out and have a good time. (d) I always have enough money to do what I want.

9. When I go out at night, I: (a) choose an activity that doesn't cost any money. (b) usually spend $\$ 5.00$ or less. (c) usually spend $\$ 10.00$ or less. (d) usually spend more than $\$ 10.00$.

10. I work: (a) full-time (20-40 hours per week). (b) part-time (5-20 hours per week). (c) I don't have a job, but am looking for one. (d) I don't have a job and am not looking for one.

11. If I had more money I would:

a. spend it on

b. save it for

12. In an average week, I could work hours on the project entitled Human Learning and Motivation. (a) less than 5 (b) between 6 and 10 (c) between 11 and 20 (d) more than 21 hours 


\section{Appendix B}

Consent and Information Form

\section{Introduction}

I, have been asked to participate in this research study which has been explained to me by Stephen C. Scherer. This study is being conducted in partial fulfillment of the requirements for Mr. Scherer's doctoral dissertation in the College of Human Resources and Education at West Virginia University.

\section{Purposes}

I understand that the purpose of this study is examine how people learn while using a computerassisted instructional program. Approximately four to six people will participate in this project.

\section{Procedures}

I understand that I will always work in Room 507-E Allen Hall at West Virginia University. Participation in this study will require that I work here alone for two sessions lasting about 30 minutes each with short breaks between sessions. I will be allowed to leave the room when I take breaks between the sessions, but during a session I must remain in the room. In the event of an emergency, I understand that I am free to leave at any time--if I become ill, for example. I know that the investigator will always be next door while I am working.

I understand that I will be working with a computer. During each session the computer will present a series of sentences that are missing one or more words. My task is to use the keyboard to complete each sentence by typing in the missing word(s). The computer will then evaluate my response(s) and provide me with feedback. I understand that I am not to use the computer for any other purpose besides working on the activities demonstrated, and that I am not to disturb this equipment in any way, for example by opening disk drives or restarting the computer. My performance when using this equipment will be monitored both visually and electronically.

It is essential to the success of this project that I work under standard conditions from day to day. Therefore, personal items such as watches, reading or writing materials, any sort of tool, portable radios, tape players, CD players, food, drink, or smoking materials are not allowed in the workroom. If I bring any of these items, the investigator will keep them for me while I work. To minimize distractions while I work, the door and window shade must remain shut and I must wear headphones that connect to the computer's sound system. Soft "white noise" will be generated from a radio in the workroom to mask outside noises. The researcher will adjust the white noise to a level that I find comfortable.

To prevent my experience and opinions from influencing other students who may participate, I agree not to discuss the research with anyone. This is necessary because other students may join this project during or after my participation, and I understand that they need to enter the project 
without any preconceived ideas about it.

I understand that the actual length of my participation cannot be determined before the project begins because it will depend on the day-to-day results. However, I am promised that the project will last at least 20 hours and not more than 40 hours.

I understand that my schedule must meet the following requirements: (a) I must complete 5 to 10 hours of work per week, scheduled 5 to 7 days per week at times convenient for me and the investigator. (b) I must follow the schedule I set up (come to all sessions and come on time). (c) If an absence is unavoidable, I must notify the investigator as soon as I know about it. I know that the investigator's phone number is in the "Contact Persons" section of this consent form. I understand that this project requires consistent attendance; if I miss a session or come late it creates serious problems for the investigator. If unexcused absences or tardiness become a persistent problem, or if I am unable to meet my work schedule, my participation in the project may be terminated.

I understand that the rules concerning personal items, the door, the window shade and the headphones are important and I should take care to follow these rules. Should I break these rules, the records of my session will be invalid and my participation in the project may be terminated.

\section{Benefits}

I understand that this study is not expected to be of direct benefit to me, but the knowledge gained may be of benefit to others. I understand that I will be paid for participating in this study.

\section{Risks and Discomforts}

I understand that my participation in this study poses no foreseeable risks to my health or psychological well being, except perhaps for the mild frustration that many people experience when learning something new.

\section{Financial Considerations}

I understand that I will not be expected to pay any costs or fees as a result of my participation in this study.

I understand that I can earn as much as $\$ 117.05$ over the course of the project. Because my pay will depend on my performance, however, I am not guaranteed to earn any particular amount. I understand that payment will be made at the end of my participation in the project. The investigator will keep careful records of my earnings and I will be given a written statement of my earnings every day.

I understand the importance of my participation for the full duration of the project; substantial time and money is lost when someone quits the project early. There is a bonus to encourage me to work for the entire project. If I complete the entire project, I will be awarded a bonus of approximately $\$ 0.60$ per session. This bonus will be in addition to my regular earnings during 
the sessions. Considering both the bonus plan and my session earnings, I could earn as much as $\$ 146.45$ total, depending on my performance and the duration of the project.

If I fail to complete the project for any reason, I understand that I will receive my session earnings, but I will forfeit the bonus.

\section{Contact Persons}

The principal investigator in this project is Stephen C. Scherer, a doctoral student in the WVU of the Educational Psychology Program (office: Allen Hall, Room 513-B, telephone: 365-8276). The project is being supervised by Julie S. Vargas, a professor in the Department (office: 506-E Allen Hall, telephone: 293-2525 ext. 1365 or 340-776-6743 from June 15 to August 15). I should contact Mr. Scherer or Dr. Vargas if questions arise in connection with my participation in the research, including such matters as schedule problems (e.g., unanticipated absence or tardiness).

If I have questions about my rights as a research subject, I understand that I may contact the Executive Secretary of the Institutional Review Board at 293-7073.

\section{Confidentiality}

I understand that any information about me obtained as a result of my participation in this research will be kept as confidential as legally possible. I understand also that my research records, just like hospital records, may be subpoenaed by court order or may be inspected by federal regulatory authorities. However, I also understand that the investigators will take every legal step to protect my privacy. In any publications that result from this research, neither my name nor any information from which I might be identified will be published without my consent.

\section{Voluntary Participation}

I understand that my participation in this project is voluntary. I am free to quit at any time, and if I do so I will be paid all of the money that I have earned during the sessions I have completed. I understand that refusal to participate or withdrawal from the project will involve no penalty or loss of benefits and will not reflect on my grades, status on an athletic team, or class standing. I understand, however, that if I do quit I will forfeit the bonus.

Before signing this consent form, I participated in a sample session designed to familiarize me with the operation of the computer program and give me a better idea of what the project will be like. I have been informed that I will be paid $\$ 5.00$ for my time and effort in the sample session, regardless of whether I decide to participate any further.

I have been given the opportunity to ask questions about this research project, and I have received answers concerning areas that I did not understand.

Upon signing this form, I will receive a copy. 
I willingly consent to participate in this research.

\begin{tabular}{|c|c|c|c|}
\hline \multirow[t]{2}{*}{ Participant } & \multirow[b]{2}{*}{ (Printed Name) } & \multirow[b]{2}{*}{ (Signed Name) } & \multirow[b]{2}{*}{$\overline{\text { (Date and Time) }}$} \\
\hline & & & \\
\hline \multirow[t]{2}{*}{ Investigator } & & & \\
\hline & (Printed Name) & (Signed Name) & $\overline{\text { (Date and Time) }}$ \\
\hline
\end{tabular}


Appendix C

Help File

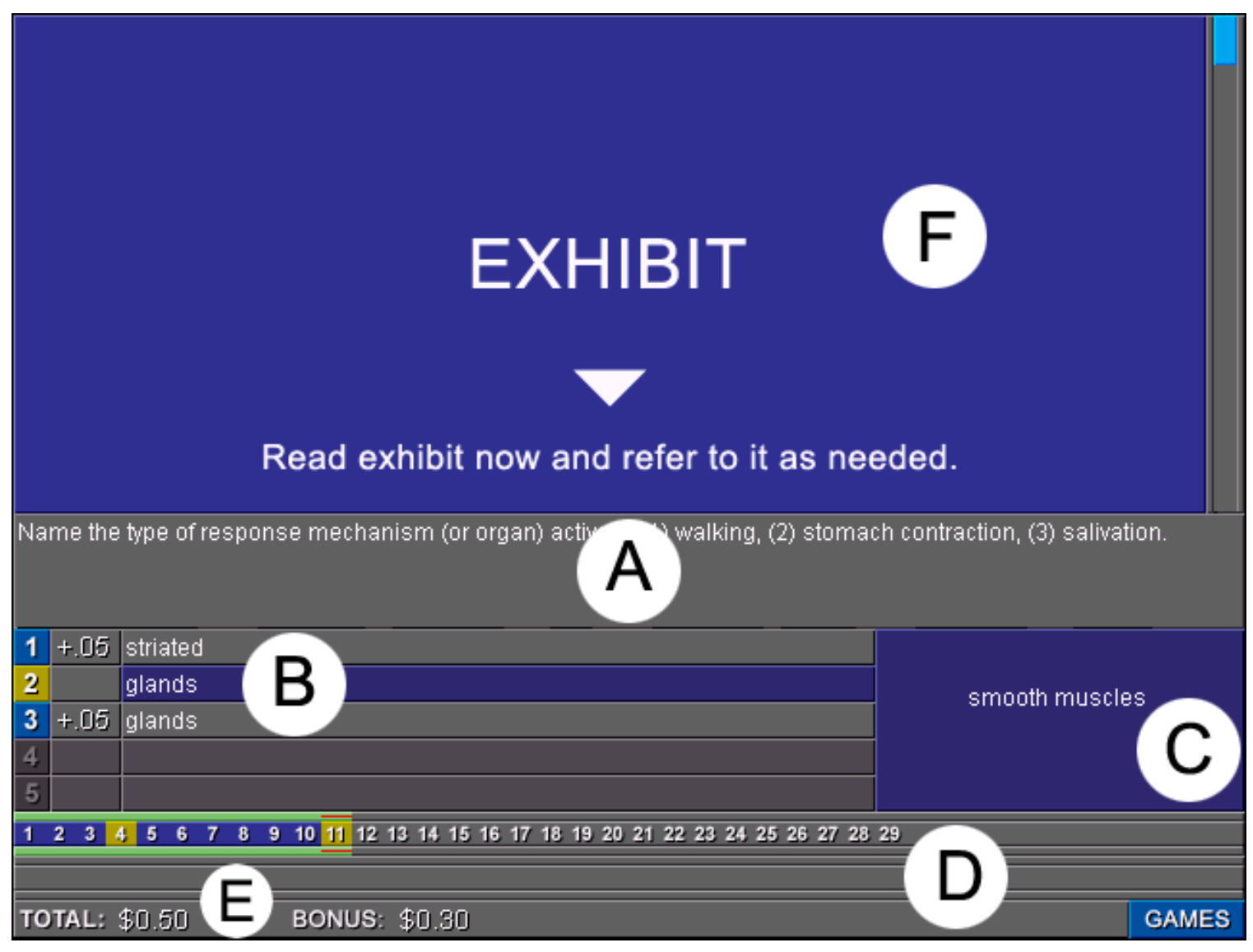

\section{Using the Program}

\section{Typing and Scoring Answers}

When you are using the program, sentences will appear in the center of your screen (in the area labeled " $\mathrm{A}$ " in the above figure). One or more of these sentences will be missing some text or will ask you a question. These sentences are called frames. Your task is to provide an answer or answers to each frame. Type your answers into the numbered blanks (position "B"). The TAB key, UP ARROW and DOWN arrow keys will move your cursor into a blank. (If the frame requires more than one answer, you may find it convenient to press the ENTER key on your 
keyboard to move the cursor to the next empty answer blank.) You may edit or delete your previously typed answers. When you are finished typing your answer(s), press the ENTER key on your keyboard. The computer will score your answers. Indicators located next to each of the answers will turn blue if your answer is correct. If your answer is not correct, the answer indicator will turn yellow. In the above figure, answers 1 and 3 were correct, and answer 2 was not.

Note the "+.07" next to the correct answers. This indicates that a total of ten cents were earned for this frame, as each correct response is worth seven cents (\$.07).

\section{Reviewing Your Answers}

After your answers have been evaluated, you will no longer be able to change them. Examine the correct answer by pressing the corresponding number key on your keyboard. In the above figure, answer 2 is incorrect and the user is holding down the number "2" on the keyboard to observe the correct response that is displayed in position " $\mathrm{C}$ " in the figure.

\section{Advancing to the Next Frame}

Use your SPACEBAR to advance to the next frame when you have finished with the frame.

\section{Frame Numbers}

A row of numbers is located across the bottom area of the screen (position " $\mathrm{D}$ " in the figure). Each of the numbers corresponds to the number of a frame. The number "1" corresponds to the first frame, the number "2" corresponds to the second frame, etc. The color of the frame number 
indicates its status. Red numbers indicate the frame that is currently active. Blue numbers indicate that the answers provided for that frame are correct. Yellow numbers indicate that at least one of the answers that for the frame is incorrect. In the above figure, the user provided incorrect answers for frames 4 and 11.

\section{Review of Frames}

Frames that were answered incorrectly (indicated by a yellow frame number) will be presented again after you have completed all of the frames. The session will end when all of your answers are correct.

\section{Phases}

Components are indicated by thin strips of color positioned directly above and below each frame number.

\section{Earnings}

You earn money when your answer is correct. The total amount of money that you earn in a session can be found next to the word "TOTAL:" at the bottom of your screen (position "E"). During each session you will also have the opportunity to add money to your bonus earnings. Your bonus earnings will be displayed next to the word "BONUS:". TOTAL earnings are paid to you at the end of each week, and BONUS earnings are paid to you upon completion of the experiment. 


\section{Exhibits}

Some sessions will require that you examine the contents of an exhibit to answer a frame (position "F"). These exhibits are found in this booklet and also within the computer program. When examining an exhibit within the computer program, you can click on the exhibit with your mouse to drag it up and down on the screen so that the relevant portions are visible.

Alternatively, you may use the scroller located on the top right-hand side of the screen to position the exhibit for viewing.

\section{Games}

Click on the button labeled "GAMES" located at the lower left corner of the program to open the games menu. When a game appears, you can start the game by clicking your left mouse-button. Press the DELETE button to exit the game and return to the program.

\section{Program Conventions}

The number of words needed to complete an answer are indicated by the number of blanks. Thus "__ " indicates a one-word response, whereas " " indicates a two-word response.

When asterisks ( $* * *)$ are used in place of blanks, fill in as many words as you think necessary to respond to the item. The abbreviation TT calls for a technical term. When it is used, a nontechnical word is incorrect. 


\section{Keyboard Functions}

GO TO NEXT ANSWER.

TAB

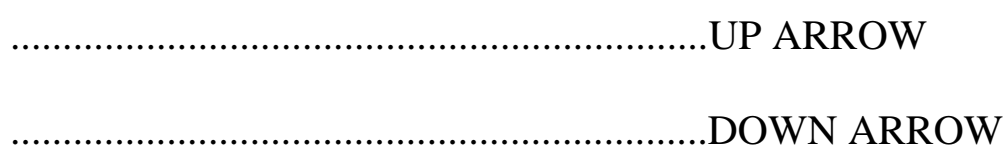

(ENTER)

EVALUATE ANSWERS

ENTER

DISPLAY CORRECT RESPONSE...............................................1, 2, 3, 4, 5

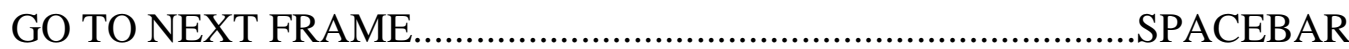

PLAY VIDEO GAME....................................................................... MOUSE BUTTON

RIGHT MOUSE BUTTON

EXIT VIDEO GAME.

DELETE

\section{Typing Conventions}

If the answer is:

Type the following:

$S^{\mathrm{D}}$

..sd

S

sdelta 